\title{
MATERNAL ACCULTURATION LEVELS AND THEIR EFFECT ON CHILDREN'S \\ FOOD INTAKE, MATERNAL PERCEPTIONS OF BODY SIZE AND \\ MATERNAL STRESS
}

\begin{abstract}
A Thesis
presented to

the Faculty of California Polytechnic University, San Luis Obispo
\end{abstract}

In Partial Fulfillment

of the Requirements for the Degree

Master of Science in Agriculture,

Food Science and Nutrition Specialization.

By

Tracie Chavoor

June 2013 
(C) 2013

Tracie Chavoor

ALL RIGHTS RESERVED 


\section{COMMITTEE MEMBERSHIP}

TITLE:

Maternal Acculturation Levels and their Effects on

Children's Food Intake, Maternal Perceptions of Body

Size and Maternal Stress.

AUTHOR:

Tracie Allison Chavoor

DATE SUBMITTED:

June 2013

COMMITTEE CHAIR:

Peggy Papathakis, Ph.D., RD Food Science and Nutrition professor

COMMITTEE MEMBER: $\quad$ Lisa Nicholson, Ph.D., RD Food Science and Nutrition professor

COMMITTEE MEMBER: $\quad$ Suzanne Phelan, Ph. D. Kinesiology professor 


\begin{abstract}
Maternal acculturation levels and their effect on children's food intake, maternal perceptions of body size and maternal stress.
\end{abstract}

Tracie Chavoor

In the U.S., obesity has hit alarming rates and affects Hispanic children disproportionately. Acculturation, the shift from one culture's values, beliefs and practices to those of a new culture, may have both negative and positive health effects on immigrants. The objectives of this study were to determine the association between the level of acculturation (low acculturation or high acculturation) of low-income Latina mothers and the foods consumed by their children ( 0 to 36 months old). The association between acculturation and maternal perceptions of infant and toddler body size, maternal perceptions of her own body size and maternal parenting stress were also investigated. This was accomplished in 3 stages. First, data was collected cross-sectionally from 68 participants of WIC. Mothers answered many questionnaires including Food Frequency Questionnaires for their children, body size perception scale questionnaires and a parenting stress test. General linear regression models were performed to investigate an association between acculturation and food frequency, maternal perceptions of body size and parenting stress. Each model controlled for child's age, maternal age, BMI (m/ $\left.\mathrm{kg}^{2}\right)$ and education level. Second, key informant interviews were conducted with nine public health professionals to help gain a better understanding of some of our findings and to develop questions for the third stage: focus groups. Third, a total of 32 mothers participated in focus groups to discuss their perceptions on acculturation and childfeeding habits, and children's body size. In addition, three mothers completed one-on-one 
interviews. From the cross-sectional analysis of the first study stage, the level of acculturation did not have a significant effect on any of the factors measured. For all mothers, however an increase in child's age was associated with an increase in both the amount of healthy foods and less healthy foods consumed; an increase in mother's BMI was associated with an increase in the children's consumption of less healthy foods (beta coefficient of $-0.042 ; p=0.035)$. An increase in child's age was associated with an increased likelihood of a mother to correctly estimate her own body size (beta coefficient of $0.041 ; p=0.043)$. An increase in maternal BMI was associated with an increased likelihood to underestimate her own weight as well as that of her child. During the second stage, a theme emerged from the focus groups that the mothers try to feed the children the way they were fed growing up but they encounter difficulties when they can't find the same ingredients and when their children start to prefer American foods. For all groups health was the number one factor they considered when choosing what to feed their children. The low acculturated mothers stated they wanted more education on how to assess if their child is at a healthy weight and admitted to not thinking about weight very much. In both groups, the mothers reported getting information about their child's weight from health care professionals but many mothers from the bi acculturated group mentioned not believing the doctor when they told them their child was overweight. Overall, the findings suggest that children's diets are not different by mother's acculturation level and that the children start to prefer American foods from a young age. Parental education for several areas were identified: 1) for mothers to learn how to continue with the healthful aspects of a traditional diet 2) teach parents about appropriate 
stages of growth for their children; 3) when trying to teach about food focus on other areas of health rather than weight since weight does not seem to resonate as a concern. 


\section{ACKNOWLEDGMENTS}

I would like to thank my graduate committee, who were so patient throughout the learning process and always took their time to answer my many questions. A special thank you to Dr. Papathakis who guided me through this entire process. I have grown so much under your guidance! Thank you to my awesome recruiter Gina- I could not have done the focus groups without you! And to all the WIC and Head Start employees who answered our many questions and allowed us to work in their centers- in particular Linda McClure, Suzanne Chasuk, Gary LiWang and Karen Easton. And last but not least, thank you to my wonderful husband who read all of my many manuscripts, listened to my presentations and continually supported me over the last 2 years. 


\section{TABLE OF CONTENTS}

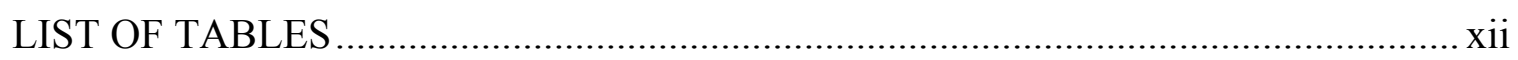

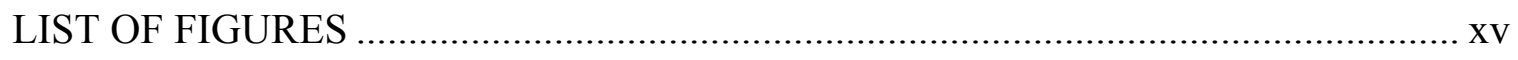

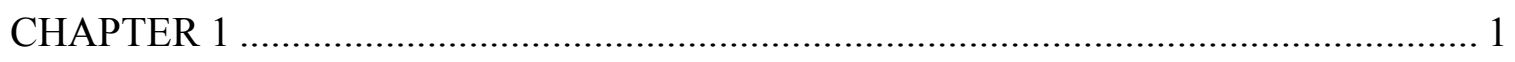

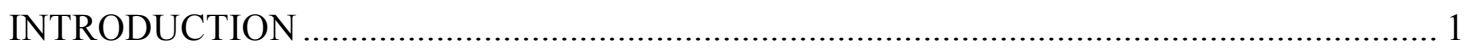

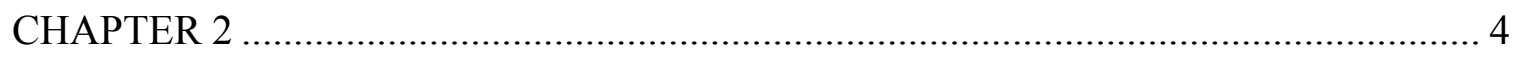

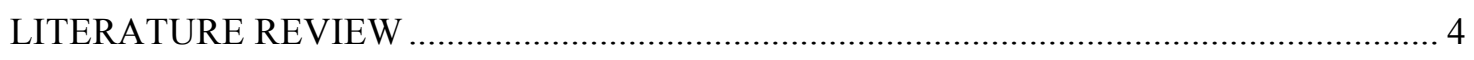

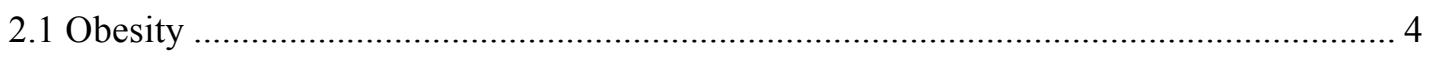

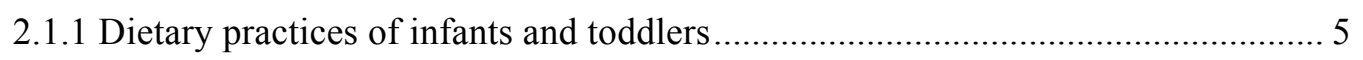

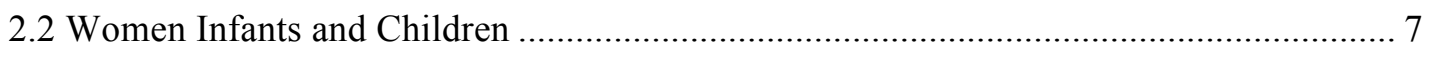

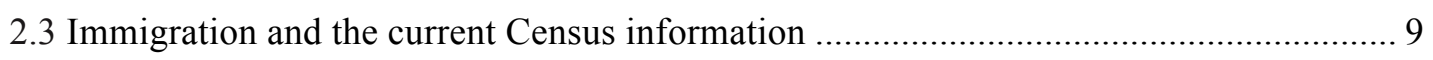

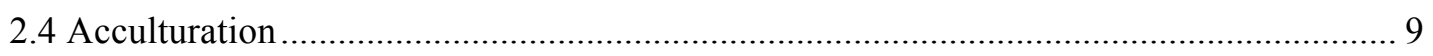

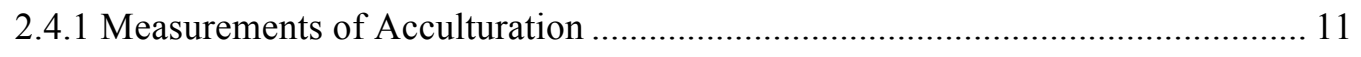

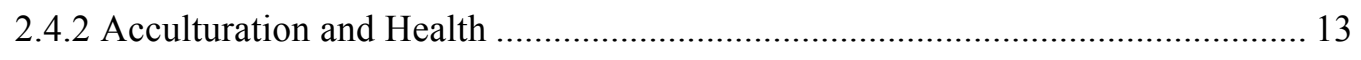

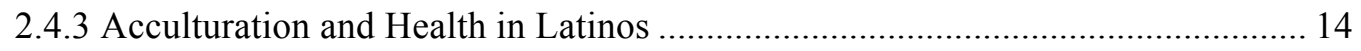

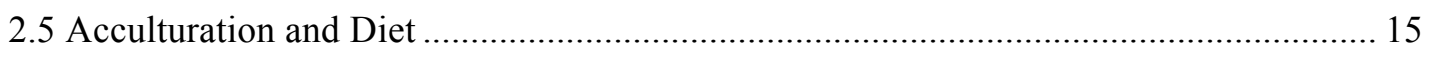

2.5.1 Dietary Acculturation in Mexican Americans ................................................. 18

2.5.2 Dietary Acculturation in Mexican American Children ..................................... 20

2.6 Acculturation and Body Image Perceptions ............................................................ 21

2.6.1 Maternal Perceptions of her Child's Body Weight ........................................... 21

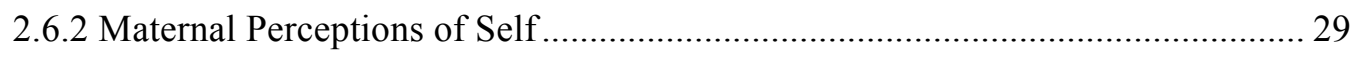

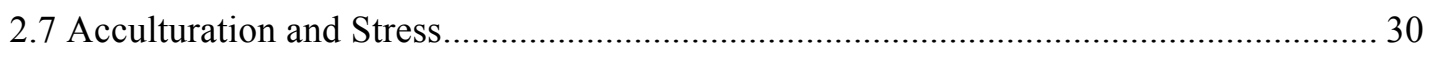

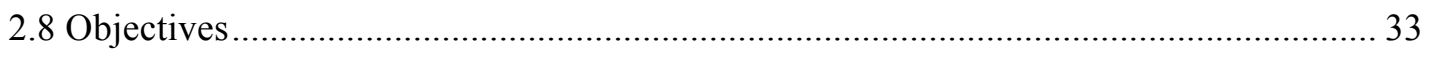


CHAPTER 3

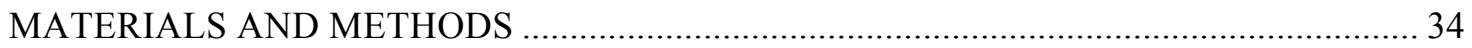

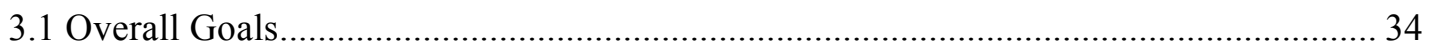

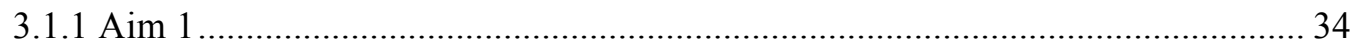

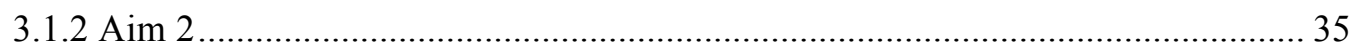

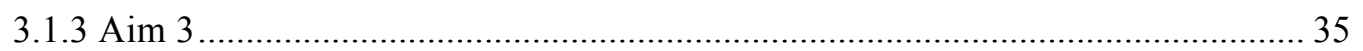

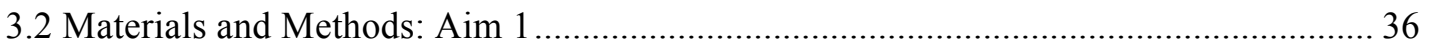

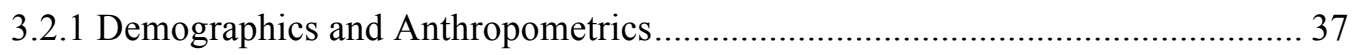

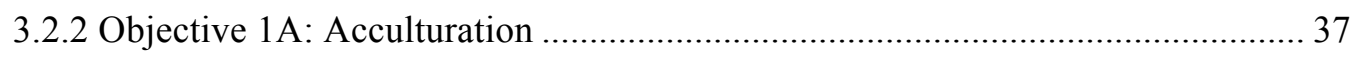

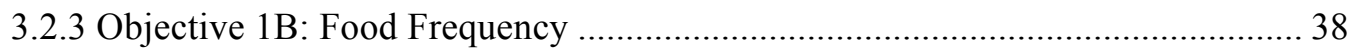

3.2.4 Objective 1C: Maternal Perceptions of Self................................................... 40

3.2.5 Part 2b: Maternal Perceptions of her Child ..................................................... 42

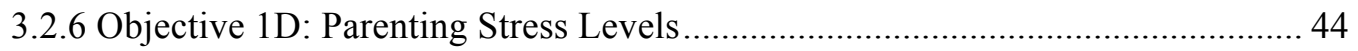

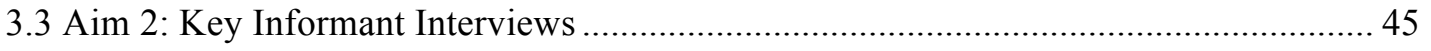

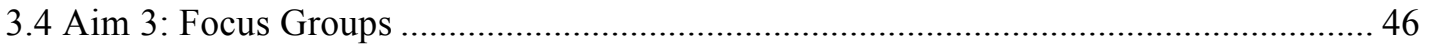

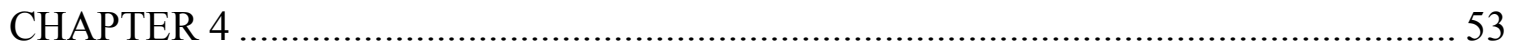

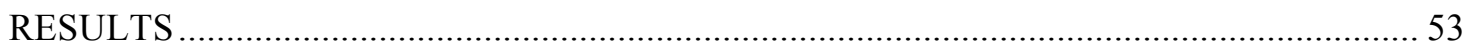

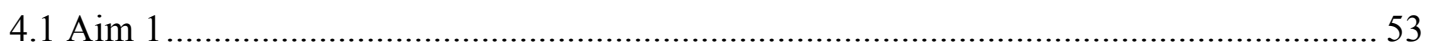

4.1.1 Objective 1A: Demographics and Anthropometrics ....................................... 53

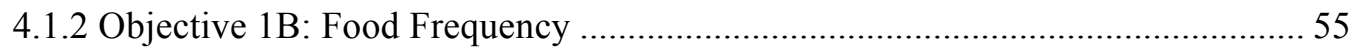

4.1.4 Objective 1Cb: Maternal Perceptions of Child's Body Size................................ 60

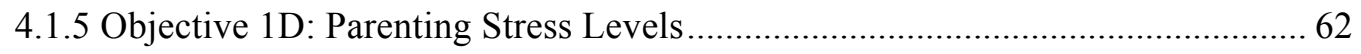

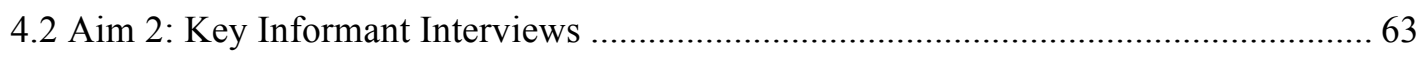

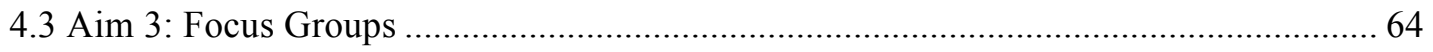

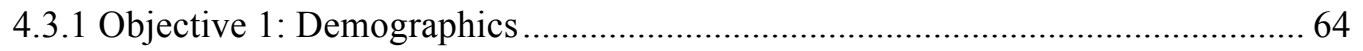




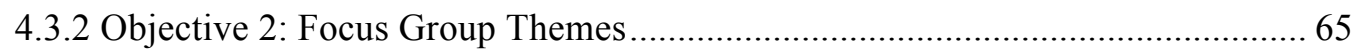

4.3.2 High acculturated focus groups/interviews: demographics .............................. 77

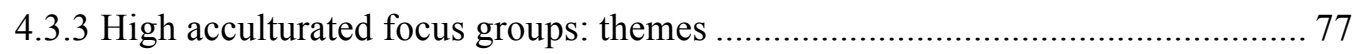

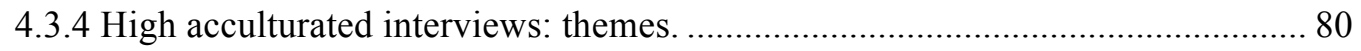

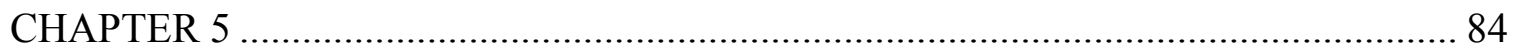

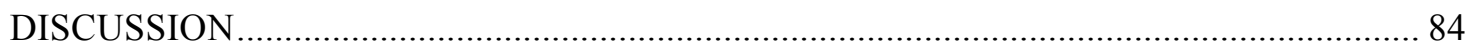

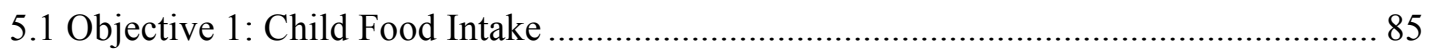

5.2 Objective 2: Maternal Perceptions of Body Size ..................................................... 90

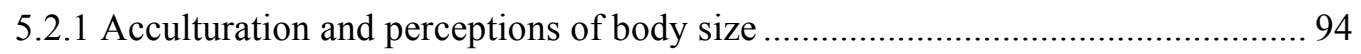

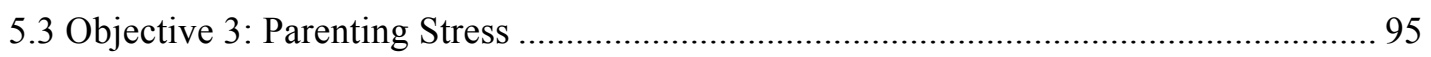

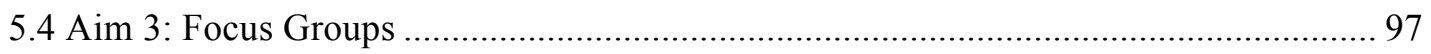

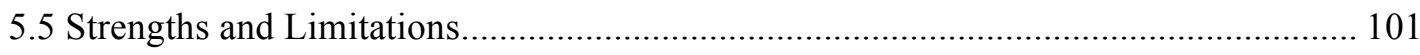

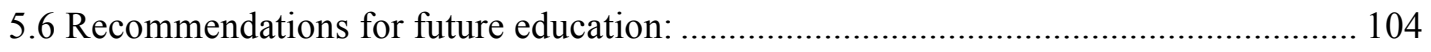

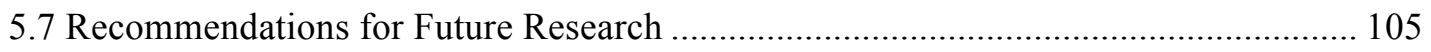

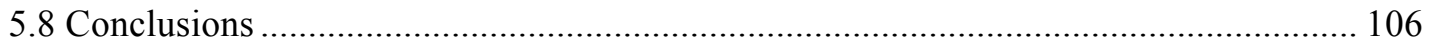

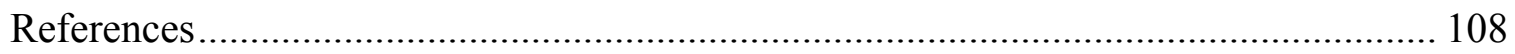

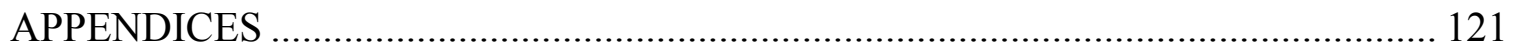

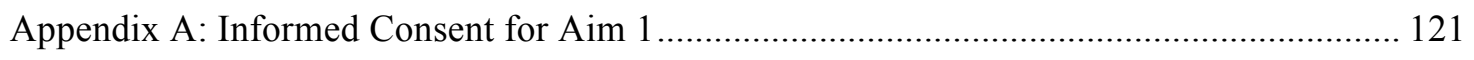

Appendix B: Food Frequency Questionnaires.............................................................. 124

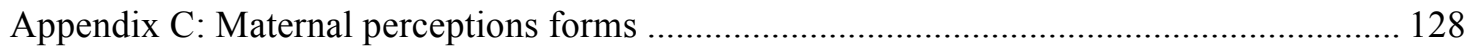

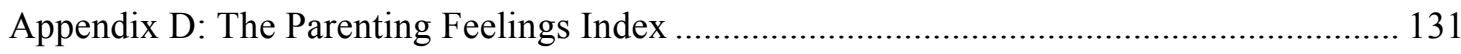

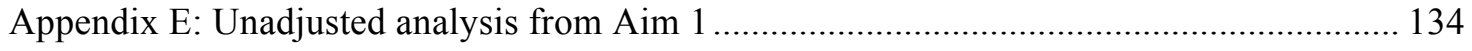

Appendix F: Informed consent for key informant interviews ......................................... 136

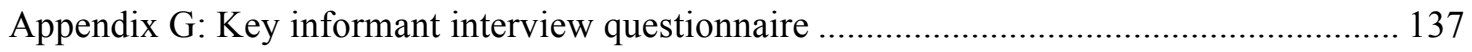

Appendix H: Full table of themes and quotes from Key Informant Interviews ..................... 139 
Appendix I: Research interest explanation form for focus groups

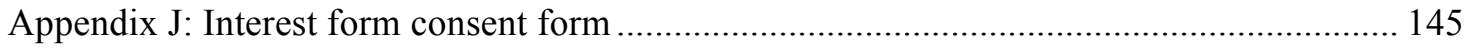

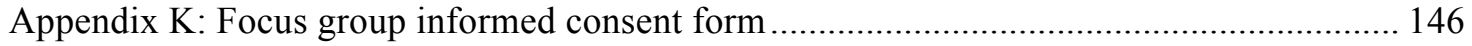

Appendix L: Bi-dimensional acculturation scale with demographics for focus group

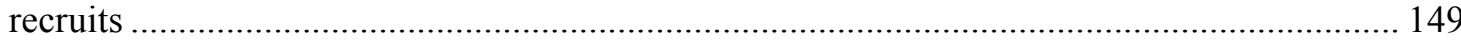

Appendix M: Demographics Questionnaire for focus group participants.............................. 155

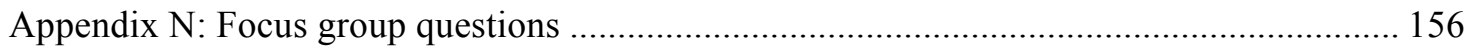

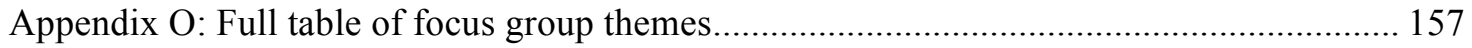




\section{LIST OF TABLES}

Table

Page

Table 3-1 Food items used to calculate the "health" foods composite score and the

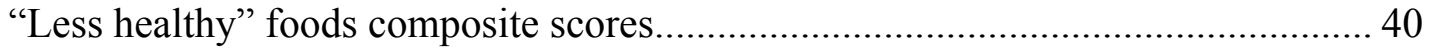

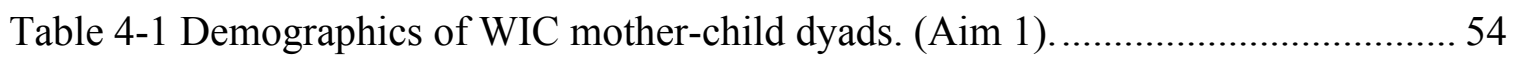

Table 4-2 Demographic factors by acculturation status (Aim 1)................................ 55

Table 4-3 Average weekly number of servings consumed of each of the food items used to make up our composite food scores for total participants, and comparing low acculturated and high acculturated participants. 56

Table 4-4 Unhealthy and healthy food's relationship by acculturation, child age, mother's age, mother's BMI and mother's education status, adjusted results. 57

Table 4-5 Maternal perceptions of women scores, an unadjusted analysis.

Table 4-6 Mother's actual versus perceived perception of her self and her child and it's relationship between acculturation, child age, mother's age, mother's BMI and mother's education status: adjusted.

Table 4-7 Maternal perceptions of infant scores aged 0-12 months old, an unadjusted analysis.

Table 4-8 Maternal perceptions of child scores aged 1-2 years old, an unadjusted analysis.

Table 4-9 Parenting stress score by subscale: parent related and child related stress and their relationship between acculturation, child age, mother's age, mother's BMI and mother's education status: adjusted. 
Table 4-10 Focus group demographics in focus group participants and those who dropped out. Aim 3

Table 4-11 Focus group themes separated by level of acculturation: differences between how the mothers were fed and how they now feed their children. 69

Table 4-12 Focus group themes separated by level of acculturation: how more or less Americanized mothers differ in how they feed their children. 70

Table 4-13 Focus group themes separated by level of acculturation: what is most important in how they choose foods for their children. 70

Table 4-14 Focus group themes separated by level of acculturation: differences between more and less Americanized mothers in how they choose foods for their children. 71

Table 4-15 Focus group themes separated by level of acculturation: how a child's diet affects his or her weight.

Table 4-16 Focus group themes separated by level of acculturation: how the mothers decide if their child is at a good weight and the difference in this between levels of acculturation.

Table 4-17 Focus group themes separated by level of acculturation: how the mother's view of their child's weight effects what they feed them. 73

Table 4-18 Focus group themes separated by level of acculturation: how to address overweight and underweight infants. 74

Table 4-19 Focus group themes separated by level of acculturation: how to address overweight and underweight toddlers. 75 
Table 4-20 Focus group themes separated by level of acculturation: Selectivity in feeding children and how acculturation affects selectivity...................................... 76

Table 4-21 High acculturated focus group and interview demographics, the difference between recruited mothers between mothers who participated and drop outs.

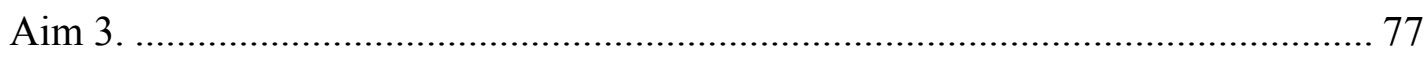

Table 4-22 Themes from high acculturated focus group ............................................... 79

Table 4-23 Themes from 3 interviews with high-acculturated mothers.......................... 81

Table 5-1 Maternal perception of infant scores aged 0-12 months old. ........................... 92 


\section{LIST OF FIGURES}

Figure Page

Figure 1-1 Proportion of U.S children (2-5 years old) with obesity (defined as having

a BMI the $\geq 95^{\text {th }}$ percentile) by ethnicity ..................................................... 5

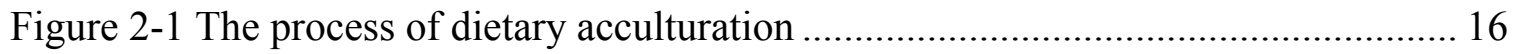

Figure 2-2. The African-American Infant Body Habitus Scale (AAIBHS) .................... 23

Figure 3-1 A silhouette scale developed by (Stunkard et al.) used to assess maternal perceptions of body size. Below each score are the correlating BMIs .................. 41

Figure 3-2 Infant figures adapted from Boyington et. al. Silhouette scale used to assess maternal perceptions of infant's body size (age 12 months and under) 43

Figure 3-3 Toddler figure scale developed by Erica Melling..... 44 


\section{CHAPTER 1 \\ INTRODUCTION}

Obesity is America's latest epidemic. From 2007 to 2008 obesity affected 33.8\% of Americans with $32.3 \%$ of men and $35.5 \%$ of women (Flegal, 2010). Adults are considered overweight if they have a body mass index (BMI) from 25.0 to 29.9 and obese if they have a BMI of 30 or greater (CDC, 2011a) Obesity not only impacts adults, but children as well. An estimated 2.7 million children in the U.S. have a BMI $\geq$ the $99^{\text {th }}$ percentile (Skelton, Cook, Auinger, Klein, \& Barlow, 2009). A BMI $\geq$ the 99th percentile in children has been associated with increased waist circumference, insulin resistance, blood pressure and lower high density lipoprotein (HDL) cholesterol. A third of children who have a BMI $\geq$ the 99th percentile have metabolic syndrome (Skelton et al., 2009), which is a group of symptoms which increase risk of cardiovascular disease and diabetes (NIH, 2011).

Mexican Americans children are especially affected by obesity. Nearly $19 \%$ of Mexican American children at 3 years of age had a BMI $\geq 95 \%$ as opposed to $8.5 \%$ of White and $12.4 \%$ African American children in the most recent NHANES data (Taveras, 2010). This is especially disconcerting because Mexican Americans have some of the highest rates of type 2 Diabetes Mellitus (DM) in the U.S. (Cossrow, 2004). That increased health risk, along with the increasing number of Mexican Americans in the U.S. means this is an important population to focus obesity prevention efforts.

In $201012.9 \%$ of the U.S. population was foreign born and $29.3 \%$ of those originated from Mexico (Bureau, 2010). When someone immigrates into a new country, they begin to undergo a process called acculturation. This is a dynamic process in which 
a person or group from one culture is introduced to a new culture and begins to adopt certain aspects of that culture, including their "behaviors, attitudes, norms and values" (Liu, Chu, Frongillo, \& Probst, 2012; Teske \& Nelson, 1974). Acculturation can cause a change in diet called dietary acculturation. This can lead to both harmful and healthful food practices. For instance, Hispanics who immigrate into the U.S. may start consuming foods higher in sugar (more desserts, sodas) but they also may eat fewer foods containing lard (less flour tortillas and refried beans) (Satia-About, Patterson, Neuhouser, \& Elder, 2002). While there has been a lot of research on the impact of acculturation on the eating habits of adults (G. X. Ayala, Baquero, \& Klinger, 2008; Duffey, Gordon-larsen, Ayala, \& Popkin, 2008; Montez \& Eschbach, 2008; Pérez-Escamilla, 2009), there has been little research focusing on how a mother's level of acculturation effects what she feeds to her children.

Perceptions of an ideal weight are often times culturally determined and can also be affected by acculturation. Some studies show that with an increase in acculturation, Mexican Americans begin to prefer a slimmer figure (Guendelman, Fernald, Neufeld, \& Fuentes-Afflick, 2010; Olvera, Suminski, \& Power, 2005). This is important to note because a parent's perception of their child's weight can affect how they feed them (Boyington \& Johnson, 2004). There has been little research on the effect of acculturation on maternal perceptions of body sizes of herself and of infants and children.

There is some evidence that the Mexican culture has a protective effect on mental health, including stress management (Acevedo, 2000; Campos, Schetter, Walsh, \& Schenker, 2007; Nancy Landale, Oropesa, Llanes, \& Gorman, 1999). Interestingly, one study found that unmarried Mexican-American women who only spoke Spanish had 
higher mental health scores than English speaking women indicating that the lower acculturated women were mentally healthier than the more acculturated women. This relationship did not remain for married women (Acevedo, 2000). There is little research available on the effect of acculturation on parenting stress.

This research explores the relationship between acculturation and diet, maternal perceptions of body size and parenting stress in low income Hispanic mothers and their children living in the U.S.. 


\section{CHAPTER 2}

\section{LITERATURE REVIEW}

\subsection{Obesity}

Approximately 18\% of children were considered obese in 2010 (CDC, 2013).

Overweight and obesity in children are determined by interpreting BMI percentiles. A child is considered overweight if their BMI is between the $85^{\text {th }}$ and $95^{\text {th }}$ percentiles and obese if their BMI is at or above the $95^{\text {th }}$ percentile (CDC, 2011b). The prevalence of obesity in young children continues to increase. The prevalence of childhood obesity went from 7\% in 1980 to almost 18\% in 2010 (CDC, 2013) From 1999-2004 the estimated number of children in the U.S. with a BMI $\geq$ to the $99^{\text {th }}$ percentile was 2.7 million (Skelton et al., 2009). Obesity rates are also increasing with age. A study conducted in New York followed 1,713 children from 1 year until 5 years of age. Rates of obesity increased from $7.5 \%$ at one year of age to $29.8 \%$ by 5 years of age (Irigoyen, Glassman, Chen, \& Findley, 2008). These findings suggest that early childhood is an important time for obesity prevention, especially when considering that up to $80 \%$ of obese children will become obese adults (Cali \& Caprio, 2008).

Among women, ethnicity has a significant role in obesity. Mexican American women between 20 and 39 years of age have some of the highest rates of obesity in the U.S. (38.4\%), second only to African American women with rates of 49.0 and white women (30.7\%) (Hedley et al., 2004). Similar trends are seen in children as shown in Figure 1.1. In Mexico $71.9 \%$ of women and $66.7 \%$ of women are overweight or obese. About $22 \%$ of Mexican adults were obese in 1993 which increased to 30\% by 2006 (Olaiz et al., 2006). In Mexico, the rates of obesity for children are lower than in the U.S. In children 
under 5 years the prevalence of obesity in 2012 was 9.8\% (Encuesta Nacional de Salud y Nutricion de Mexico, 2012). The most recent NHANES data identified 26\% of Mexican American children between the ages of 2 and 5 years were overweight or at risk or becoming overweight (Taveras, Gillman, Kleinman, Rich-Edwards, \& Rifas-Shiman, 2010).

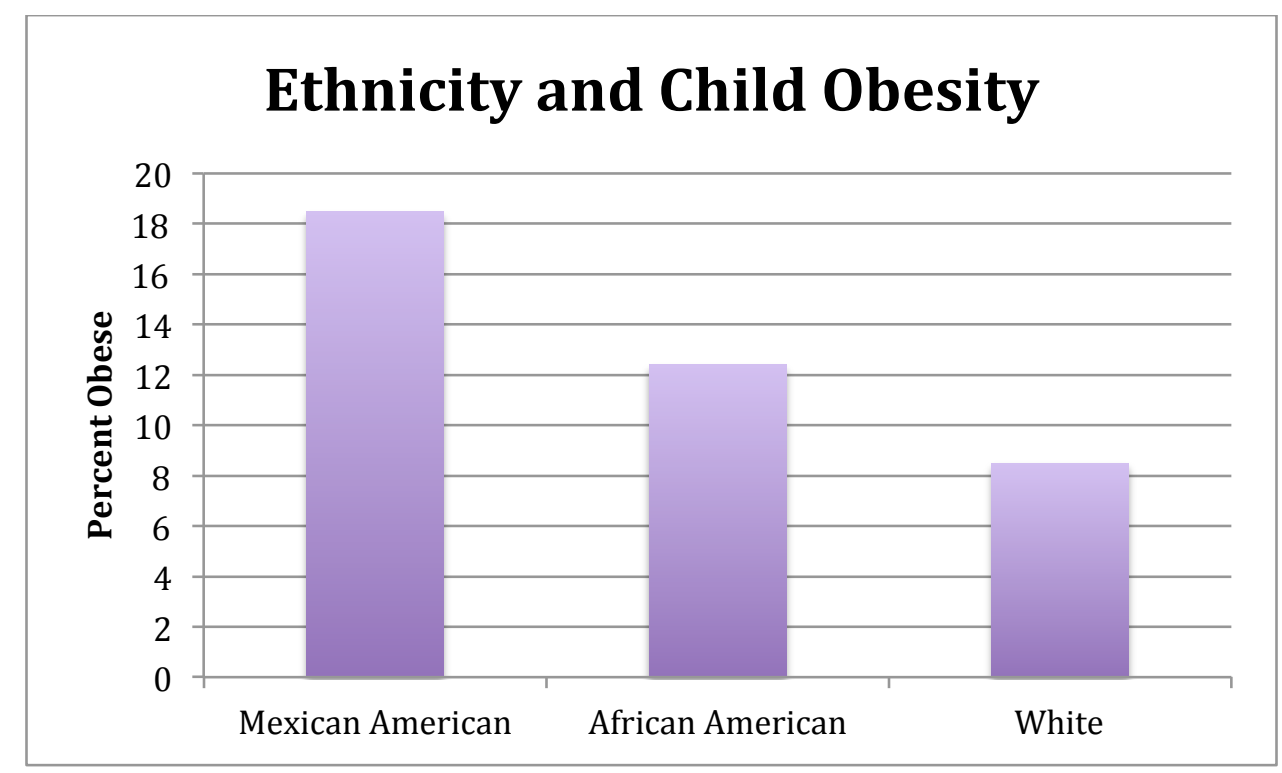

Figure 1-1 Proportion of U.S children (2-5 years old) with obesity (defined as having a BMI the $\geq 95^{\text {th }}$ percentile) by ethnicity. Adapted from (Taveras et al., 2010).

\subsubsection{Dietary practices of infants and toddlers}

In a sample of 3,022 infants and toddlers between 4 and 24 months of age the Feeding Infants and Toddler study assessed dietary intake and compared it between Hispanic $(\mathrm{n}=371)$ and non Hispanic children $(\mathrm{n}=2,637)$ (Briefel, Ziegler, Novak, \& Ponza, 2006). In general, infants and toddlers are taking in more energy than they need. For the non-Hispanic infants (6-11 months old) energy consumption was $22 \%$ higher than the estimated energy requirements, and for toddlers, mean energy intake was $31 \%$ above the estimated energy requirements. Typical intake of fat for non-Hispanic toddlers was 
$33.2 \%$ of their total caloric intake, with carbohydrates at $53 \%$ and protein at $15 \%$ of total energy (Briefel et al., 2006). The non-Hispanic infants and toddlers, in general, consumed adequate vitamin and mineral intakes however most toddlers took in an average of $8 \mathrm{~g}$ of fiber per day which is much lower than the recommended $17 \mathrm{~g}$ (Briefel et al., 2006).

Hispanic infants and toddlers had very similar nutrient intake as the non-Hispanic, however a few of the categories differed. Hispanic infants and toddlers, on average, consumed $17 \%$ and $31 \%$ more energy respectively than the estimated daily requirements, which was not significantly different than the non-Hispanic children (Briefel et al., 2006). Their fat intake was no different than the non-Hispanic children, but their energy intake from carbohydrates was significantly more than non-Hispanic children $(55.6 \%$ versus $53.0 \% \mathrm{p}$-value $<0.01)$. These differences are not of concern, however, because the dietary guidelines recommend consumption of carbohydrates to be anywhere from 45 to $65 \%$ of total calories (U.S. Department of Agriculture \& U.S. Department of Health and Human Services, 2010). The proportion of Hispanic toddlers who had an intake of fat above the acceptable range was significantly greater than the non-Hispanic toddlers ( $46 \%$ versus $36 \%$ ). Hispanic toddlers consumed $9 \mathrm{~g}$ of fiber a day on average which was slightly more than the average non-Hispanic intake but still below the recommended daily intake of $17 \mathrm{~g}$ (Briefel et al., 2006).

In general, infants and toddlers living in the U.S. are taking in between 17 and $33 \%$ more energy than they need primarily in the form of excess fat and carbohydrate (Briefel et al., 2006). All young children appear to be getting a sufficient amount of micronutrients but their diets are lacking in fiber (Briefel et al., 2006). 


\subsection{Women Infants and Children}

The Special Supplemental Nutrition Program for Women, Infants and Children (WIC) is a federal grant program administered by the Food and Nutrition Services. It targets pregnant and breastfeeding women and children ages five years and under (“About WIC," 2012). One of the criteria for participation includes people with an income less than $185 \%$ of the federal poverty level ("WIC eligibility requirements," 2012). For example, in San Luis Obispo (SLO) County, a three person family must be making less than or equal to $\$ 36,131$ annually to qualify for WIC (California WIC, 2013). WIC considers mothers enrolled in Cal Fresh, (a government program which gives food assistance to the underprivileged) or Medicaid, (a federal health benefit program for people of low income) as automatically eligible for WIC. Also, those who are considered as nutritionally at risk by a health professional are considered eligible ("WIC eligibility requirements," 2012). Eligible participants receive vouchers for supplemental foods such as milk, cheese, whole grains, breakfast cereal, sources of protein including beans, peanut butter, soy and tofu and a variety of fruits and vegetables (The California WIC Program, 2011). Participants also receive breastfeeding counseling with a lactation consultant, nutrition education, farmers market vouchers, screening and referrals for other health related issues (“About WIC,” 2012). Nutrition education includes information on prenatal nutrition, breastfeeding, healthy foods for children and parenting advice (The California WIC Program, 2012).

California has the largest WIC program in the nation serving an average of $1,466,564$ women, infants and children monthly ( $16.4 \%$ of all national WIC participants) (California WIC, 2012; National WIC Association, 2013). California WIC participants 
are composed of $58 \%$ children 1 to 5 years, $20 \%$ infants $0-11$ months and $22 \%$ women either prenatal, postpartum or breastfeeding (California WIC, 2012). In 2011, an average of 841,137 children and 294,283 infants received WIC benefits each month (National WIC Association, 2013). There were approximately 2,496,015 children 5 years and under residing in California in 2011, which means that approximately $45 \%$ of children 5 years and under residing in California were enrolled in WIC (United States Census Bureau, 2013). Around $42 \%$ of U.S. WIC participants are Hispanic, but in California the Hispanic WIC population is much higher at $63.9 \%$. SLO County's Hispanic WIC population is representative of the state in that $62.9 \%$ of participants are Hispanic with $39 \%$ of all participants originating from Mexico (Papathakis, Nazmi, Phelan, \& Engle, 2013).

Approximately $18.9 \%$ of Hispanic WIC infants at the age of 1 year are considered obese and $17 \%$ of Hispanic WIC children who are 2 years or older are obese and (USDA Food and Nutrition Services, 2010). In 2010 about 25\% of all WIC participants were labeled as high weight for height and around 70\% of WIC mothers were overweight (USDA Food and Nutrition Services, 2010). In the past, WIC has been shown to have a significant impact on the diet of participants. Pregnant women involved in WIC have been shown to consume greater amount of most micronutrients; infants consume more iron and vitamin $\mathrm{C}$ and children consume more iron, vitamin $\mathrm{C}$, vitamin $\mathrm{B}-6$, thiamin and niacin compared with non WIC participants (Rush, 1986). A study conducted with 499 preschool children found that the children involved in WIC had a significantly higher intake of zinc and iron than the non-WIC group (Rose, Habicht, \& Devaney, 1998). Arica et al. conducted research in 4,219 women enrolled in WIC and 785 non-WIC women and found that WIC women were consuming more nutrient dense foods (the foods supported 
by WIC coupons). They also found that WIC participants spent less money on foods eaten out (Arcia, Crouch, \& Kulka, 1990). With the large population that WIC serves, and its many dietary benefits, WIC has the opportunity to positively affect the diet of many women, infants and children.

\subsection{Immigration and the current Census information}

Of the US population, $16.3 \%$ is of Latino descent and $10 \%$ of the total population is Mexican Latino, according to the 2010 US Census Bureau. The Mexican population in the U.S. grew by 54\% between 2000 and 2010 (Ennis, Rios-Vargas, \& Albert, 2011). In $2011,38.1 \%$ of California's population was of Hispanic or Latino descent and approximately $28.6 \%$ of the California population speaks primarily Spanish (United States Census Bureau, 2013). In 2010, 12.9\% of the U.S. population was foreign born and 29.3\% of those originated from Mexico (Grieco et al., 2012). Being foreign-born has both positive and negative aspects seen in the research. A positive aspect of being foreign born includes a higher likelihood of being married and living with family, especially within multigenerational families. Foreign born people are however more likely to be living in poverty, are less likely to be high school graduates and are more likely to be in the labor force than native-born citizens (Grieco et al., 2012). The Mexican-American segment of the population is increasing and may be at higher risk for certain diseases, therefore it is important for health care professionals to understand the culture to better support their physical, mental and social well-being and to prevent diseases.

\subsection{Acculturation}

A person from one country undergoes acculturation when introduced to a new country and he or she begins to adopt certain aspects of the new country's culture, 
including their "behaviors, attitudes, norms and values" (Pérez-Escamilla \& Putnik, 2007; Satia-About et al., 2002; Teske \& Nelson, 1974).

Acculturation is very complex. It affects many life factors, is nonlinear in time, can affect both cultures involved and can impact people at both the individual and group level (Satia-About et al., 2002). In the past experts believed that acculturation was a series of stages in which one phase must occur before the next can occur (Satia-About et al., 2002). This view has changed and many now see acculturation as a multidimensional process. There is no order in which people take on new dimensions of the host culture. People can take on a part of the new culture only to reject it again later (Satia-About et al., 2002).

Acculturation is bidirectional, not only on the time spectrum, but also in the way it can affect both cultures involved (Teske \& Nelson, 1974). When a group of Latinos immigrate to a city in the U.S. they may begin to adopt the U.S. culture, such as food, faith, and values, but it is very likely that the U.S. city will begin to embrace some of the Latino culture as well (Teske \& Nelson, 1974). An example of this is the wide availability of Mexican restaurants in Southern California where many Latinos have settled. The main direction of acculturation is influenced by the level of dominance one culture has over the other. A dominant culture is the one that holds power, excludes the minority from certain activities and makes it difficult for the minority to hold positions of power (Teske \& Nelson, 1974). The host culture is not always the dominant one, sometimes a more dominant culture will immigrate into a host culture. South Africa is a clear instance of foreigners, in this case the Dutch and English, becoming the dominant 
power over the host, in this case native South Africans, thereby influencing the culture they arrive into (Jones, 2010).

Acculturation can occur at both an individual and group level. An individual will undergo acculturation but if the individual is within a group of the same culture, the group's acculturation process will have an affect on the individual (Teske \& Nelson, 1974). People within a group may experience the acculturation process differently. According to Perez-Escamilla, there are four different acculturation trajectories a person may take (2007). First, an immigrant may become fully acculturated, welcomed into the host culture and reject their own culture. Second, they may reject the host culture, refusing to acculturate at all. Third, they may accept certain aspects of the new culture but refuse others, or lastly, they may lose their own culture without being integrated into the new culture and become marginalized (Pérez-Escamilla \& Putnik, 2007).

\subsubsection{Measurements of Acculturation}

Since acculturation is multifaceted, bi-directional, has no set pattern and can occur at many levels, it is very difficult to measure accurately. To determine acculturation level for research, single item measures of acculturation scales can be used. Single-item measures include language proficiency, self-identification, residency, generation level, birthplace and friendship preferences (Chaparro, Langellier, Kim, \& Whaley, 2011; Lind et al., 2012; Liu et al., 2012; Satia-About et al., 2002). Single-item measurements or a combination of single-item measurements are very simple and easy to perform. Single-item measurements are an accepted way to measure acculturation and are often used in public health settings (Chaparro et al., 2011; Lind et al., 2012; Liu et al., 2012; Satia-About et al., 2002). That being said, because of acculturation's broad nature, 
single item measurements aren't the preferred way to assess acculturation. Language use does encompass one side of adapting to a culture but it ignores the adaptation of values. Country of origin, as a single item, doesn't change and therefore overlooks the fact that acculturation is a process (Coronado, Thompson, Mclerran, Schwartz, \& Koepsell, 2005).

Acculturation scales are considered more accurate due to their consideration of the multiple spheres of acculturation. They can include questions about language, media preferences, friend preferences and questions of self-identity. Although more accurate, they are lengthier than single item measures and can therefore be impractical in some studies (Satia-About et al., 2002). Two acculturation scales are typically used with Hispanics. One is the Acculturation Rating Scale for Mexican Americans (ARSMA) and the other is the Short Acculturation Scale for Hispanics (Satia-About et al., 2002). The measurement a study uses depends on resources and needs. Each measurement has benefits and limitations.

The ARSMA contains 20 questions including the following topics: language use, ethnic identification, cultural heritage, ethnic behavior and ethnic interaction. This scale categorizes people into 5 different classifications: "very Mexican, Mexican-oriented bicultural, 'true' bicultural, Anglo-oriented biculturals and very Anglicized" (Coronado et al., 2005). The Short Acculturation Scale for Hispanics was adapted from the ARSMA and only includes two measurements of the ARSMA including language familiarity and use and ethnic interaction. It contains 8 questions in total. This scale categorizes people into numbers: 1 to 4 . A score of 1 being the lowest level of acculturation and 4 being the highest (Coronado et al., 2005). The Short Acculturation Scale is quicker to perform. 
Both scales allow for various levels of acculturation and take into account the multifaceted nature of acculturation, however dividing a sample into 4 or 5 different categories can be an issue with smaller sample sizes. The Bi dimensional acculturation scale for Hispanics (BAS) is another scale that asks questions on language use, language proficiency, electronic media use and celebrations. This scale puts people into three categories: high adherence to the non-Hispanic domain, biculturalism and high adherence to the Hispanic domain. It asks 21 questions in total, each having a four-point Likert scale. This scale takes longer to conduct than the short ARSMA but the smaller number of categories is better for a smaller sample size (Marin \& Gamba, 1996). The BAS was tested and found valid among both Central Americans and Mexican Americans (Marin \& Gamba, 1996).

\subsubsection{Acculturation and Health}

Acculturative changes often affect one's health, making acculturation an important area of study in public health. Foreign-born Latina mothers have better birth outcomes than U.S. native-born mothers. In a study of 22,892 infants, immigrants from Mexico and other Latino countries had smaller rates of low birth rates (3 and 7\%, respectively) than from mothers of Mexican and other Hispanic descent who were born in the U.S. (14 and 15\% respectively) (J. Collins \& Shay, 1994). Immigrants from Puerto Rico to the U.S. have lower rates of infant mortality in their offspring than do Puerto Rican women born on the mainland, although reasons for this are complex (N Landale, Oropesa, \& Gorman, 2000). Another study of immigrants from Poland, Turkey and Iran living in Sweden showed that one of the links between self reported poor health and ethnicity was poor acculturation. This suggests that an increase in acculturation into the 
Swedish culture would improve their health (Wiking, Johansson, \& Sundquist, 2004). A study of Chinese, Korean and Vietnamese adults in the U.S. showed an increase in acculturation led to an increase in BMI, especially among men. They theorized that men acculturate more quickly than women because they are more likely to be out of home and in the workplace (L. Chen, Juon, \& Lee, 2012).

\subsubsection{Acculturation and Health in Latinos}

Acculturation has many specific health effects on the Latino population in the U.S.. A Mexican immigrant is more likely to be a heavy smoker the longer he/she has lived in the U.S. (Lopez-Gonzalez, Aravena, Hummer, \& Lopez-gonzalez, 2005). An increase in acculturation has also been associated with an increase in consumption of alcohol in Latinos (Lopez-Gonzalez et al., 2005). Birth outcomes for Mexican-origin mothers specifically residing in the U.S. are equally as favorable or possibly more favorable as native white women, even though the Mexican-origin mothers tend to have a much lower socioeconomic status (J. Collins \& Shay, 1994; N Landale et al., 2000). Another study showed that less acculturated Hispanics had lower rates of low birth weight than more acculturated women residing in the U.S. (Scribner \& Dwyer, 1989). These findings suggests that the Mexican culture has a protective effect on birth outcomes and that this can be lost as they acculturate/assimilate into the U.S. culture (N Landale et al., 2000). Some of the suggested cultural influences on birth outcomes include: better nutrition, less use of tobacco and alcohol, fewer premarital births and more respect for parental roles (Scribner \& Dwyer, 1989). Despite the high prevalence of obesity in Mexico, many studies suggest that an increase in acculturation can lead to an increase in BMI among Latinos (Fuentes-afflick \& Hessol, 2008; Liu et al., 2012; Van 
Hook, Baker, Altman, \& Frisco, 2012). A variety of lifestyle changes could cause an increase in BMI however the two main contributors are more sedentary behaviors and dietary acculturation.

\subsection{Acculturation and Diet}

Dietary acculturation is a process in which immigrants start to adopt the dietary practices of the host culture. This complex process is described in Figure 2-1. Dietary acculturation is different than a nutrition transition, when an entire country will shift their diet such as the United States in increasing the energy dense diets in the last 20 years (Satia, 2010). Like acculturation, dietary acculturation is multidimensional, multifactorial and different for each individual. It is multidimensional in that a person may choose to adapt some new dietary practices and reject others or one may keep some of their own practices while changing others. Dietary acculturation can lead to both harmful and healthful food practices. For instance, Hispanics in the U.S. may start consuming foods higher in sugar (more desserts, more sodas) but they also may eat fewer foods containing lard (Satia, 2010). 


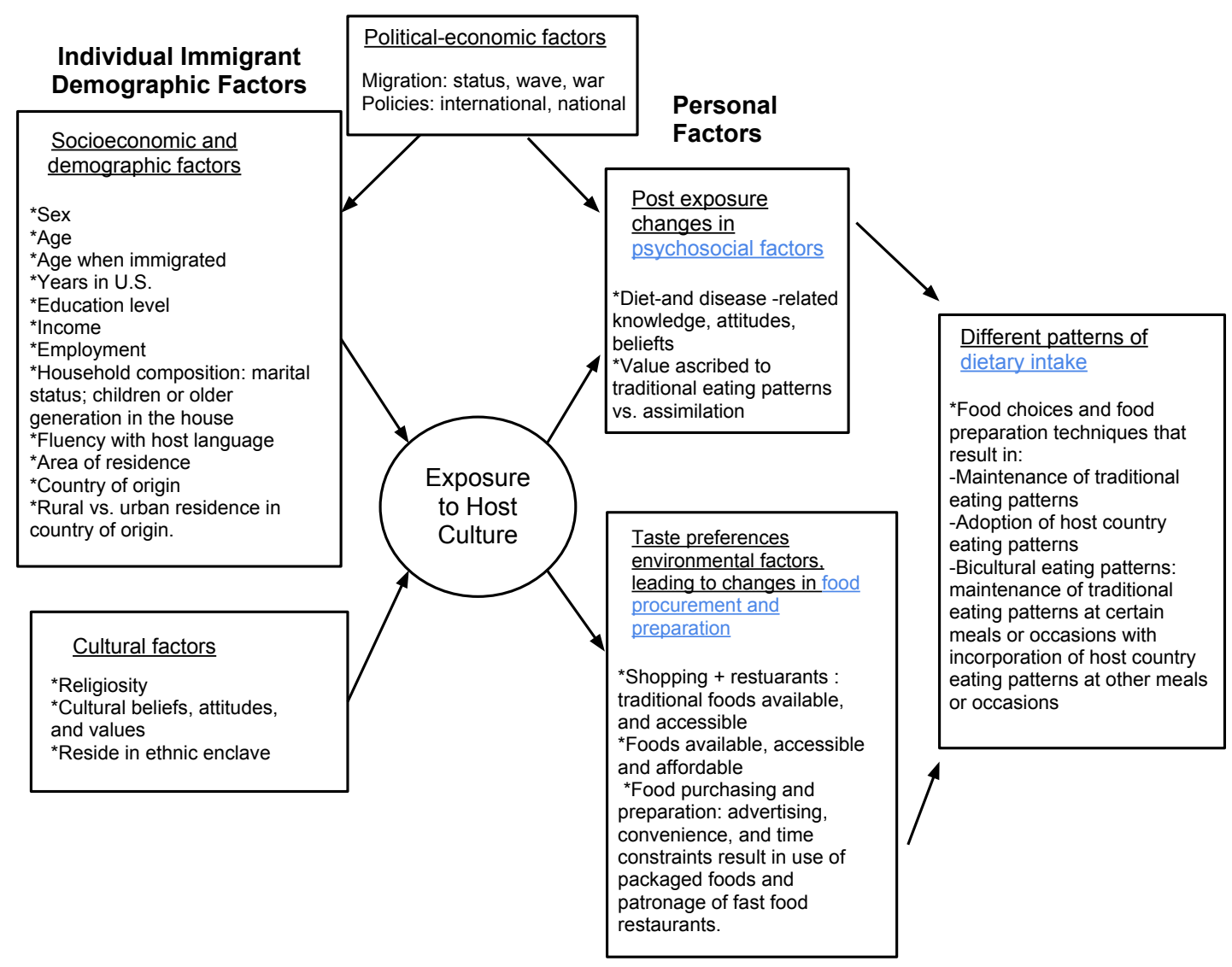

Figure 2-1 The process of dietary acculturation. Adapted from (Satia, 2010).

Dietary acculturation is multi-factorial in that diverse factors affect what foods someone will accept and reject, and how quickly they will acculturate. Some of these factors include accessibility (including cost and availability), education level and income (Hassan \& Hekmat, 2012; Tovar et al., 2012; Chen, 2010).

Once someone immigrates, their diet begins to shift (Hassan \& Hekmat, 2012). Often the immigrant tries to choose traditional foods, but if those foods are not available, they substitute with the host's foods (Hassan \& Hekmat, 2012; Tovar et al., 2012). One study conducted on Chinese immigrants in the U.S. showed that the strongest predictor of dietary acculturation was the cost of food as well as the availability of healthy foods 
(Chen, 2010). If the foods they preferred were not widely available, or were too costly, they would likely shift to what they could afford. Education and income level play a role in dietary acculturation. Generally a higher income and education level is matched with more healthy dietary habits but this is not always the case. In a study of 65 ChineseAmerican children a higher household income was correlated with a higher maternal intake of fat and sweets. They also found that with an increase in maternal education there was an increase in physical activity (J. Chen, 2010).

Often, dietary acculturation leads to a mix of the two culture's diets and can lead to both positive and negative changes. A mixture of diets has been documented in female Arab immigrants after living in Toronto, Canada for at least 8 years. Their diets had a combination of Western and Arabic food. Some items that had been introduced into their diet from Canada included: "brown rice, whole wheat bread, whole grain flour, fish... broccoli, bagels, spaghetti, berries and soy.” They also switched from frying many foods to boiling, broiling and grilling them (Hassan \& Hekmat, 2012). This is an example of healthful changes that can occur during dietary acculturation. Alternately in a qualitative study of Brazilian, Haitian and Latin American women who had immigrated into the U.S. the authors found that the food the immigrants consumed in the U.S. wasn't as healthy as the food found in their country of origin. It was less natural, more processed and less fresh (Tovar et al., 2012). Another example of dietary change was measured between Korean-American women who were born in the U.S. and immigrant-Korean women. They found that the U.S. born Korean-American women consumed more discretionary fat, less fiber, less fruits and vegetables and more meat than their Korean born counter- 
parts. On the positive side, the U.S. born Korean-American women also consumed more nuts, less sodium and more yogurt (Park, Murphy, Sharma, \& Kolonel, 2005).

Dietary acculturation, especially in the U.S., can be concerning because many immigrants' health deteriorates as they acculturate (J. Chen, 2010). In the example of Korean women in the U.S., U.S. born Korean American women had higher BMIs than the Korean born women (Park et al., 2005). Another study showed that an increase in acculturation in Chinese, Korean and Vietnamese adults was associated with an increase in BMI (L. Chen et al., 2012). The association was strongest among the men (L. Chen et al., 2012). Another study of Japanese Americans showed that a diet high in Western foods was associated with an increase in BMI, type 2 diabetes and C-reactive protein, a sign of inflammation (Pierce et al., 2007).

\subsubsection{Dietary Acculturation in Mexican Americans}

Some of the most comprehensive research on dietary acculturation has been conducted on Mexican Americans, however the results are mixed. Discrepant findings are partly due to the variety of diets found within Mexico. When coming to the U.S., someone originating from a more urban area will change their diet less drastically than someone coming from a more rural area in Mexico, mainly because populations in the more urban parts of Mexico have already begun to consume more of a western diet whereas people in the more rural areas are more likely to consume the traditional Mexican diet (Pérez-Escamilla, 2009). Many cities in Mexico are showing trends in an increased consumption of meat and fast foods (Curiel, Levy, \& Vlliasana, 1995)

Traditional Mexican diets differ greatly from the American diet. The traditional diets contain a lot of fresh fruits, (Evans et al., 2011; Romero-Gwynn \& Gwynn, 1993) 
and beans which, when combined with corn, are a great source of protein and (Evans et al., 2011). Maize has been a staple in the Mexican diet, since the establishment of agriculture in Mexico. Maize has been predominately eaten as tortillas, in soups and tamales (Allen, 1992). Common vegetables traditionally consumed include "beans, squash, potatoes, nopales, tomatoes, peppers and herbs". Depending on the region, foods such as "pineapples, avocado, plums, peanuts and jicama" are also common. Many changes to the Mexican diet arrived with the Spanish in the $16^{\text {th }}$ and $17^{\text {th }}$ centuries. They brought the addition of many different meats (including cows, pigs and chicken) and grains (rice, oats, barely). They also brought some new seasonings/condiments to the Mexican diet including garlic, parsley and radishes (Allen, 1992).

There have also been some less healthy food practices in Mexico more recently. Soft drinks, fats (like lard), sweet breads, sugar and dairy products have become more popular as the years have progressed (Allen, 1992).

In general, as Mexicans acculturate in the U.S. they tend to eat less healthier foods and more "junk food". They consume: less fruits, vegetables and legumes (G. X. Ayala et al., 2008; Duffey et al., 2008; Montez \& Eschbach, 2008; Pérez-Escamilla, 2009), more fast food (Duffey et al., 2008), more soda (G. X. Ayala et al., 2008; Duffey et al., 2008; Pérez-Escamilla, 2009), more sugar (G. X. Ayala et al., 2008) and less fiber (Montez \& Eschbach, 2008). Intake of certain meats including bacon and sausage may increase with an increase in acculturation (Montez \& Eschbach, 2008). On a positive note, acculturated Mexican Americans tend to eat more high fiber bread, less fruit juice (Pérez-Escamilla, 2009) and eat less saturated fats (N Landale et al., 2000; RomeroGwynn \& Gwynn, 1993). 


\subsubsection{Dietary Acculturation in Mexican American Children}

Some studies have shown a relationship between increased acculturation and risk of childhood obesity (Hernández-Valero et al., 2007). One study conducted in Houston, Texas, on 438 Mexican American mothers with children between the ages of 5 and 18 years found that children of mothers born in the U.S. were twice as likely to be at risk for overweight or actually overweight (Hernández-Valero et al., 2007). However another study conducted in Chicago on 80 Mexican American children 5 to 6 years of age found that country of origin did not affect prevalence of obesity in the children (Ariza, Chen, Binns, \& Christoffel, 2004). Some of the conflicting findings may come from the differences in measurements of acculturation (Wojcicki, Schwartz, Jiménez-Cruz, Bacardi-Gascon, \& Heyman, 2011) as well as the differences in dietary acculturation based on what part of Mexico someone originated (urban versus rural).

Because of the high rates of childhood obesity within the Mexican American population, and the possible role of acculturation on childhood obesity, it is important to note how dietary acculturation affects children. There are few studies on dietary acculturation in children; the research is limited to older children and has conflicting findings. It seems that more acculturated children tend to consume more sodium (Liu et al., 2012; Pérez-Escamilla, 2009), less fruit (Liu et al., 2012; Morello, Madanat, Crespo, Lemus, \& Elder, 2012), less vegetables and beans and more saturated fat. Some beneficial changes include consumption of more whole grains and less meats than the less acculturated (Liu et al., 2012). Most of this research has been done on children 5 years and older and there have not been reports about dietary acculturation in younger children. 


\subsection{Acculturation and Body Image Perceptions}

Other factors that may be affected by acculturation are perceptions of body size and weight. Different cultures have different ideals of beauty and weight and different cultures hold different levels of importance for weight and body size (Brewis, 2003; Guendelman et al., 2010; Rosas et al., 2010).

\subsubsection{Maternal Perceptions of her Child's Body Weight}

Many factors contribute to the pediatric obesity epidemic however parents and caregivers have the strongest influence on their children's health (Boyington \& Johnson, 2004). Parental awareness and perception of their children's weight status can affect how they feed their child. For instance, one study found that African American mothers were more likely to introduce non-milk foods before two months of age if they believed their infant to be small at birth as opposed to large at birth (Boyington \& Johnson, 2004). In a study in Greece, mothers who underestimated their child's weight had children with higher energy intakes compared with mothers who were accurate in their perceptions of their child's weight (Manios, Moschonis, Grammatikaki, Anastasiadou, \& Liarigkovinos, 2010). If a mother has an incorrect perception of her child's weight, she is likely to feed $\mathrm{him} /$ her inappropriately. It is the parent's responsibility to intervene with diet and exercise if their child is overweight but parents with overweight children won't intervene if they are unable to see a problem.

Maternal perceptions of body weight are an interesting outcome to measure because these perceptions are somewhat abstract. Many studies have tried to measure maternal perceptions of children's body weight. In order to see if a mother perceives her child's body weight accurately one must first determine the child's actual body size, by 
measuring the child's weight and height and then determining their BMI, Z-score or weight for height percentile (De La O et al., 2001; Olvera et al., 2005; Petti \& Cowell, 2011). Two main methods are generally used to assess the parent's perception of their child's weight: verbal categorization and picture recognition (De La O et al., 2001; Olvera et al., 2005; Petti \& Cowell, 2011). In the first method mothers are asked to verbally categorize their children into categories which include underweight, normal weight/"about right", at risk of overweight (AROW), overweight and obese. Their perceived verbal category is then compared with the child's actual weight category (Chaparro et al., 2011; De La O et al., 2001). The second method uses sketches of children at different ages, genders and sometime races. The mother then points to the picture that best depicts her child or her ideal size for her child. Each image will have a BMI or height for weight category associated with it. The child's actual height for weight is then compared with the picture the parent referenced to determine if there is any discrepancy between the two (Eckstein et al, 2006, Olvera et al., 2005, Petti et al, 2011). Mothers tend to be more accurate when pointing to sketches than they are at verbally categorizing their children (Eckstein et al, 2006). A commonly used image scale was developed by Stunkard et al. and then adapted by Collins (M. E. Collins, 1991) but other scales have been made as well (Boyington \& Johnson, 2004). An example of these sketches is found below in Figure 2-2 (Boyington \& Johnson, 2004). 


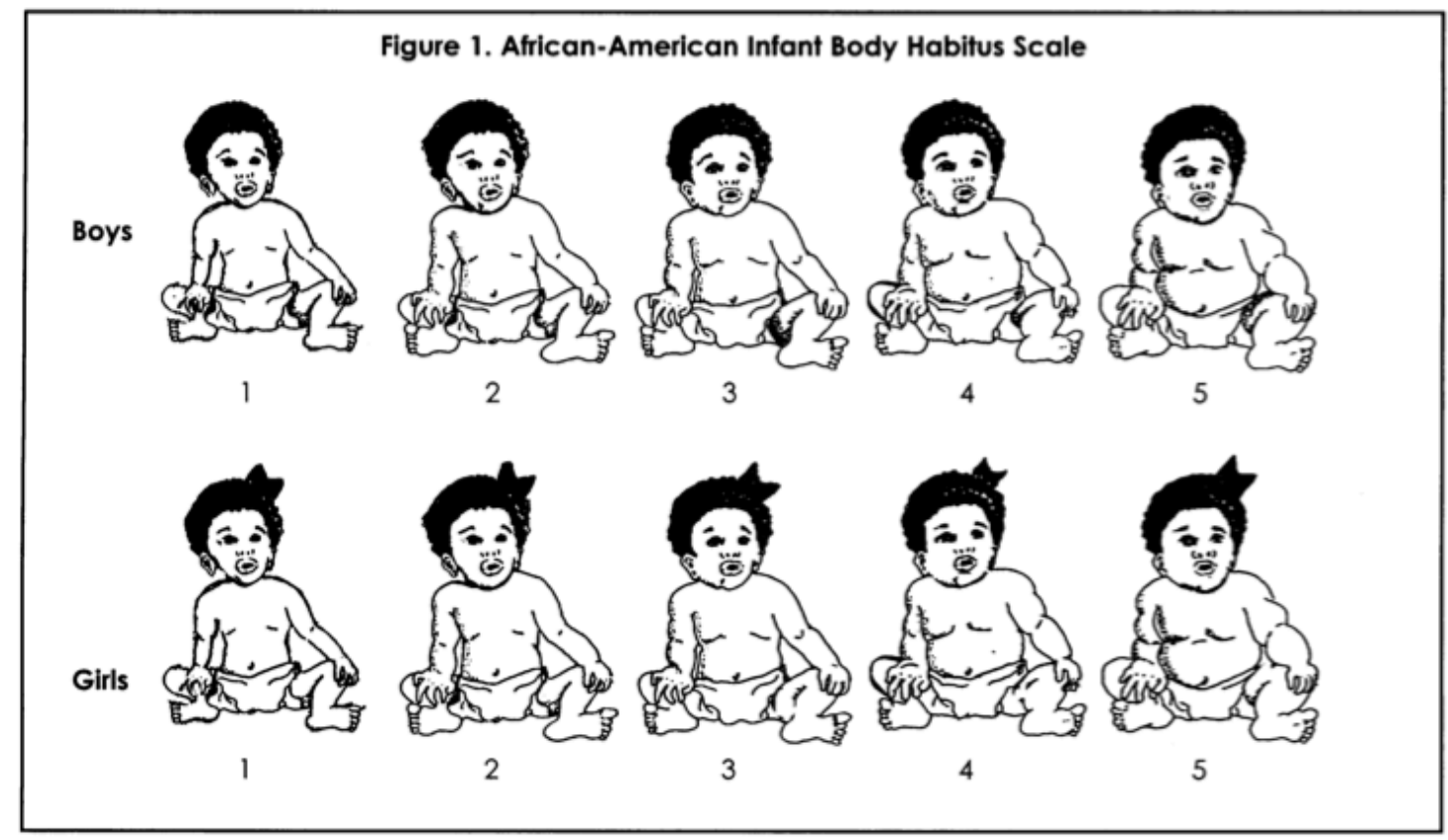

Figure 2-2. The African-American Infant Body Habitus Scale (AAIBHS). Excerpted from: (Boyington \& Johnson, 2004)

It has been demonstrated that parents often underestimate their child's actual weight especially when they have an overweight child (De La O et al., 2001; Eckstein et al., 2006; Olvera et al., 2005). Many factors influence maternal perceptions including culture. A study of an urban African-American population found that one-fifth of the mother's perceive their children to be larger than their ideal body size and $43 \%$ of mothers perceived their child to be larger than a typical child in their neighborhood but in general they estimated their child's weight accurately (Boyington \& Johnson, 2004). A study conducted on Latina mothers suggests they have similar perceptions as do the majority of American mothers in that they tend to underestimate their children's weight, however they may be more likely to underestimate their child's weight than most U.S. mothers. In a study of Hispanic mothers seen at a WIC clinic in Texas, $77.5 \%$ of mothers of obese children believed their child was at the right weight when in actuality, their child 
was obese compared with $28 \%$ of mothers of obese children in a mixed racial group (Chaparro et al., 2011; De La O et al., 2001). Ninety-two-point-six percent of Latina mothers of normal weight children, however, categorized them accurately as normal weight (Chaparro et al., 2011). These findings indicate that Latina mothers of overweight and obese children have difficulty in identifying their children as overweight or obese and may be more likely to underestimate their child's weight than the general population although this is not the case when their child is of a normal weight.

Many factors affect maternal perceptions of weight in their children, some of which are related to child factors and some that are maternal factors. Child characteristics shown to influence mothers' perceptions of their children's weight include the child's age, birth weight and rapid infant weight gain (Boyington \& Johnson, 2004; Chaparro et al., 2011; Huang et al., 2007; Manios et al., 2010). One study which interviewed 1098 mothers found that mothers are more accurate in categorizing children, whether they are their own or are unrelated to them, when they are older than 4 years of age and less accurate in younger children (Huang et al., 2007). Mothers tend to be less accurate in categorizing their children's weight if the child had a higher birth weight and were more likely to underestimate their child's actual weight (Chaparro et al., 2011). A study conducted in over 2500 children aged 1 to 5 in Greece found that mother's were more likely to underestimate their children's weight if their child had undergone rapid infancy weight gain (Manios et al., 2010).

Maternal factors that affect a mother's perception of her child's weight include: environment (urban versus rural), education level, maternal BMI and possibly, acculturation (Guendelman et al., 2010, Manios et al., 2010, Boyington \& Johnson, 2004, 
Chaparro et al., 2011). One study found that Hispanic women living in urban areas of California have different perceptions of body sizes than Hispanic women in rural areas. For instance, Hispanic mothers in rural areas believed that an overweight infant would outgrow their weight whereas Hispanic mothers in more urban areas made no mention of that belief (Guendelman et al., 2010). A study in Greece (Manios et al., 2010) and another in the U.S. (Huang et al., 2007) showed that less than a high school degree was associated with an increased likelihood of underestimating their child's body size. However, two other studies show no correlation between education and accuracy (Chaparro et al., 2011; Olvera et al., 2005).

Multiple studies have suggested that a mother's BMI is correlated with her accuracy in estimating her child's body size (Boyington \& Johnson, 2004; Huang et al., 2007; Manios et al., 2010). Generally, an increase in maternal BMI increases the likelihood of underestimating their child's weight (Boyington \& Johnson, 2004; Manios et al., 2010). The reasons why a mother's BMI affects her perceptions has not been discussed very much in the literature, however, it has been suggested that mothers of higher BMI may believe a heavier weight to be healthy or may believe their child will outgrow their weight (Manios et al., 2010). Cultural norms and acculturation are more factors that may or may not affect a mother's perception of her child's body size.

In the Mexican culture it is believed that a chubby baby is a healthy baby (Guendelman et al., 2010; Rosas et al., 2010). This view is due in part to the history of food insecurity in Mexico. In places where food is scarce, a chubby child shows that your child has enough to eat. These children would look healthier compared to other thin, malnourished children (Brewis, 2003). While a chubby baby ideal in Mexico may have 
traditionally been associated with survival, in the U.S. this cultural ideal may lead to health risks later in life. Focus groups with mothers currently living in Mexico and with mothers of Mexican origin living in California found that both mothers had similar abilities to perceive their child's actual weight (Guendelman et al., 2010). However, mothers currently residing in Mexico tended to prefer a larger child than the mothers living in California (Guendelman et al., 2010). This was attributed to the Mexican cultural belief that a chubby baby is a healthy baby which is related to food security in Mexico (Guendelman et al., 2010, Rosas et al., 2010). In contrast, another study showed that Mexican-American mothers in California were more likely to underestimate their child's actual body size than mothers living in Mexico (Rosas et al., 2010). The Californian residents' increase in underestimation may have been due to the higher prevalence of overweight among the children in the U.S. compared with Mexico. Nearly $42 \%$ of the children living in the U.S. were overweight as opposed to only $6.7 \%$ of the children living in Mexico (p-value $=0.01)($ Rosas et al., 2010). These studies suggest that perceptions change when mothers immigrate from Mexico to the U.S.. One study found that the higher the mom's level of acculturation, the thinner the daughter offspring desired to be, while this correlation did not hold true with the boys (Olvera et al., 2005). Another study looked at several variables simultaneously including child's gender and birth weight and mother's BMI, education, acculturation level and language preference and their effect on maternal perceptions. This study found tat after controlling for other variables acculturation was not a significant factor but that maternal BMI and child's birth weight were significant factors affecting maternal perceptions of their child's body 
size. As mother's BMI and child's birth weight increased she was more likely to underestimate her child's weight status (Chaparro et al., 2011).

A mother's desired body weight for her child could also affect how she approaches the issue of obesity since if a mother preferred her child to be bigger, she may not be concerned with an overweight child or may be feeding them more to get her child to obtain her ideal body weight for the child (Boyington \& Johnson, 2004). Information on Hispanic-American mother's desired body size for their children is confusing at best. One study conducted with 80 Hispanic women with older children showed Hispanic mother's desired body size for their children correlated well with their child's actual body size. In other words, the mothers were content with whatever weight their child was at (Olvera et al., 2005). A focus group with mother's living in Mexico found that the mothers preferred a large or chubby baby and claimed chubby babies were healthy and cute however they did not prefer an overweight child (Guendelman et al., 2010). These findings are based on children at different ages, which may be why the data seems inconsistent.

A child's age may influence a mother's desired body size for her child. Focus groups with Hispanic mothers have revealed that parents are more concerned by an older overweight child (toddler and up) than an infant (McDermott, Hey, Teaford, \& Minarik, 2009). It was disclosed that most parents believed a doctor should intervene if an infant was overweight but thought the family shouldn't worry about overweight children until the children were toddlers. They believed it was too early to identify an infant as overweight since they aren't mobile (McDermott et al., 2009) . Age-related inconsistency of mother's perceptions was also shown in a study of mothers in Mexico. Mothers 
believed an overweight infant was desirable but a chubby child was less acceptable (Guendelman et al., 2010). And yet another study of parents of all ethnicities showed that parents were more likely to recognize an older child ( $\geq 6$ years old) to be overweight and were more likely to be concerned about this (Eckstein et al., 2006).

Although there are many factors interrelated with varying maternal perceptions, these do not explain why Hispanic mothers perceive their children incorrectly. There are many theories on what might be causing these misconceptions (Chaparro et al., 2011; Guendelman, Fernandez, Thornton, \& Brindis, 2011; Huang et al., 2007). It may be that it is difficult to identify obesity because of the growing number of obese children in the child's environment, so an overweight child looks normal when compared to peers (Chaparro et al., 2011). There are a couple theories as to why Hispanic American mothers have more difficulty with their perceptions of younger children. Firstly, the cultural belief that a chubby baby is a healthy baby and a thin baby is associated with food insecurity and decreased survival may affect mother's perceptions and desire for an overweight infant or toddler (Chaparro et al., 2011). Second, many Hispanic mothers believe genetics is the most important factor determining weight and that their child will grow into their weight as they get older (Guendelman et al., 2010).

In summary, research has shown that mothers tend to underestimate the weight of their children (De La O et al., 2001; Eckstein et al., 2006; Olvera et al., 2005). Underestimation is especially likely if the child had a high birth-weight (Chaparro et al., 2011) and is younger than 4 years of age (Huang et al., 2007). Other factors influencing perceptions include mother's environment (living in an urban versus rural area) (Guendelman et al., 2010), lower education levels (Huang et al., 2007; Manios et al., 
2010), higher BMI (Boyington \& Johnson, 2004; Manios et al., 2010) and possibly acculturation level (Guendelman et al., 2010, Rosas et al., 2010).

A mother's ideal body weight for her child could also affect how she approaches the issue of obesity. However, Hispanic mother's ideal body weight for their children is unclear (Boyington and Johnson, 2004; Chaparro et al., 2011; Guendelman et al., 2010; Olvera et al., 2005).

\subsubsection{Maternal Perceptions of Self}

Many of the same issues of maternal misperceptions of children's body weight carry over to a mother's inaccurate view of herself. If the mother does not realize she is overweight, how will she know she should change her diet or level of physical activity? When looking at a mother's perceptions of her own body weight, several studies have compared what a mother believes her body looks like versus her actual weight category using BMI (Lopez, Blix, \& Blix, 1995; Petti \& Cowell, 2011). There is some evidence that Latina mothers tend to underestimate their own body weight, which is similar to their under estimation of their children's body weight. Lopez showed that non-Hispanic white mothers and Latina mothers who had immigrated to the U.S. before the age of 16 underestimated their body weight but non-Hispanic white and Latina mothers who moved to the U.S. later on perceived themselves accurately. This suggests a relationship between acculturation and maternal perceptions of body weight (Lopez et al., 1995). Alternatively, another study of 35 Mexican American mothers found that the mothers chose a figural body size that was very close to their actual body size (Petti \& Cowell, 2011).

Across all cultures and women of all ages, overweight women tend to have a high rate of body dissatisfaction (Fitzgibbon, Blackman, \& Avellone, 2000; Guinn, Semper, 
Jorgensen, \& Skaggs, 1997; Lopez et al., 1995). Body dissatisfaction is the difference between mother's desired body weight measured using picture scales or verbal categorizations and their actual body weight (Lopez et al., 1995). One study that looked at differences in body dissatisfaction between white, Hispanic and African American women found that the point at which a woman would become dissatisfied with her weight differed by ethnicity. White women became dissatisfied with their body at a BMI of 24.6 $\mathrm{kg} / \mathrm{m}^{2}$ ( just below the cut off of $25 \mathrm{~kg} / \mathrm{m}^{2}$ for overweight), whereas black women became dissatisfied at a BMI of $29.29 \mathrm{~kg} / \mathrm{m}^{2}$ and Hispanic women became dissatisfied at 28.57 $\mathrm{kg} / \mathrm{m}^{2}$. These later two BMIs are at the higher and middle end, respectively, of the overweight category (Fitzgibbon et al., 2000). This suggests that black and Hispanic women are more comfortable with a larger body size than white women and this may be one factor that accounts for their higher rates of obesity (Fitzgibbon et al., 2000). Differences in body dissatisfaction between ethnicities may be due to different cultural ideas of beauty. Hispanics and African Americans may view a larger body size as more beautiful (Grabe \& Hyde, 2006; Petti \& Cowell, 2011).

\subsection{Acculturation and Stress}

Latinos who have recently immigrated to the U.S. have additional stressors compared to the rest of the general population (Garcia \& Lindgren, 2009; Marsiglia, Kulis, Perez, \& Bermudez-Parsai, 2011). Many have separated from their families to immigrate, have difficulty finding suitable jobs and health insurance, and face the challenges of learning a new language and culture (Marsiglia, Kulis, Perez, \& BermudezParsai, 2011). It is also common for this population to experience racism and discrimination, violence in their neighborhoods and fears of deportation if they are in the 
U.S. illegally (Garcia \& Lindgren, 2009). Although the immigrant population from Mexico faces many challenges, they seem to perform better on mental health tests than European American women (Acevedo, 2000). For instance, one study conducted on 331 Mexican American women and 263 European American women found that the Mexican American women had better scores on the Revised Mental Health Inventory than the European American women. Low acculturation in the Mexican American population may play a protective role in regards to mental health. The Mexican culture in general values community and interpersonal relationships which may help mitigate stress (Campos et al., 2007). Also it is possible that low acculturated Mexican Americans find their current situation to be more favorable compared to their life in Mexico (Eschbach, Ostir, Patel, Markides, \& Goodwin, 2004). Campos et al. (2007) looked at 1,064 Latina women to observe their perceived stress, pregnancy anxiety and infant birth weight. The authors found that the higher the acculturation or more "Anglo orientation", the more perceived stress was reported ( $\mathrm{p}$-value $<0.05$ ). The authors also found that Mexican orientation was correlated with a lower degree of stress ( $p$-value $<0.001$ ) suggesting that being of "Mexican orientation" (or less acculturated) may have protective effects against stress but that the process of adapting to a new culture, much like what the Anglo oriented women experienced, invokes more stress (Campos et al., 2007).

Parenting stress can be defined as stress "resulting from perceived discrepancy between demands pertaining to parenthood and personal resources" (Ostberg, Hagekull, \& Hagelin, 2007). Parenting stress has been measured using a variety of different indexes. Some tests which are frequently used include the Parenting Stress Index (PSI), and the PSI Short Form (PSI-SF) (Abidin, 1983; Ostberg et al., 2007; Skreden et al., 
2012; Solis \& Abidin, 1991). The PSI has a total of 120 questions, which evaluates 3 domains: the child domain score (54 questions), parent domain score (47 questions) and life stress domain score (19 questions) which is an optional set of questions. Questions have answers ranging from "Strongly Agree" to "Strongly Disagree". The PSI covers many areas of possible stress for the parents. Under the child domain score there are 6 domains of stress: adaptability, acceptability, demandingness, mood, distraction/hyperactivity and reinforces parent. Under the parent domain score there are 7 domains of stress: depression, attachment, restriction of role, sense of competence, social isolation, relationship with spouse and parent health (Abidin, 1983; Solis \& Abidin, 1991). The PSI-SF has been adapted from the original PSI and contains only 36 questions, which include questions on parental distress (12 questions), parent-child dysfunctional interaction (12 questions) and difficult children (11 questions). This test was made to take only 10 minutes to complete and is much simpler to score than the original PSI but does not contain as many stress related factors as the PSI (Abidin, 1983). The PSI-SF has been tested for validity a couple of times. Abidin (1995) tested the PSISF for validity among two samples of primarily white, married mothers and found that the scores on the long and short forms were highly correlated $(r=0.87)$. An additional study of over 200 mothers found that the PSI-SF was valid for two scales: a mother characteristics domain $(r=.54 \mathrm{p}<.001)$ and child characteristics domain $(r=.23 \mathrm{p}<.01)$ (Haskett, Ahern, Ward, \& Allaire, 2006).

Although research has looked at the effect of culture and acculturation on stress, there has been little investigation into cultural factors and parenting stress. Ostberg and colleagues (2007) used the PSI with parents of children with hyperactivity and found that 
ethnicity had no effect on parenting stress level but that parent's who spoke primarily Spanish did have higher levels of parenting stress in two domains: role restriction and sense of less competence. Role restriction involves feelings of closeness to the child such as "Sometimes I feel my child doesn't like me and doesn't want to be close to me" and "My child smiles at me much less than I expected". Examples of competence questions include: "My child doesn't seem to learn as quickly as most children" and "When my child came home from the hospital, I had doubtful feelings about my ability to handle being a parent" (Gupta, 2007). The authors concluded that difficulty with the English language may increase some areas of parenting stress and that translating services should be given to these families (Gupta, 2007).

Other factors that seem to increase parenting stress include: low education levels, higher number of children in the household, and low family income (Dabrowska \& Pisula, 2010; Smith, Ph, Oliver, \& Innocenti, 2001). Parenting stress has been seen to have a negative effect on a variety of conditions ranging from marital quality to child abuse (Ostberg et al., 2007). Since parenting stress has so many negative consequences and may be influenced by Mexican culture, it is an important issue to study.

\subsection{Objectives}

Since acculturation may have a role in many different health outcomes, this study aims to determine the association between the level of acculturation of low-income Latina mothers and foods consumed by their children ( 0 to 36 months old). The association between acculturation and maternal perceptions of infant and toddler body size, maternal perceptions of their own body size and maternal stress will also be investigated. 


\section{CHAPTER 3}

\section{MATERIALS AND METHODS}

\subsection{Overall Goals}

This research sought to determine the impact of acculturation in low income Mexican American women on foods offered to children that may contribute to obesity, on parental perception of body size and on parenting stress. Our end objective was to identify several key messages that would be appropriate for use in the WIC or Head Start populations to improve nutritional status and quality of dietary intake and reduce the risk of overweight and obesity in children.

\subsubsection{Aim 1}

To determine the association between acculturation in low-income Latina WIC mothers and specific factors that contribute to obesity in a cross-sectional study.

Participants: Sixty-four Mexican American mothers of children aged 0-36 months enrolled in the Supplemental Nutrition Program for Women, Infants and Children (WIC) in San Luis Obispo County.

Objective 1A: Determine mother's level of acculturation.

Objective 1B: Describe the frequency of consumption of "less healthy foods" and "healthy foods" in infants 0 to 19 months and children 19 to 36 months old. Within these groups determine whether there is an association between frequency of consumption of "less healthy" and "healthy" foods by level of acculturation in their mothers. Hypothesis of objective 1B: mothers who are more acculturated will have children who consume more of the "less healthy" food items and less of the "healthy" food items. 
Objective 1C: Determine whether there is an association between acculturation and maternal perceptions of her own body size as well as that of her child and determine the average ideal weight in Hispanic children.

Hypothesis of objective 1C: Less acculturated mothers will be more likely than more acculturated mothers to underestimate their own body weight as well as that of their child.

Objective 1D: Determine whether there is an association between acculturation and parenting stress.

Hypothesis of objective 1D: More acculturated mothers will have higher levels of parental stress than less acculturated mothers.

\subsubsection{Aim 2}

Develop focus group questions on acculturation, foods offered to children and maternal perceptions of body size using the results from Aim 1 and interviews of Key Informants. Participants: Nine WIC, Head Start, and public health professionals who work with the Mexican American population.

\subsubsection{Aim 3}

Conduct focus groups to gain a better understanding of findings in Aim 1 and to develop nutrition education messages for WIC.

Participants: Thirty-two Mexican American mothers of children 0-42 months enrolled in WIC or Head Start. 


\subsection{Materials and Methods: Aim 1}

Mothers were recruited, between March $10^{\text {th }}, 2011$ to May $10^{\text {th }}, 2011$, from the WIC center in Grover Beach during a regularly scheduled clinic visit and were requested to return 1 month later at a regularly scheduled WIC appointment. WIC staff identified potentially eligible mothers and referred them to the research staff to provide additional study information. Mothers were informed of the study details including any possible risks and benefits and if they agreed to participate, they signed an informed consent in their preferred language (Spanish or English) (found in Appendix A). Eligibility criteria included: women who self identified being of Mexican descent with a child who was between 2 months and 3 years of age, the child was without any congenital anomalies and able to consume all foods and/or liquids by mouth. In addition, the mother had to live within 10 miles of the WIC office in Grover Beach. In order to reach a target of 80 participants, with an anticipated 30\% drop out rate, 109 mother-child dyads were recruited. An attempt was made to enroll mothers with children equally distributed among 2-3, 4-6, 7-9, 10-12, 13-15, 16-18, 19-21, 22-24, 25-27, 28-30, 31-33 34-36 month age groups. Mothers were called 3 days prior to their scheduled visit to remind them of the study visit.

At the study visit, mothers provided information in their preferred language (Spanish or English). Anthropometrics (weight in pounds and height in inches) were obtained from the mothers, infants and children. Questionnaires included the following: Maternal perception of body size, Early childhood behavior, Parenting feeling index, and Food frequency. Each questionnaire was used to fulfill a different research objective and will be described later in detail. Questionnaires were designed so that all study forms 
could be completed in a 60-minute visit. The cross-sectional interviews occurred between May $2^{\text {nd }}$ and June $15^{\text {th }}$ of 2011.

This study was approved by the Cal Poly Human Subjects Committee. All participants provided written and verbal informed consent in Spanish or English according to their preference. Free child care was provided during the study visit. The women received a 10 dollar Walmart gift card at the end of the clinic visit to compensate for their participation time.

\subsubsection{Demographics and Anthropometrics}

Nutrition and demographic information on participants was obtained from San Luis Obispo (SLO) county WIC central office including: family income, country of origin, marital status, family size, employment status and mother's level of education. The mother and child's date of birth, age, current height or length (inches), and weight (pounds) were measured on the day of their scheduled visit. Mother's BMI and children's height-for-weight percentiles were calculated. Mothers were considered overweight if they had a BMI between 25.0 and 29.9 and obese if they had a BMI of 30.0 or greater. Infants and children were considered overweight at a height for weight percentile between $85 \%$ and $95 \%$ and obese at a $95 \%$ or higher using the CDC growth charts.

\subsubsection{Objective 1A: Acculturation}

Acculturation was measured using two items, language preference and country of origin. The language the mother preferred to complete the questionnaires in was considered her primary language. Since many of the mother's country of origin was not available in the WIC files, mothers were called about 6 months after the study had been conducted to obtain this information. We were able to obtain information on country of 
origin from 68 out of the 84 original mothers. These 68 women were used in the analysis of the present study. The mothers were then placed in their acculturation category and were considered "highly" acculturated if they chose to complete the questionnaire in English or if they were born in the U.S. and considered "lowly" acculturated if they chose to complete the questionnaire in Spanish and were born in Mexico. If they preferred Spanish but were born in the U.S. they were placed in the "high" acculturated category, similar to methods used by Liu et al. and Montez \& Eschbach $(2012,2008)$.

\subsubsection{Objective 1B: Food Frequency}

In order to determine the frequency of foods consumed by the children, mothers completed a semi-quantitative food frequency questionnaire (FFQ) based on their child's age (0-18 months or 19-36 months) (Appendix B). The FFQ was composed using a combination of two other FFQ's directed toward children and toddlers (Fein et al., 2008; Hurley, Black, Papas, Caulfield, \& Caufield, 2008). Once constructed, the FFQ was tested on 10 mothers with young children to ensure it's readability, difficulty and time to complete. Some changes were then made in order to increase understanding. WIC staff also gave input and additional food items were included accordingly.

The FFQ asked questions on who primarily feeds the child, whether the child had been breast fed and duration of breast feeding as well as questions on the frequency of consumption of fruit juice, soda, breakfast cereals (both sweetened and unsweetened), vegetables, fruit, protein items and foods prepared by a restaurant. The main difference between the FFQ for infants 0-18 months and the one for 19-36 months was that the one for infants included baby food options and infant formula whereas the 19-36 month questionnaire excluded these additional items. Sixteen undergraduate students studying 
nutrition and psychology were trained on administering the FFQ's. They had to practice and pass a test for competency before being allowed to administer the forms. The trained research assistants administered the FFQ by reading aloud each of the questions from his or her own sheet and then giving examples of each of the food items, while the participant followed and then answered the question on her own questionnaire. To avoid embarrassment or bias, researchers were instructed not to look at or help with the participant unless the participant asked for help.

Three food items were categorized as healthy foods including: fruits, vegetables and legumes. $100 \%$ fruit juice was not included due to its controversial nature; small amounts are considered nutritious, but excessive amounts are not. WIC advises mothers to keep juice consumption down to 4 ounces or less a day (The California WIC Program, 2010). Meat and dairy options were not included in this analysis because protein intake in toddlers in the general population is adequate (Briefel et al., 2006). There were 6 items included in the "less healthy" food composite score including: sweet drinks, soda, sugar cereals, French fries, fast food and sweets. Foods in healthy and less healthy categories are shown in Table 3-1. Possible answers for the FFQ included: never, rarely (once a week), about once a day, 2-3 times a day or every feeding. These were then converted into continuous variables as times per week: $0,1,7,18$ or 21, respectively. Each person's frequency per week scores for each food item within each category (healthy or less healthy) were added together for a composite score for each category. Total possible scores ranged from 0 to 63 for healthy foods and 0 to 126 for the less healthy foods. For example, if a child ate vegetables everyday ( 7 times per week) fruit rarely (1 time per 
week) and legumes 2-3 times a day (18 times per week) their "healthy" foods score would have been 26 .

Table 3-1 Food items used to calculate the "health" foods composite score and the "Less healthy" foods composite scores.

\begin{tabular}{l|l}
\hline "Healthy" Food Items & "Less Healthy" Food Items \\
\hline Any Vegetable & Sweet drinks \\
Any Fruit & Soda \\
Beans, Legumes & Sugar cereals \\
& French fries \\
& Fast food \\
& Sweets
\end{tabular}

To determine the frequency of consumption of each of the food items measured for each of the acculturation levels, t-tests were used (JMP, Version 10.0, SAS). To determine the effect of acculturation on food frequency for each food category (healthy versus less healthy) General Linear Regression was used (Minitab, Version 16, Microsoft). The variables measured had an unequal spread of variance so the square root of food scores for both less healthy and healthy food items was used. The following continuous variables: child's age (months), mother's age (years), mother's BMI ( $\left.\mathrm{kg} / \mathrm{m}^{2}\right)$ and education level (years) were added to the model as control variables. Significance was set at a p-value of $<0.05$.

\subsubsection{Objective 1C: Maternal Perceptions of Self}

Mothers were shown figures, developed by Stunkard et al., that represent women at different BMIs (Figure 3-1) (Stunkard, Sorenson, \& Schulsinger, 1983). They were then asked which of the figures they thought best represented their own body, which figure represented a typical Hispanic woman in their neighborhood, which figure represents a healthy body size and which figure they preferred to look like. The study staff recorded the mother's response. These questions were similar to another study 
conducted on African American women (Boyington \& Johnson, 2004). First, an unadjusted analysis was run to determine the mean figure score chosen by each of the mothers for each of the 4 questions. Answers were compared by acculturation level using t-tests (JMP, version 10, SAS).

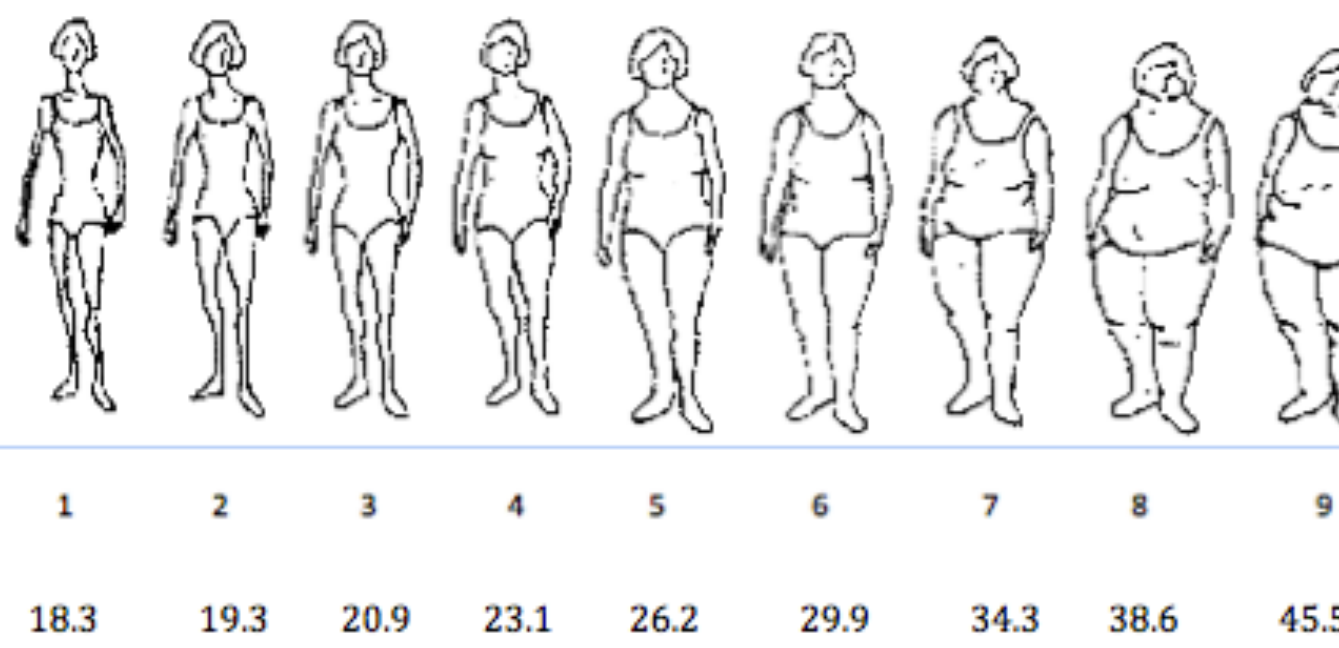

Figure 3-1 A silhouette scale developed by (Stunkard et al.) used to assess maternal perceptions of body size. Below each score are the correlating BMIs. Figural Rating Scale (Stunkard et al., 1983).

Each female figure has a score associated with it, ranging from 1 to 9 , and was correlated with a specific BMI or BMI range. A score of 1 was equivalent to a BMI of $\leq 18.3 \mathrm{~kg} / \mathrm{m}^{2}$ scores of 2 to 4 were equivalent to about normal weight with a BMI of 18.4$24.5 \mathrm{~kg} / \mathrm{m}^{2}$; a score of 5 to 6 was equivalent to about overweight with a BMI of 24.6-28.0 $\mathrm{kg} / \mathrm{m}^{2}$; and a score of 7 to 9 being very overweight and obese with a BMI of $\geq 28.1 \mathrm{~kg} / \mathrm{m}^{2}$. To determine how accurate the mother was in predicting her own body size the difference between the score the mother chose as her perceived body size was subtracted from the score that corresponded with her actual body size. For instance, if a mother stated she 
looked like the figure labeled number 5, but her actual BMI correlated to figure number 6 she would receive a score of -1 (perceived 5-actual 6). To determine the effect of acculturation on accuracy of perceptions, the General Linear Regression model (Minitab, version 16, Microsoft) was used controlling for the following continuous variables: acculturation level (high or low), child's age (months), mother's age (years), BMI $\left(\mathrm{kg} / \mathrm{m}^{2}\right)$ and education level (years).

\subsubsection{Part 2b: Maternal Perceptions of her Child}

Mothers were shown 2 sets of figures, one of which was adapted from an African American scale by Boyington et al. (2004) in order to make infants appear more racially generic. The toddler figures used were created by a Cal Poly student. The figures represented different height for weight percentiles for infants (age 0 to 11 months) (Figure 3-2) and for toddlers (aged 1 to 3 years) (Figure 3-3). Each figure has a score associated with it, ranging from 1 to 5 . Each score/figure was representative of a specific height for weight percentile range with a score of 1 representing a weight for height percentile of $<10 \%$, a score of 2 was equivalent with a weight for height percentile of 10$25 \%$, a score of 3 with a weight for height percentile of $26-74 \%$, a score of 4 with a weight for height percentile of $75-85 \%$, and a score of 5 with a weight for height percentile of $>85 \%$. The mothers were asked which of the figures they thought best represented their own child's body, which figure represented a typical Hispanic child in their neighborhood, which figure represents a healthy body size for a child of that age and which figure they preferred their child to look like. The study staff recorded the mothers' responses. 
The difference between the score the mother chose as her perceived body size for her child was subtracted from the score that corresponded with her child's actual body size to determine how accurate the mother was in predicting her child's body size. For instance if a mother stated her child looked like the figure labeled number 4, but the child's actual weight for height correlated to figure number 5 she would receive a score of -1 (4 perceived - 5 actual) which would mean that she underestimated her child's weight. To determine the effect of acculturation on accuracy of perceptions, the General Linear Regression model (Minitab, version 16, Microsoft) was used controlling for the following continuous variables: child's age (months), mother's age (years), BMI ( $\left.\mathrm{kg} / \mathrm{m}^{2}\right)$ and education level (years).
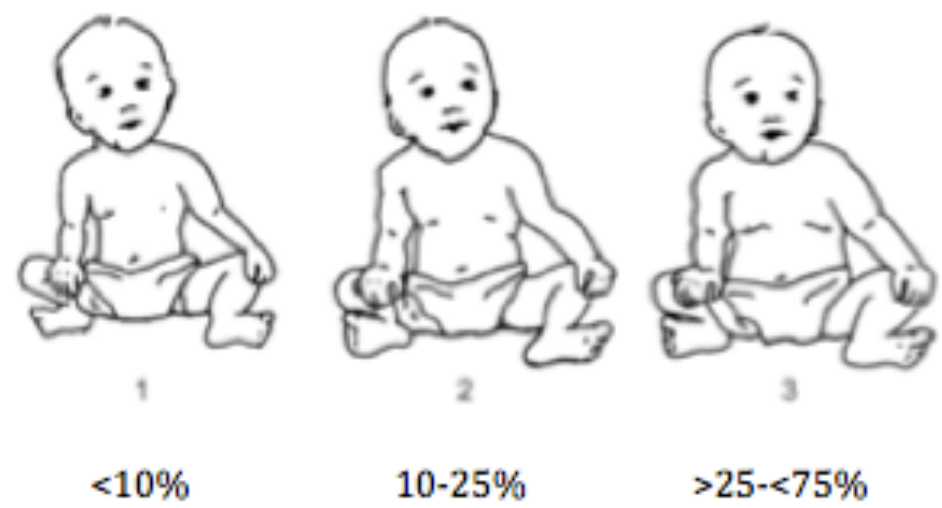

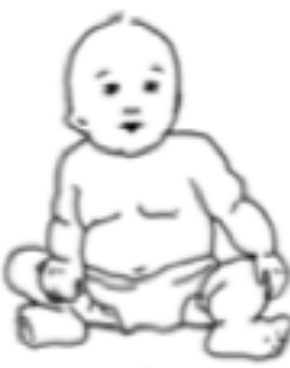

4

$75-85 \%$

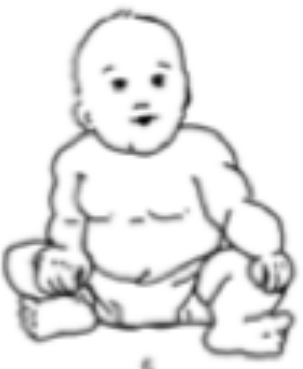

s

$>85 \%$

Figure 3-2 Infant figures adapted from Boyington et. al. Silhouette scale used to assess maternal perceptions of infant's body size (age 12 months and under). Corresponding height for weight percentiles are located under the figure's score (Boyington \& Johnson, 2004). 


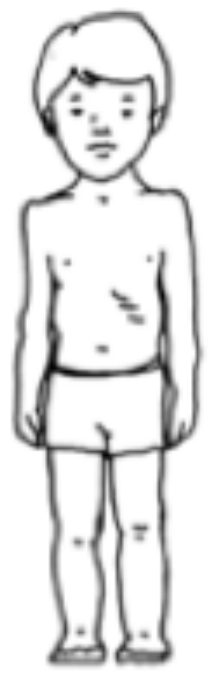

1

$<10 \%$

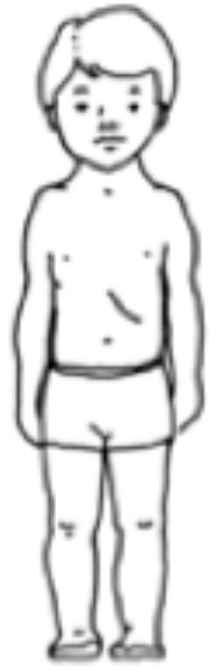

2

$10-25 \%$

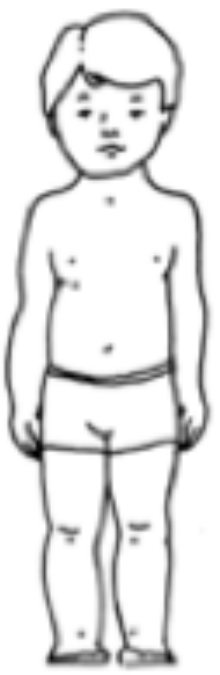

3

$>25-<75 \%$

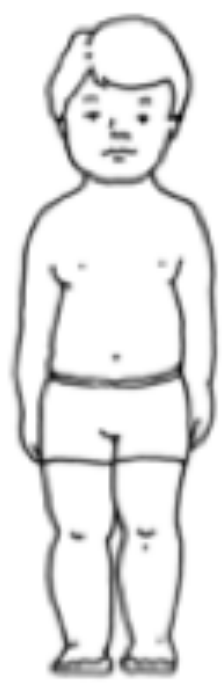

4

$75-85 \%$

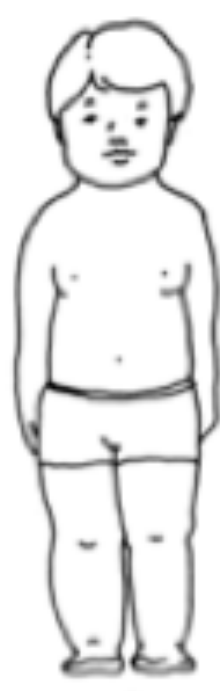

5

$>85 \%$

Figure 3-3 Toddler figure scale developed by Erica Melling. Used to assess maternal perceptions of her toddler's (age 1-3) body size. Corresponding height for weight percentiles are located under the figure's score.

\subsubsection{Objective 1D: Parenting Stress Levels}

Participants were assisted in the completion of a form called the Parenting

Feelings Index, which included 44 questions and can be found in the Appendix D, based on the Parental Stress Index-Short Form (PSI-SF) (McKelvey et al., 2009; Abidin et al., 1995; Solis and Abidin,1991). It was adapted by Patrice Engle, PhD (study co-principal investigator) and re-named the Parenting Feelings Index. The original PSI-SF was thought to be too negative in tone so some questions were changed to be more positively worded and 8 additional positive questions were added. For scoring the test, the questions that were changed were given a reverse score and the 8 added positive questions were eliminated since they were not included in the original PSI-SF and had not been validated. Answers from the original PSI-SF originally ranged from "Strongly Agree" to "Strongly Disagree" whereas the adapted form had answers ranging from "Almost 
Always" (a score of 4) to "Does not Apply" a score of 0). A score of 0 correlated with the lowest level of parenting stress and a score of 4 correlated with the highest level of parenting stress. Two sub scores were present: questions about the child and questions about the parents. This also differed from the PSI-SF, which had 4 domains. Many of the mother's circled 0 for a majority of their answers, which was correlated with "does not apply". After the study it was decided to group the 0 and 1 scores together and call them all "Rarely" giving the questions a score of 1 instead of 0 for those who selected 0 . Many of the mothers left some questions unanswered so the total sum of the answered questions were calculated and then averaged for each mother. For example, if a mother answered only 34 of the 36 questions, her answers were summed and then divided by 34 (the total number of question she answered) to create her average score. To determine the effect of acculturation on parenting stress these average scores were then compared between the two acculturation groups controlling for child's age (months), mother's age (years), BMI $\left(\mathrm{kg} / \mathrm{m}^{2}\right)$ and education level (years) and a linear regression model was fit (Minitab, Version 16, Microsoft).

This adapted Parenting Feelings Index has not been tested for validity. It is very similar to the PSI-SF in that it contains most of the same questions but different options for the answers (e.g. "almost always" instead of "strongly agree"). For both the PSI-SF and this new Parenting Feelings Index a higher score is correlated with high stress and a lower score is correlated with low stress making responses to the forms comparable.

\subsection{Aim 2: Key Informant Interviews}

In order to help develop questions for our focus groups, we conducted key informant interviews with 4 Head Start professionals, 3 WIC dietitians and 2 Public 
Health nurses. These individuals were identified by senior program management at WIC and Head Start due to their bicultural experience. The interviews were approved by the Cal Poly Human Subjects Committee. All participants provided written informed consent (Appendix F).

Interviews were conducted by this author one-on-one at the professional's work place. All of the interviews lasted from 30 minutes to 1 hour and were audio recorded. Specific questions were asked but participants were encouraged to talk about related areas they thought could further the research.

During the individual interview the participant was informed about the earlier data analysis and asked a series of questions (Appendix G). The questions involved knowledge about the effect of acculturation on foods given to children, levels of maternal stress and maternal perceptions of healthy and desirable body types for both themselves and their children. The interviews were then transcribed by the author and assessed for common themes.

\subsection{Aim 3: Focus Groups}

Mothers for the focus groups were recruited from a WIC clinic in Grover Beach, a Head Start Center in Five Cities and a Head Start Center in Paso Robles. Inclusion criteria for focus groups were self-identification of being of Mexican descent, having a child who was 3.5 years or younger and participation in WIC or Head Start. In order to recruit 6-8 mothers each for 8 focus groups (goal $=2$ high acculturation and 2 low acculturation groups for WIC participants and 2 high acculturation and 2 low acculturation for Head Start participants), a total of 107 mothers were recruited. 
Recruitment occurred over the course of about 2 months from October of 2012 to November of 2012. A member of the research team went to the WIC clinic during the day and to Head Start Centers during key times when parents pick up and drop off their children. Mothers were given an interest explanation form (Appendix I) in either English or Spanish, whichever they preferred, which explained what the research entailed and what could be gained from it. If the mothers were interested and met the inclusion criteria they were asked to sign an interest form giving consent to release of some personal information (Appendix J). The mothers then completed a bi-dimensional acculturation scale questionnaire with a short demographic questionnaire which included questions on the mother's age in years, the number of children living at home, their marital status, employment status and their country of origin (Appendix L).

The Bi-Dimensional Acculturation Scale (BAS) asks 24 questions about language use. These questions are divided into two subscales- Hispanic Domain and Non Hispanic Domain, each containing 12 questions. Scores were totaled and then divided by the number of domain questions (12) for each domain; a score of 2.5 or more for the Hispanic domain places the participant in the low acculturation group. A score of 2.5 or more in the Non Hispanic Domain categorizes the participant as high acculturation and a score of at least 2.5 in both categorizes the person as bi acculturation (Marin \& Gamba, 1996).

Bilingual recruiters were trained on conducting the BAS. Each recruiter practiced asking the questions on the BAS in both English and Spanish and on scoring the scale. The recruiters were not allowed to perform the BAS until they had successfully conducted a practice test and scored it properly in both Spanish and English. After 
administration of the scale in the mother's preferred language, the recruiter then scored the test to determine which acculturation category the participant belonged to and gave the participant the date and time of a focus group with other mothers of a similar acculturation level.

The focus groups were approved by the Cal Poly Human Subjects Committee. All participants provided written informed consent in Spanish or English (Appendix K). All forms were written in English, translated into Spanish and then back-translated into English to ensure accuracy. These forms included the study explanation form, interest form and short demographic form given at the time of recruitment and the longer demographic form (Appendix M) and informed consent form given during the focus groups.

Almost all recruits were either low acculturated or bi acculturated so 9 focus groups were conducted: 4 bi-acculturated focus groups, 4 low acculturated focus groups and 1 high acculturated focus group. High acculturated mothers who did not attend the scheduled focus group were later called back for one on one interviews. Three of the ten mothers called were interviewed. WIC focus groups $(n=12)$ took place at the WIC clinic in Grover Beach. Head Start focus groups $(\mathrm{n}=21)$ were held in Head Start classrooms in Paso Robles and Five Cities. The focus groups were conducted in Spanish for the low and bi acculturated mothers and in English for the high acculturated group. Each focus group lasted between one hour-and-a-half to two hours and was lead by trained moderator who was a bilingual member of the community. In addition there was a bilingual note-taker and a bilingual facilitator at each focus group. All sessions were audio-tape recorded. 
Training for the focus group facilitator and note takers was conducted. Also, to ensure our focus group questions were acceptable, two "mock" focus groups were done with about 5 volunteers from the community each time. The wording of questions was changed and the word "Americanized" was chosen to replace "Acculturated" in order to enhance comprehensibility.

The focus groups all used the following format:

Mingling time: 10 to 15 minutes for mothers to arrive, eat some snacks and talk. During this time, note takers obtained written and verbal informed consent.

Introduction: Each individual in the room introduced themselves using their first name only. Participants were informed of audio-recording of the session.

Explanation: The research was explained by the focus group moderator, using a script to assure accuracy and consistency. It included a description of acculturation.

Guidelines were explained as follows:

1. There are no incorrect answers, only different points of view.

2. One person should talk at a time for courtesy and to allow for proper recording.

3. It is ok to disagree with others but we ask that each person listen respectfully.

4. The moderator will guide the discussion.

5. Cell phones should be turned off.

The main questions were centered around the effect of acculturation on child feeding practices and maternal perceptions of a healthy body size (Appendix N). At the end of the focus group session the note takers summarized what they had heard the mothers discuss and participants were given the opportunity to add additional comments. 
After the focus groups the note-takers typed up their notes and if written originally in Spanish, translated them into English. They also transcribed the audio recordings, translating them into English as needed.

Transcriptions from each focus group were then analyzed for themes in a systematic manner. All of the transcripts from the focus groups from the same level of acculturation were first read completely to gain an understanding of the focus group dynamics. Then, each focus group transcript from the same level of acculturation was read individually. In order to analyze the focus group data, each of the focus group questions were written on a blank document. On the document under each question, each new idea was recorded as a theme. Each time a person spoke, their ideas were recorded and a tally was set next to each of the themes to keep track of the total number of comments as well as how often one theme or idea was mentioned. The tallies were also labeled with a code so that we knew in which focus group the theme or idea was mentioned. The codes were as follows: "FC Low" for the low acculturated group who met in Five Cities; "Paso Low" for the low acculturated group who met in Paso Robles; "WIC Low" 1 and 2 for the first and second, respectively low acculturated group that met at WIC. The Bi acculturated groups were given similar codes except "Low" was replaced with "Bi". After this was complete, this author reviewed the set of themes and organized them into major themes and sub themes. Here is an example of an analysis from the focus groups:

1. The question: Ok, now I'd like to know what are the differences in the way your mother fed you compared to how you now feed your children?

\section{Responses:}


- “Ok, the difference when we were children was that they didn't give us too much food. At least in my case. Since we weren't very high class, regarding how much my father earned, it barely was enough for the week. We ate meat every $3^{\text {rd }}$ day and only a little bit and they didn't serve us how we serve our children like big plates well served with more food while back home (in Mexico) everybody had their ration and we didn't get seconds."

- "In my case, similar to hers, but we had meat every 8 days, every Sunday or sometimes not even Sundays. Mostly beans...that's just how we grew up. We're 12 siblings, lots of family and little work so the portions were also small. It wasn't very much food like now. We give the abundance to our children."

\section{Recording of the themes:}

Ok, now I'd like to know what are the differences in the way your mother fed you compared to how you now feed your children?

\section{They had less food in Mexico than they do in the U.S. (FC Low: //)}

Subthemes: They barely had enough food (FC Low: /)

Less meat (FC Low: //)

Smaller portions (FC Low: /)

Now they feed their children much more (FC Low: //)

After each focus group from the low acculturated group had been read for themes and comments had been tallied, the total number of comments from each question were added together. The frequency of comments related to each theme was calculated in order to determine the percentage of time one theme was stated from the total comments 
directed towards the question that was being analyzed. The bi acculturated groups and then the high acculturated group were analyzed in the same manner.

Sometimes the mothers would get off topic when answering a question and unintentionally answer one of the previous questions we had asked in the focus group. If this were the case, those comments and ideas were recorded under the question they most closely related too. If a comment was completely off topic and did not answer any of the questions posed, we disregarded it. 


\section{CHAPTER 4}

\section{RESULTS}

\subsection{Aim 1}

\subsubsection{Objective 1A: Demographics and Anthropometrics}

One hundred-and-nine women-child dyads were recruited, 25 pairs dropped out (22.9\%), leaving 84 women in the original study. Of the 84 mother-child dyads 47 had children from 0-18 months old and 37 from 19-36 months old. Mothers were later called back in order to obtain acculturation data, but 16 of the mothers called were unavailable and were therefore excluded from the data analysis. In total there were 68 participants included in Aim 1. The mean age of the 68 participants from Aim 1 was 28.4 years, ranging from 16 to 45 years, and their children's average age was 15 months, ranging from 2.3 months to 32.8 months. The average age of a child in the low acculturated group was 16.2 month and the average age of a child in the high acculturated group was 12.7 months. A little over half of participants (55.9\%) were unemployed. A majority of participants were married and had an average of 4.3 persons residing in their home. The average years of education was 9.7 years and the average family income was $\$ 17,434$ per year. These descriptive factors were no different between the participants in which there was enough information to determine acculturation $(n=68)$ and the group unable to be used $(n=16)$ in the acculturation comparisons. The full demographics information for all the 84 dyads can be found in Table 4-1.

Of the factors measured by acculturation status, there was a difference in family size, income (which may be related to increased family size), maternal education level and maternal age (Table 4-2). The low acculturated mothers on average earned more than 
the high acculturated, had a larger family size, a lower level of education and were older.

On average the low acculturated mothers had a BMI of $29.8 \mathrm{~kg} / \mathrm{m}^{2}$ and the high

acculturated had a BMI of $29.9 \mathrm{~kg} / \mathrm{m}^{2}$, these were not statistically significantly different.

Table 4-1 Demographics of WIC mother-child dyads. (Aim 1).

\begin{tabular}{|c|c|c|c|}
\hline & $\begin{array}{l}\text { Mothers with } \\
\text { acculturation data } \\
n=68(\%)\end{array}$ & $\begin{array}{l}\text { Mothers without } \\
\text { acculturation data } \\
n=16(\%)\end{array}$ & P-values \\
\hline Employment Status & & & .185 \\
\hline Employed, \% & $30 \quad(44.1)$ & $10 \quad(62.5)$ & \\
\hline Unemployed, \% & $38 \quad(55.9)$ & $(37.5)$ & \\
\hline Marital Status & & & .228 \\
\hline Single & $23 \quad(33.8)$ & $(50.0)$ & \\
\hline \multirow[t]{2}{*}{ Married } & $45 \quad(66.2)$ & $8 \quad(50.0)$ & \\
\hline & Mean (SD) & Mean (SD) & T test $p$ value \\
\hline Income (dollars) & $17,433.5(756.49)$ & $17,367.5(696.47)$ & .974 \\
\hline Family Size (total people) & $4.368(1.27)$ & $3.938(1.24)$ & .191 \\
\hline Education (highest grade) & $9.676(2.98)$ & $10.313(2.73)$ & .418 \\
\hline Mother's Age (years) & $28.4(5.96)$ & $26.4(4.98)$ & .167 \\
\hline Child's Age (months) & $15.0(9.4)$ & $20.4(7.8)$ & $.026 *$ \\
\hline
\end{tabular}


Table 4-2 Demographic factors by acculturation status (Aim 1).

\begin{tabular}{|c|c|c|c|}
\hline & $\begin{array}{l}\text { Low } \\
\text { Acculturated } \\
\text { Mothers (percent) } \\
n=\mathbf{4 5}(\%)\end{array}$ & $\begin{array}{l}\text { High } \\
\text { Acculturated } \\
\text { Mothers } \\
\text { (percent) } \\
\mathbf{n}=\mathbf{2 3}(\%)\end{array}$ & P-values \\
\hline Employment Status & & & .940 \\
\hline Employed & $(44.4)$ & $(43.5)$ & \\
\hline Unemployed & $(55.6)$ & $(56.5)$ & \\
\hline Marital Status & & & .081 \\
\hline Single & $(26.7)$ & $(33.8)$ & \\
\hline \multirow{2}{*}{ Married } & $33 \quad(73.3)$ & $12 \quad(52.2)$ & \\
\hline & $\begin{array}{l}\text { Low Acculturated } \\
\text { mean (SD) }\end{array}$ & $\begin{array}{l}\text { High } \\
\text { Acculturated } \\
\text { mean (SD) }\end{array}$ & T test $p$-value \\
\hline Income (dollars) & $19,454(624.1)$ & $13,483(832.2)$ & $0.005^{*}$ \\
\hline Family Size (total people) & $4.8(1.1)$ & $3.5(1.1)$ & $0.001 *$ \\
\hline Education (highest grade) & $8.4(2.7)$ & $12.3(1.5)$ & $0.000 *$ \\
\hline Mother's Age (years) & $30.6(5.4)$ & $24.1(5.3)$ & $0.000 *$ \\
\hline BMI $\left(\mathrm{kg} / \mathrm{m}^{2}\right)$ & $29.8(5.9)$ & $29.9(6.8)$ & $0.637 \dagger$ \\
\hline Child's Age (months) & $16.2(10.1)$ & $12.7(7.3)$ & 0.105 \\
\hline
\end{tabular}

\subsubsection{Objective 1B: Food Frequency}

Thirteen children most of whom were under 6 months of age were not included in this analysis because they were not consuming solids yet leaving 55 children in this analysis. Children on average consumed 3 of the "healthy food items" each day and 1 of the "less healthy" food items each day (data not shown). Fruit was the most common healthy food item consumed. The three most commonly consumed less healthy food items were sweets, sugary cereals and sweetened drinks. Thirty-six percent of children consumed less than 1 vegetable a day, and $34 \%$ consumed less than 1 fruit a day. Children consumed an average of 9.3 servings of vegetables per week, 10.4 servings of fruits per week and 4.2 servings of legumes per week. These did not appear to be 
different between the acculturation levels of the mothers except for bean consumption as shown in Table 4-3. The children of the low acculturated mothers were eating an average of 5.2 servings of legumes a week compared with the children of high acculturated mothers who consumed an average of 2.1 servings of legumes per week.

Table 4-3 Average weekly number of servings consumed of each of the food items used to make up our composite food scores for total participants, and comparing low acculturated and high acculturated participants.

\begin{tabular}{ll|llc}
\hline Food Item & $\begin{array}{l}\text { Average weekly } \\
\text { intake for all } \\
\text { participants } \\
\mathbf{n = 5 5} \text { (SD) }\end{array}$ & $\begin{array}{l}\text { Average weekly } \\
\text { intake for low } \\
\text { acculturated } \\
\mathbf{n = 3 7} \text { (SD) }\end{array}$ & $\begin{array}{l}\text { Average } \\
\text { weekly intake } \\
\text { for high } \\
\text { acculturated } \\
\text { n=18 (SD) }\end{array}$ & P-value \\
\hline Sweet Drinks & $1.3(2.3)$ & $0.6(2.3)$ & $1.0(1.6)$ & 0.233 \\
Soda & $0.8(1.8)$ & $0.7(1.6)$ & $0.6(1.7)$ & 0.761 \\
Sugary Cereal & $2.0(3.5)$ & $2.0(3.8)$ & $1.4(2.6)$ & 0.484 \\
Fast Food & $0.7(1.0)$ & $0.8(1.1)$ & $0.6(0.5)$ & 0.419 \\
Sweets & $2.6(4.8)$ & $2.0(3.9)$ & $2.7(5.2)$ & 0.640 \\
French Fries & $0.8(1.0)$ & $0.8(1.1)$ & $0.6(0.5)$ & 0.421 \\
Any Vegetable & $9.3(6.8)$ & $10.1(7.1)$ & $7.9(6.5)$ & 0.267 \\
Any Fruit & $10.4(7.1)$ & $10.5(7.6)$ & $9.7(6.5)$ & 0.673 \\
Beans & $4.2(5.1)$ & $5.2(5.6)$ & $2.1(2.8)$ & $0.006^{*}$
\end{tabular}

$\mathrm{T}$ tests were used to measure the differences in food consumption between acculturation level.

To determine the effect of several variables on the frequency of consumption of "less healthy" and "healthy" foods, continuous variables of child's age in months, mother's age in years, mother's BMI, mothers years of education and the categorical variable of mother's acculturation level were used in the model. In the unadjusted analysis (found in Appendix E) only child's age had significant positive effect on the number of both "less healthy" and "healthy" food items consumed. Acculturation had a slight non-significant effect on food frequency of healthy foods; with an increase in acculturation there was a decrease in healthy foods (p-value of 0.073). 
In the adjusted analysis of food groups (Table 4-4), only child's age had a significant effect on amount of "less healthy" and "healthy" foods consumed. With each month increase of age there was an increase of 0.12 servings of "less healthy" foods and an increase of 0.07 of the "healthy" foods.

In the adjusted analysis for healthy foods mother's BMI was the other variable with significant effects. As the mother's BMI increased, there was a decrease in healthy items consumed by the child. There was no difference in effect by acculturation level on intake of "less healthy" or "healthy" food intake.

Table 4-4 Unhealthy and healthy food's relationship by acculturation, child age, mother's age, mother's BMI and mother's education status, adjusted results.

\begin{tabular}{lll|ll}
\hline & $\begin{array}{l}\text { Less } \\
\text { healthy } \\
\text { foods } \\
\text { estimated } \\
\text { coefficient }\end{array}$ & $\begin{array}{l}\text { n=55 } \\
\text { P-Value }\end{array}$ & $\begin{array}{l}\text { Healthy } \\
\text { foods } \\
\text { estimated } \\
\text { coefficient }\end{array}$ & $\begin{array}{l}\text { n = 55 } \\
\text { P-Value }\end{array}$ \\
\hline $\begin{array}{l}\text { Acculturation } \\
\text { (High Versus }\end{array}$ & -0.090 & 0.710 & -0.173 & 0.557 \\
$\begin{array}{l}\text { Low) } \\
\text { Child Age } \\
\text { (months) }\end{array}$ & 0.122 & $0.000^{*}$ & 0.066 & $0.011^{*}$ \\
$\begin{array}{l}\text { Mother Age } \\
\text { (years) }\end{array}$ & -0.001 & 0.966 & -0.040 & 0.320 \\
$\begin{array}{l}\text { Mother BMI } \\
\left.\text { (m/kg }{ }^{2}\right)\end{array}$ & 0.012 & 0.460 & -0.042 & $0.035^{*}$ \\
$\begin{array}{l}\text { Years } \\
\text { Education }\end{array}$ & -0.000 & 0.994 & -0.038 & 0.663 \\
\hline Geable & & & &
\end{tabular}

General linear regression model using square root of food items.

\subsubsection{Objective 1Ca: Maternal Perceptions of Adult Body Size}

On average, the mother's tended to underestimate their own body size by a score of -1.5 (SD 1.6). About $10 \%$ of women accurately perceived their own weight. This means that in the picture scales they chose a picture for themselves that was between one and two figures smaller than the figure that accurately depicted their BMI. This 
underestimation would be equivalent to approximately 5.5 BMI units. The unadjusted analysis of mother's perceptions of a typical, healthy and preferred female body size and the image that she believed to best represent her own body size can be found in Table 45. For each of the questions, in general the high acculturated group chose figures that were larger than the low acculturated group, however, only the figures chosen for a typical Hispanic woman and for a healthy woman were statically significantly different. For the typical Hispanic female both groups chose a figure score associated with an overweight woman. The high acculturated women however, chose a higher score on average (5.8 \pm 1.3$)$ for the typical Hispanic woman than did the low acculturated women $(5.0 \pm 1.4)$. For the healthy sized woman, the high acculturated women chose a higher score on average ( 4.0 with a SD of 0.7$)$ than did the low acculturated women ( 3.5 with a SD of 0.9). Both of these estimations were consistent with a BMI in the normal range $\left(18.5-24.9 \mathrm{~kg} / \mathrm{m}^{2}\right)$.

In order to determine the effect of acculturation on mother's accuracy in perceiving her own body size the effect of multiple variables on the accuracy of her perception included the categorical variable of acculturation, and continuous variables of child's age in months, mother's age in years, mother's BMI, and mothers education in years. In the unadjusted analysis (found in Appendix E) only mother's age and her BMI had significant negative effects on mother's perception of self, meaning as mothers age or BMI increased, her accuracy decreased. Acculturation had a positive directional effect but was not found to be significant $(\mathrm{p} \leq 0.058)$, meaning that as acculturation level increased accuracy also increased. Once all of the variables were controlled for (Table 46) maternal age no longer had an effect but child's age did and the effect of mother's 
BMI remained. As child's age increased mothers became more accurate in estimating their own weight $(\mathrm{p} \leq 0.043)$ and as mother's BMI increased the mothers were more likely to underestimate their own weight $(\mathrm{p} \leq .0 .011)$. There was a trend toward a relationship between acculturation and maternal perceptions of her own body weight, such that as the mothers became more acculturated they were more likely to accurately perceive their own body weight but this did not reach significance $(\mathrm{P} \leq 0.058)$.

Table 4-5 Maternal perceptions of women scores, an unadjusted analysis.

\begin{tabular}{l|l|l|l} 
& $\begin{array}{l}\text { Mean Low } \\
\text { Acculturated } \\
\text { Score (SD) }\end{array}$ & $\begin{array}{l}\text { Mean High } \\
\text { Acculturated } \\
\text { Score (SD) }\end{array}$ & P-Value \\
\hline Typical Woman & $5.0(1.4)$ & $5.8(1.3)$ & $0.021^{*}$ \\
Healthy Woman & $3.5(0.9)$ & $4.0(0.7)$ & $0.016^{*}$ \\
Preferred Woman & $3.2(0.8)$ & $3.6(0.9)$ & 0.169 \\
Represents you & $4.2(1.4)$ & $4.5(1.6)$ & 0.375
\end{tabular}

T-tests were used to compare variables between levels of acculturation.

Table 4-6 Mother's actual versus perceived perception of her self and her child and it's relationship between acculturation, child age, mother's age, mother's BMI and mother's education status: adjusted.

\begin{tabular}{lll|ll}
\hline Variable & $\begin{array}{l}\text { Coefficient } \\
\text { (Perception } \\
\text { of self) }\end{array}$ & $\begin{array}{l}\text { n=68 } \\
\text { P-Value }\end{array}$ & $\begin{array}{l}\text { Coefficient } \\
\text { (Perception } \\
\text { of child) }\end{array}$ & $\begin{array}{l}\text { n=68 } \\
\text { P-Value }\end{array}$ \\
\hline Acculturation & 0.516 & 0.058 & 0.266 & 0.218 \\
Child Age & 0.041 & $0.043^{*}$ & -0.016 & 0.314 \\
Mother Age & -0.038 & 0.295 & -0.013 & 0.646 \\
$\begin{array}{l}\text { Education } \\
\text { Status }\end{array}$ & -0.071 & 0.376 & -0.020 & 0.749 \\
$\begin{array}{l}\text { Mother BMI } \\
\text { General Linear Regression model was fit. }\end{array}$ & -0.080 & \\
\end{tabular}




\subsubsection{Objective 1Cb: Maternal Perceptions of Child's Body Size}

On average, mothers underestimated their child/infant's body size by a figure score of about $-1.1(\mathrm{SD} \pm 1.2)$. Mothers correctly assessed their child's body size $19.3 \%$ of the time. The unadjusted analysis of mother's perceptions of a typical, healthy and preferred infant and child and the image that represented her own infant or child can be found in Tables 4-7 and 4-8. For each of the 4 questions for both the infants and the children, the results were similar to those found with adult women's body size. In general, the high acculturated mothers chose larger figures than the low acculturated mothers did. Of the 4 questions asked about the mother's perceptions of child's body size, there was a significant difference by acculturation level with the mother's view of a typical Hispanic child or infant in her neighborhood and a mother's view of a healthy child or infant. When asked which infant the mother thought looked like a typical infant in her neighborhood the high acculturated chose an average score of 3.7 (SD 0.7) and the low acculturated mother chose a score of 3.1 (SD 1.1). Both of these scores correlate with an infant within a healthy height for weight percentile. When asked which infant the mother thought looked like a healthy infant the high acculturated chose an average score of 3.0 (SD 0.7) and the low acculturated mother chose a score of 2.5 (SD 0.9). The average score for the low acculturated was on the low end of healthy. Results for the toddler's score were very similar to those of the infants and can be found in Table 4-8. The only main difference between the toddler's scores and the infant's scores was that the preferred child's size was higher for the high acculturated mothers (2.9 SD 0.1) than the low acculturated mothers (2.5 SD 0.7). These findings had a directional relationship but were not considered statistically significantly different $(\mathrm{p} \leq 0.056)$. 
In order to determine the effect of acculturation on mother's accuracy in perceiving her child's body size multiple variables including the categorical variable of mother's acculturation, and the continuous variables of child's age in months, mother's age in years, mother's BMI, and mothers education in years were used in the model. In the unadjusted analysis (found in Appendix E Tables AK-2,3) mother's age and mother's BMI had significant negative effects on mother's perception of her child's body size. Once all of the variables were controlled for in a multivariate model (Table 4-6) only maternal BMI had a significant effect on maternal perceptions of her child. As mother's BMI increased she became more likely to underestimate her child's actual body size.

Table 4-7 Maternal perceptions of infant scores aged 0-12 months old, an unadjusted analysis.

\begin{tabular}{l|l|l|l} 
& $\begin{array}{l}\text { Mean Low } \\
\text { Acculturated } \\
\text { Score (SD) }\end{array}$ & $\begin{array}{l}\text { Mean High } \\
\text { Acculturated } \\
\text { Score (SD) }\end{array}$ & P-Value \\
\hline Typical infant & $3.1(1.1)$ & $3.7(0.7)$ & $0.017^{*}$ \\
Healthy infant & $2.5(0.9)$ & $3.0(0.7)$ & $0.007^{*}$ \\
Preferred infant & $2.7(0.9)$ & $3.1(0.9)$ & 0.106 \\
Represents your & $2.6(1.0)$ & $2.9(0.9)$ & 0.306 \\
infant & & &
\end{tabular}

T-tests were used to compare variables between levels of acculturation.

Table 4-8 Maternal perceptions of child scores aged 1-2 years old, an unadjusted analysis.

\begin{tabular}{l|l|l|l} 
& $\begin{array}{l}\text { Mean Low } \\
\text { Acculturated } \\
\text { Score (SD) }\end{array}$ & $\begin{array}{l}\text { Mean High } \\
\text { Acculturated } \\
\text { Score (SD) }\end{array}$ & \\
\hline P-Value \\
\hline Typical Child & $2.7(1.1)$ & $3.4(0.8)$ & $0.003^{*}$ \\
Healthy Child & $2.3(0.8)$ & $2.7(0.6)$ & $0.016^{*}$ \\
Preferred Child & $2.5(0.7)$ & $2.9(0.7)$ & 0.056 \\
$\begin{array}{l}\text { Represents your } \\
\text { child }\end{array}$ & $2.3(1.0)$ & $2.7(0.1)$ & 0.137
\end{tabular}

T-tests were used to compare variables between levels of acculturation. 


\subsubsection{Objective 1D: Parenting Stress Levels}

In general, the participant's chose answers that indicated low parenting stress.

Answers ranged from 1 to 4 with a 1 indicating the lowest level of parenting stress and a 4 indicating the highest level of parenting stress. The average answer choice for child related stress questions was 1.4 and the average answer for the mother related stress questions was 1.5 . Only $1(1.8 \%)$ participant had an average score of 3 or higher on both the child related stress score and the parent related stress score. In order to determine the effect of acculturation on parenting stress the effect of individual variables described in the previous analysis were used. In the unadjusted univariate model analysis (Appendix E. Table AK-3) none of the variables had a significant effect on either the parent related stress score or the child derived stress score. After adjusting for each of the variables (Table 4-9), the results remained the same. Education status did show a small but nonsignificant effect on parent related stress scores. As education increased, the parent stress score tended to decrease $(\mathrm{p} \leq 0.072)$.

Table 4-9 Parenting stress score by subscale: parent related and child related stress and their relationship between acculturation, child age, mother's age, mother's BMI and mother's education status: adjusted.

\begin{tabular}{lll|ll} 
& $\begin{array}{l}\text { Parent related } \\
\text { Score } \\
\text { Variable }\end{array}$ & $\begin{array}{l}\text { Coefficient } \\
\text { n=68 }\end{array}$ & $\begin{array}{l}\text { P-Value } \\
\text { score } \\
\text { coefficient }\end{array}$ & $\begin{array}{l}\text { (-68 } \\
\text { P-Value }\end{array}$ \\
\hline Acculturation & 0.897 & 0.373 & -0.312 & 0.756 \\
Child Age & 0.267 & 0.790 & 0.505 & 0.615 \\
Mother Age & 0.183 & 0.855 & -0.760 & 0.450 \\
$\begin{array}{l}\text { Education } \\
\text { Status }\end{array}$ & -1.828 & 0.072 & -1.243 & 0.218 \\
Mother BMI & -0.687 & & & \\
\hline
\end{tabular}

General Linear Regression model was fit. 


\subsection{Aim 2: Key Informant Interviews}

Key informant interviews were used to help understand the results from the above data analysis and to develop focus group discussion questions. A total of 9 key informant interviews were conducted. There were two important themes identified through the interviews that were related to dietary acculturation. One theme was that mothers who are more acculturated will give their children more westernized foods $(55 \%$ of interviewees reported this) including fast foods, sports drinks, and sugary cereals while mothers with lower acculturation levels prefer their "native" foods $(66.7 \%$ of interviewees reported this). One interviewee stated "They're buying into more fast food and highly refined processed foods, sugary drinks. And the mothers with lower acculturation are still gravitating towards their native foods." A second theme that was mentioned by $55 \%$ of interviewees was that exposure, convenience and a desire to fit in are what lead to dietary acculturation. A quote representing this theme include: "but their children who attend school and are much more intermixed with people who have been here for a long time are going to want to be like their peers."

The main theme on maternal perceptions of their child's body size was that Hispanics, regardless of acculturation level, believe that a chubby baby is a healthy baby (33\% of participants): “...Hispanics think that chubby kids are the healthiest”. A complete list of themes and quotes can be found in Appendix $\mathrm{H}$. 


\subsection{Aim 3: Focus Groups}

\subsubsection{Objective 1: Demographics}

A total of 96 women were recruited for our low and bi acculturated focus groups, 51 from WIC and 45 from Head Start. The drop out rate was high with $78.4 \%$ of WIC recruits and $55 \%$ of Head Start recruits not attending the focus groups. SLO county WIC reports a regular 50\% re-appointment rate so the "no show" rate was higher than expected. We had a $65.6 \%$ attrition rate overall with 63 women who dropped out and 32 who participated; 11 of the drop outs were from WIC and 21 were from Head Start. The main reason noted for being unable to participate was a conflict between the time of the focus group and the mother's work schedule. Each focus group contained 2-7 individuals with an average of 3.25 participants for each of the high acculturated focus groups and an average of 4.25 participants for each of the low acculturated focus groups.

Approximately $58 \%$ of our participants were unemployed, most were between the ages of 25 and 34 and almost half had two children. A majority were married (74.2\%) and born in Mexico (94.6\%). Participants were no different from the drop outs in their employment status, number of children, age and acculturation status but were more likely to be born in Mexico and married. This suggests it was more common for a mother to be absent from our focus groups if she was born in the U.S. and was single. A full table of comparative demographics can be found in Table 4-10. 
Table 4-10 Focus group demographics in focus group participants and those who dropped out. Aim 3.

\begin{tabular}{ll|ll}
\hline & $\begin{array}{l}\text { Participated } \\
\mathbf{n}=\mathbf{3 1}(\%)\end{array}$ & $\begin{array}{l}\text { Drop outs } \\
\mathbf{n = 6 3} \text { (\%) }\end{array}$ & $\begin{array}{l}\text { P- } \\
\text { value }\end{array}$ \\
\hline Work Status: & & & \\
\hline Unemployed & $18(58.1)$ & $36(57.1)$ & 0.932 \\
Employed & $13(41.9)$ & $27(42.9)$ & \\
\hline Origin: & & & \\
\hline U.S. & $2(6.5)$ & $13(20.6)$ & 0.060 \\
Mexico & $29(93.6)$ & $50(79.4)$ & \\
\hline Marital Status: & & & \\
\hline Single & $8(25.8)$ & $29(46.0)$ & 0.059 \\
Married & $23(74.2)$ & $34(54.0)$ & \\
\hline Number of & & & \\
Children: & $4(12.9)$ & $16(25.4)$ & 0.368 \\
\hline 1 & $15(48.4)$ & $20(31.8)$ & \\
2 & $8(25.8)$ & $18(28.6)$ & \\
3 & $4(12.9)$ & $9(14.3)$ & \\
$4+$ & & & \\
\hline Age: & $0(0)$ & $5(7.9)$ & 0.311 \\
\hline $16-19$ & $5(16.1)$ & $17(27.0)$ & \\
$20-24$ & $10(32.3)$ & $18(28.6)$ & \\
$25-29$ & $10(32.3)$ & $15(23.8)$ & \\
$30-34$ & $6(19.4)$ & $8(12.7)$ & \\
$35+$ & & & \\
\hline Acculturation: & & & \\
\hline Bi-Acculturated & $13(40.6)$ & $32(50.8)$ & 0.348 \\
Low-Acculturated & $19(59.4)$ & $31(49.2)$ & \\
\hline
\end{tabular}

\subsubsection{Objective 2: Focus Group Themes}

Common themes across all low acculturated and bi acculturated focus groups.

There were many themes found during the focus groups and many of these themes were similar across both levels of acculturation. A full table of themes can be found in the Appendix O but Tables 4-11 to 4-20 contain some of the most predominant themes. Both groups of mothers mentioned that they try to cook the same way their mothers had cooked for them but that cooking traditional foods can be difficult due to the different taste of American ingredients and their children's preferences for American foods. One 
mother states: "Now with my children, the food is not the same because my mom had her own flavoring. I have never been able to cook well, so I've tried to make them the same things but the difference is that the meats from here even have a different flavoring. Even if I try to make them the same way, the flavoring is not the same as how they used to cook them. My mom had good flavoring, but I lost it (Paso Low Acculturated)". Both groups also stated they had made some changes to their diet; mainly they try to incorporate healthy foods and that they make some American dishes. In both of the groups a majority of the comments about priorities when grocery shopping were about health. Both groups agreed that more acculturated mothers tend to cook more convenience foods and look at nutrition labels when grocery shopping and that less Americanized mothers think less about health when it comes to eating or shopping for food.

When discussing how these mothers decided if their child is at a healthy weight, a majority in both groups said they get this information from their health care professionals. An example of this can be seen in this Head Start mother's comment: "Well whenever I go to the doctor I ask if they are at a normal weight and height (Paso Low Acculturated)". Some mothers from both groups did not believe the methods of determining a healthy weight for a child (going to the doctor, looking at how clothes fit etc...) would differ between more or less Americanized mothers, however, many comments from both groups showed they believed that less Americanized mothers think a chubby baby is healthy. One woman commented on the culture in Mexico, stating: "there (in Mexico) the culture exists where people say that whoever is chubby is healthy, 
but it's a lie. There were a lot of people who would say that their son was healthy because he was chubby (WIC Bi Acculturated Focus Group \#1)”.

Both groups of mothers stated that feeding infants, especially if you want them to gain or lose weight could be difficult. Both groups also believed that more Americanized mothers are more likely to limit foods given to their children than less Americanized mothers.

\section{Themes that differed between the low and bi acculturation levels}

There were many ways in which the low acculturated and bi acculturated groups differed when talking about food and diet. The low acculturated mothers mentioned having a lot less food when they were growing up in Mexico and that now they feed their children much more than they were fed when they themselves were children. Some of the low acculturated mothers also mentioned that they focused on cravings or on cost when grocery shopping. When discussing the difference between how more Americanized mothers feed their children as compared to less Americanized mothers, the bi acculturated group mentioned that less Americanized mothers are more likely to cook from whole ingredients instead of using processed foods. Throughout the focus groups the bi acculturated group talked about the importance of cooking from whole ingredients, how whole ingredients are good for your health and more affordable, much more frequently than the low acculturated mothers.

The low acculturated mothers mentioned education a lot throughout the focus groups. They mentioned wanting more education when it came to portion sizes and how much their children should weigh. They also mentioned that higher acculturated mothers tend to have more education and that this is due in part to their better English skills. The 
mothers mentioned being afraid to ask doctors questions so they often feel less educated. One mother stated: "And we're too shy to ask (the doctor). I sometimes say "oh, If I knew English I'd ask but sometimes in Spanish, due to fear, one doesn't want to ask" (WIC Low Acculturated \#1).

The low acculturated group also mentioned some of the difficulties they face when trying to feed their children healthier foods. They stated their frustrations with making separate dishes for dinner for each member of the family and with trying to get their children to eat smaller portion sizes.

When talking about how food affects weight, the low acculturated mothers mentioned that genetics might have more of an impact on their children's weight than food. They stated they had family members that eat well and exercise a lot but are still overweight. One mother stated "well, my daughter has been a little big since she was small and I give the same portions to both of my daughters and... I don't understand why one is a bit bigger than the other". The low acculturated groups also stated that parents tend not to notice their child's weight very much. The bi acculturated group believed that less Americanized mothers do not worry about weight very much until they have adapted to the culture of the U.S. They also believe that the media affects their own view as well as their children's view on weight and that their children are seeing thinner images on U.S. television than what the mothers saw in Mexico. One women stated "If you look at previous times on television we would see women that were well formed, they had hips and everything and one would say 'Wow! What a figure!' and now you just see sticks and skinny girls". 
Lastly, when discussing how mothers may be more selective when feeding infants than toddlers, the bi acculturated groups mentioned some of the difficulties in controlling what a toddler eats. They said that toddlers can get their own food and are getting food at school so it is easier to control what an infant eats. The low acculturated mothers mentioned that less Americanized mothers introduce solids earlier to children than more Americanized mothers.

Table 4-11 Focus group themes separated by level of acculturation: differences between how the mothers were fed and how they now feed their children.

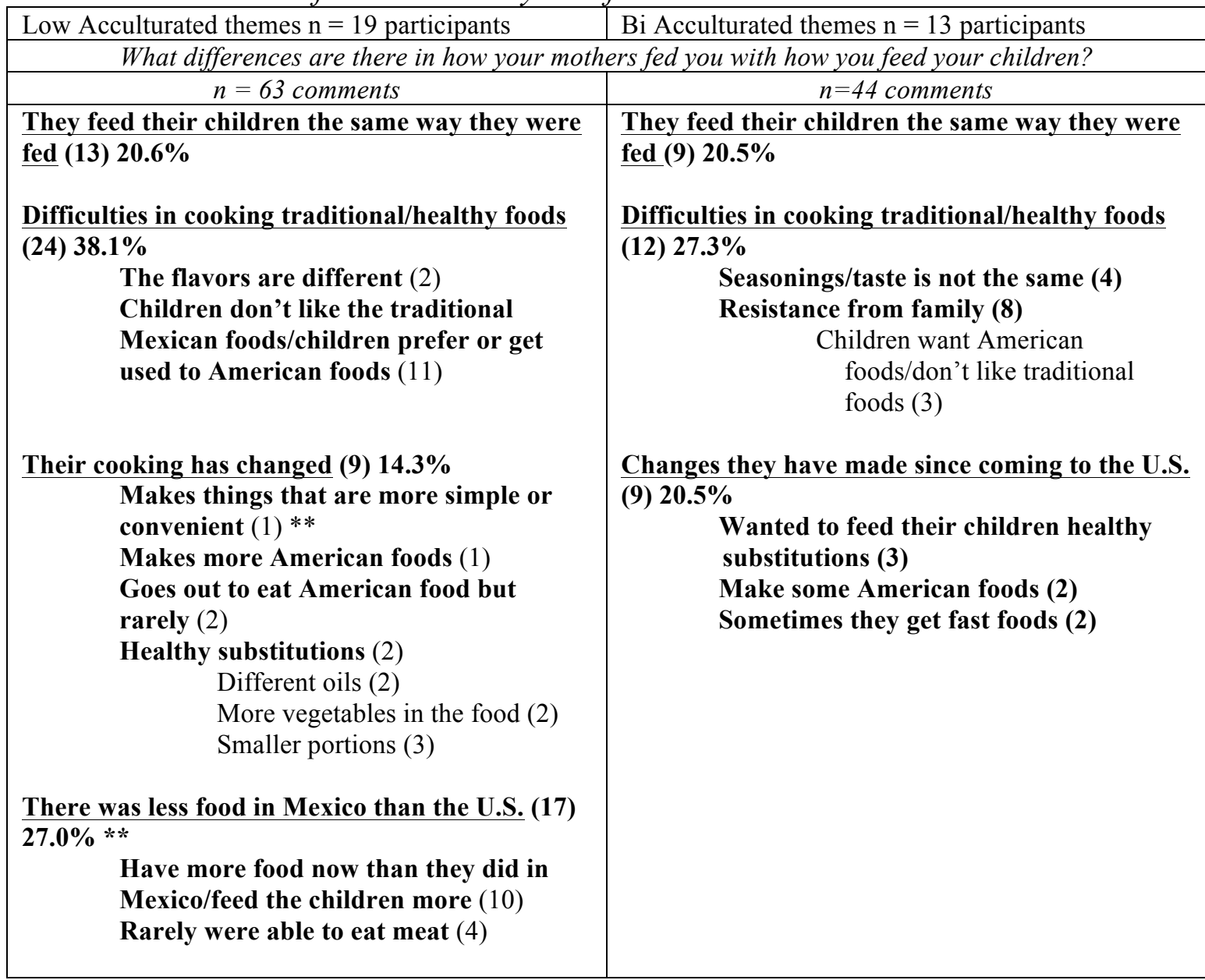

** Refers to a theme that is mentioned in one acculturation group but not the other. Underlined themes have a percentage (\%) of total comments mentioned during the focus groups about the question above them.

Numbers in parenthesis are how often that specific comment was mentioned. 
Table 4-12 Focus group themes separated by level of acculturation: how more or less Americanized mothers differ in how they feed their children.

\begin{tabular}{|c|c|}
\hline Low Acculturated themes $n=19$ participants & Bi Acculturated themes $n=13$ participants \\
\hline \multicolumn{2}{|c|}{$\begin{array}{c}\text { So do you think that moms that are more Americanized feed their children differently compared to Latina } \\
\text { moms that are less Americanized? }\end{array}$} \\
\hline$n=46$ comments & $n=31$ comments \\
\hline $\begin{array}{l}\text { Negative effects of acculturation on diet (19) } \\
\begin{array}{l}\text { 41.3\% } \\
\text { Eat foods that are faster to cook/ } \\
\text { more fast foods }(6)\end{array}\end{array}$ & $\begin{array}{l}\text { Negative changes of Americanization (11) } 35.5 \% \\
\text { More Americanized cook/eat } \\
\text { faster/more processed foods (7) } \\
\text { More Americanized cook more } \\
\text { American foods (2) } * *\end{array}$ \\
\hline Positive effects of acculturation on diet (16) & \\
\hline $\begin{array}{l}34.8 \% * * \\
\text { Eat Smaller portions/limit portions } \\
\text { (4) } \\
\text { Cook with less oil/grease }(4) \\
\text { Some are concerned with health }(10)\end{array}$ & $\begin{array}{l}\text { Cooking from scratch (5) } 16.1 \% * * \\
\text { Less Americanized cook more from } \\
\text { scratch (2) }\end{array}$ \\
\hline
\end{tabular}

** Refers to a theme that is mentioned in one acculturation group but not the other. Underlined themes have a percentage (\%) of total comments mentioned during the focus groups about the question above them.

Numbers in parenthesis are how often that specific comment was mentioned.

Table 4-13 Focus group themes separated by level of acculturation: what is most important in how they choose foods for their children.

\begin{tabular}{|l|l}
\hline Low Acculturated themes $\mathrm{n}=19$ participants & Bi Acculturated themes $\mathrm{n}=13$ participants
\end{tabular}

What is most important to you when you are shopping and choosing food for your children?

\begin{tabular}{|c|c|}
\hline \multicolumn{1}{|c|}{$n=42$ comments } & \multicolumn{1}{c|}{$n=30$ comments } \\
\hline $\begin{array}{c}\text { Health (15) } 35.7 \% \\
\text { Cravings (8) } 19.0 \% * * \\
\text { Only buy junk food as a craving-every } \\
\text { once in a while (2) } \\
\text { Buy what they are craving (2) } \\
\text { Sees junk foods etc as cravings- to eat } \\
\text { aside from your normal meal (3) } \\
\text { Healthy items (12) } \\
\text { Look at labels (2) ** } \\
\text { Cost (6) } 14.3 \% * *\end{array}$ \\
\end{tabular}

** Refers to a theme that is mentioned in one acculturation group but not the other.

Underlined themes have a percentage (\%) of total comments mentioned during the focus groups about the question above them.

Numbers in parenthesis are how often that specific comment was mentioned.

Bold and underlined statements are main themes, bold but not underlined statements are subthemes and statements not in bold describe the subthemes. 
Table 4-14 Focus group themes separated by level of acculturation: differences between more and less Americanized mothers in how they choose foods for their children.

\begin{tabular}{|c|c|}
\hline Low Acculturated themes $n=19$ participants & $\mathrm{Bi}$ Acculturated themes $\mathrm{n}=13$ participants \\
\hline \multicolumn{2}{|c|}{$\begin{array}{l}\text { Do you think that these factors are different for a mother that is more Americanized than for one that is less } \\
\text { Americanized? }\end{array}$} \\
\hline$n=16$ comments & $n=14$ comments \\
\hline $\begin{array}{c}\text { Differences for more Americanized (8) } 50.0 \% \\
\text { Read nutrition labels }(1) \\
\text { Buy convenience foods }(4) * *\end{array}$ & $\begin{array}{l}\text { Differences for more Americanized (3) } 21.4 \% \\
\text { Read labels (1) } \\
\text { More Americanized don't know how to } \\
\text { cook from scratch (1) } * *\end{array}$ \\
\hline $\begin{array}{l}\text { Differences for less Americanized (2) } 12.5 \% \\
\text { Think less about health when buying } \\
\text { foods (1) } \\
\text { Buy what is familiar (1) } * *\end{array}$ & $\frac{\text { Differences for less Americanized (5) } 35.7 \%}{\text { Don't realize the effects of a poor diet (2) }}$ \\
\hline $\begin{array}{l}\text { Do not believe there is a difference between more } \\
\text { and less acculturated mothers (4) } 25.0 \%\end{array}$ & $\begin{array}{l}\text { Do not believe there is a difference between more } \\
\text { and less acculturated mothers (5) } 35.7 \%\end{array}$ \\
\hline
\end{tabular}

** Refers to a theme that is mentioned in one acculturation group but not the other.

Underlined themes have a percentage (\%) of total comments mentioned during the focus groups about the question above them.

Numbers in parenthesis are how often that specific comment was mentioned.

Bold and underlined statements are main themes, bold but not underlined statements are subthemes and statements not in bold describe the subthemes.

Table 4-15 Focus group themes separated by level of acculturation: how a child's diet affects his or her weight.

\begin{tabular}{|c|c|}
\hline Low Acculturated themes $n=19$ participants & Bi Acculturated themes $n=13$ participants \\
\hline \multicolumn{2}{|c|}{ Do you think that what a child eats affects their weight and if so how? } \\
\hline$n=37$ comments & $n=40$ comments \\
\hline Specific foods that contribute to weight gain (10) & Specific foods that contribute to weight gain (20) \\
\hline $27 \%$ & $50.0 \%$ \\
\hline \multicolumn{2}{|l|}{ Food is not the main factor in weight (7) } \\
\hline \multicolumn{2}{|l|}{$18.9 \% * *$} \\
\hline $\begin{array}{l}\text { Their child/family eats a lot and is } \\
\text { still thin }(2)\end{array}$ & \\
\hline Genetics might matter more (5) & \\
\hline Has two children, feeds them the & \\
\hline $\begin{array}{l}\text { same and one is bigger than the } \\
\text { other (1) }\end{array}$ & \\
\hline Family eats well/ exercises but & \\
\hline Had a child who grew into their & \\
\hline weight (1) & \\
\hline
\end{tabular}

** Refers to a theme that is mentioned in one acculturation group but not the other.

Underlined themes have a percentage (\%) of total comments mentioned during the focus groups about the question above them.

Numbers in parenthesis are how often that specific comment was mentioned.

Bold and underlined statements are main themes, bold but not underlined statements are subthemes and statements not in bold describe the subthemes. 
Table 4-16 Focus group themes separated by level of acculturation: how the mothers decide if their child is at a good weight and the difference in this between levels of acculturation.

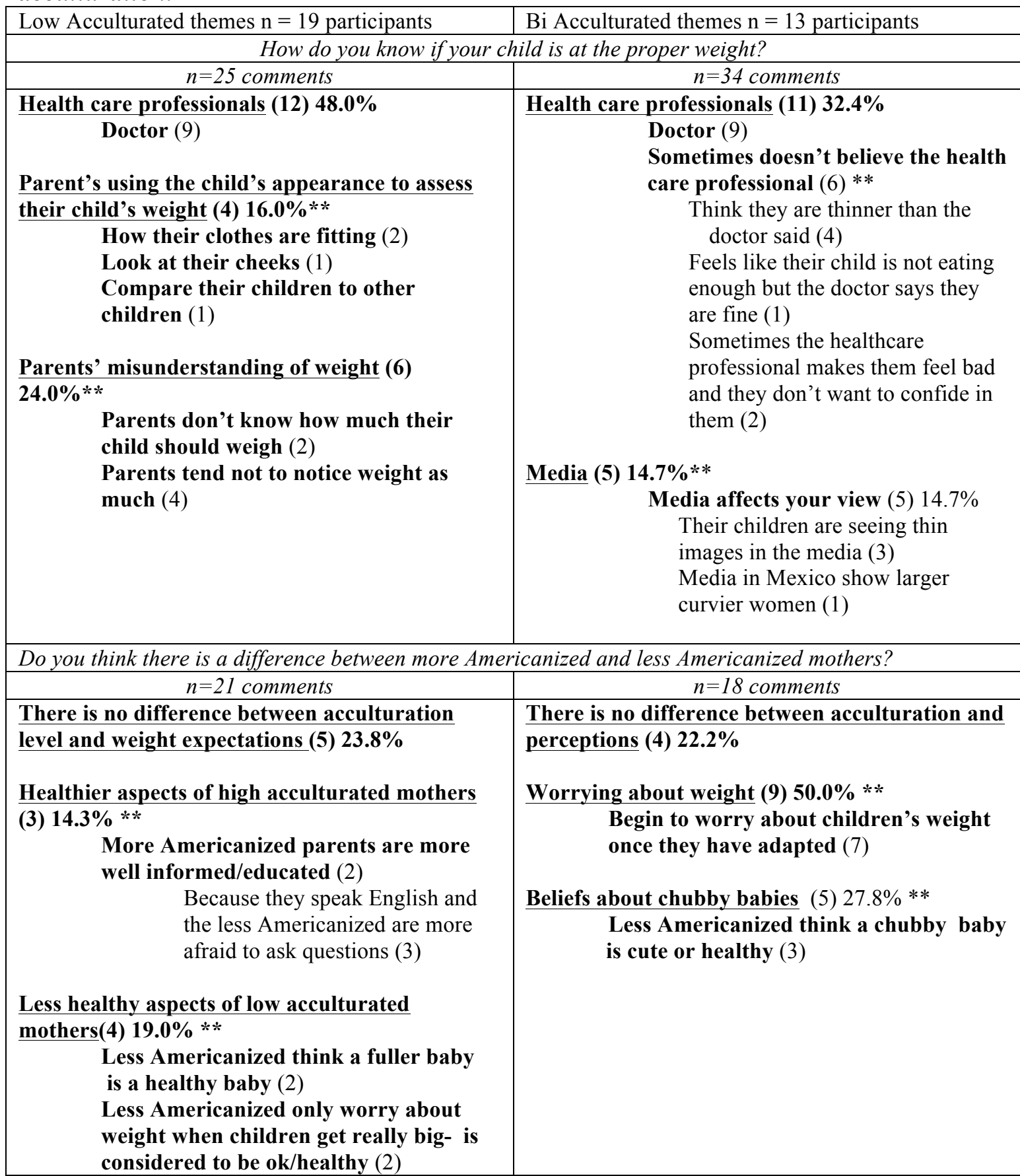

** Refers to a theme that is mentioned in one acculturation group but not the other.

Underlined themes have a percentage (\%) of total comments mentioned during the focus groups about the question above them.

Numbers in parenthesis are how often that specific comment was mentioned.

Bold and underlined statements are main themes, bold but not underlined statements are subthemes and statements not in bold describe the subthemes. 
Table 4-17 Focus group themes separated by level of acculturation: how the mother's view of their child's weight effects what they feed them.

\begin{tabular}{|c|c|}
\hline \multicolumn{1}{|c|}{ Low Acculturated themes $\mathrm{n}=19$ participants } & Bi Acculturated themes $\mathrm{n}=13$ participants \\
\hline \multicolumn{1}{|c|}{ Does the way you see your child's weight affect how you feed them? } \\
\hline $\begin{array}{c}\text { Give overweight children less food (8) } \mathbf{1 5 . 7 \%} \\
\text { Try to feed them less/smaller } \\
\text { portions (7) }\end{array}$ & $\begin{array}{l}\text { Portion Sizes/Amount of foods offered for } \\
\text { overweight kids (5) 19.2\% }\end{array}$ \\
$\begin{array}{c}\text { Difficulties in feeding children well (10) } \mathbf{1 9 . 6 \%} \\
\text { Making different food }\end{array}$ & Increase physical activity (4) $\mathbf{1 5 . 4 \% * *}$ \\
for different people (2) \\
$\begin{array}{l}\text { Laziness (2) } \\
\text { Difficult to give less food to an } \\
\text { overweight child because they are used } \\
\text { to getting a lot of food (3) }\end{array}$ \\
\end{tabular}

** Refers to a theme that is mentioned in one acculturation group but not the other.

Underlined themes have a percentage (\%) of total comments mentioned during the focus groups about the question above them.

Numbers in parenthesis are how often that specific comment was mentioned.

Bold and underlined statements are main themes, bold but not underlined statements are subthemes and statements not in bold describe the subthemes. 
Table 4-18 Focus group themes separated by level of acculturation: how to address overweight and underweight infants.

\begin{tabular}{|c|c|}
\hline Low Acculturated themes $n=19$ participants & Bi Acculturated themes $n=13$ participants \\
\hline \multicolumn{2}{|c|}{ What you would feed an overweight infant? } \\
\hline$n=33$ comments & $n=24$ comments \\
\hline $\begin{array}{c}\text { This is difficult (4) } 12.1 \% \\
\text { This is a difficult age (3) }\end{array}$ & $\begin{array}{l}\text { Feeding infants is difficult (3) } \mathbf{1 2 . 5 \%} \\
\text { They aren't active yet }(1)^{* *} \\
\text { Difficult to keep food from them } \\
(1)^{* *}\end{array}$ \\
\hline $\begin{array}{l}\text { Decrease the amount of food the infant is } \\
\text { receiving (8) } 24.2 \% \\
\text { Smaller portions/ less food (8) }\end{array}$ & $\begin{array}{l}\text { Less Food (10) } 41.7 \% \\
\text { Smaller portions/less food (5) } \\
\text { Listen to your child's body language- } \\
\text { stop feeding when they are full (2) }\end{array}$ \\
\hline $\begin{array}{l}\text { Get education (6) } 18.2 \% * * \\
\text { Get advice from a professional }(4) \\
\text { Feel uneducated of how much is a good } \\
\text { portion to give their child (1) }\end{array}$ & \\
\hline \multicolumn{2}{|c|}{ What would you feed an underweight infant? } \\
\hline$n=11$ comments & $n=14$ comments \\
\hline $\begin{array}{c}\text { Increase amount of food (5) } 45.5 \% \\
\text { Small portions more often (2) }\end{array}$ & $\begin{array}{l}\text { More Food (5) } 35.7 \% \\
\text { Feed them often (3) }\end{array}$ \\
\hline $\begin{array}{l}\text { Offer specific foods (4) } 36.4 \% \\
\text { Meat (1) } \\
\text { Give foods high in fat (1) } * * \\
\text { Whole fat milk (1) } * *\end{array}$ & $\begin{array}{l}\text { Offer specific foods (9) } 64.3 \% \\
\text { Meat }(1) \\
\text { Veggies }(1) * * \\
\text { Iron }(1)^{* *} \\
\text { Vitamins }(1) * *\end{array}$ \\
\hline
\end{tabular}

** Refers to a theme that is mentioned in one acculturation group but not the other.

Underlined themes have a percentage (\%) of total comments mentioned during the focus groups about the question above them.

Numbers in parenthesis are how often that specific comment was mentioned.

Bold and underlined statements are main themes, bold but not underlined statements are subthemes and statements not in bold describe the subthemes. 
Table 4-19 Focus group themes separated by level of acculturation: how to address overweight and underweight toddlers.

\begin{tabular}{|c|c|}
\hline \multicolumn{2}{|c|}{ What would you do for an overweight toddler? } \\
\hline$n=27$ comments & $n=28$ comments \\
\hline $\begin{array}{c}\text { Offer more of specific foods (5) } \mathbf{1 8 . 5 \%} \\
\text { More Vegetables (3) } \\
\text { More Fruit (1) } \\
\text { More water (1) }\end{array}$ & $\begin{array}{c}\text { More of specific foods (10) } 35.7 \% \\
\text { More Veggies (2) } \\
\text { More Water (2) } \\
\text { Soup (1) }\end{array}$ \\
\hline $\begin{array}{l}\text { Offer less of specific foods (7) } \mathbf{2 5 . 9 \%} \\
\text { Less fat (2) } \\
\text { Less Sugar (1) } \\
\text { Less junk food/fast food (2) }\end{array}$ & $\begin{array}{l}\text { Less of specific foods (4) } 14.3 \% \\
\text { Less Carbs (2) } \\
\text { Less Sugar (1) } \\
\text { Less fried foods (1) }\end{array}$ \\
\hline $\begin{array}{l}\text { Offer Less food (5) } 18.5 \% \\
\text { Reduce portion sizes/give less food (5) } \\
\begin{array}{l}\text { Wants more education (3) } 11.1 \% * * \\
\begin{array}{l}\text { Feels like they need more information on } \\
\text { portions for kids }(3)\end{array}\end{array}\end{array}$ & $\begin{array}{l}\text { Amount of food (6) } 21.4 \% \\
\text { Smaller portions }(5)\end{array}$ \\
\hline \multicolumn{2}{|c|}{ What would you feed an underweight toddler? } \\
\hline$n=25$ comments & $n=14$ comments \\
\hline Offer more nutrient dense foods (6) $24.0 \% * *$ & $\frac{\text { Amount of food (4) } 28.6 \% * *}{\text { More food }(3)}$ \\
\hline $\begin{array}{l}\text { Do not want them to become overweight (5) } \\
\begin{array}{l}\text { 20.0\%** } \\
\text { Better to be underweight than } \\
\text { overweight }(4)\end{array}\end{array}$ & $\begin{array}{l}\text { Specific food (8) } 57.1 \% * * \\
\text { Stew with tortillas (3) } \\
\text { Beans (2) } \\
\text { Vitamins (3) }\end{array}$ \\
\hline
\end{tabular}

** Refers to a theme that is mentioned in one acculturation group but not the other.

Underlined themes have a percentage (\%) of total comments mentioned during the focus groups about the question above them.

Numbers in parenthesis are how often that specific comment was mentioned.

Bold and underlined statements are main themes, bold but not underlined statements are subthemes and statements not in bold describe the subthemes. 
Table 4-20 Focus group themes separated by level of acculturation: Selectivity in feeding children and how acculturation affects selectivity.

\begin{tabular}{|c|c|}
\hline Low Acculturated themes $n=19$ participants & Bi Acculturated themes $n=13$ participants \\
\hline \multicolumn{2}{|c|}{$\begin{array}{c}\text { Do you think as mothers you are more selective in choosing or limiting the foods that you feed your infants } \\
\text { compared to a child that is bigger? }\end{array}$} \\
\hline$n=13$ comments & $n=12$ comments \\
\hline $\begin{array}{l}\text { Difficult to compare because different foods are } \\
\text { appropriate depending on the age group (2) } \\
15.4 \%\end{array}$ & $\begin{array}{l}\text { Stages of feeding children (5) } 41.7 \% \\
\text { Can't feed infants and toddlers the } \\
\text { same- infants can't eat all the same } \\
\text { things a toddler can so you limit the } \\
\text { infant more (3) } \\
\text { Difficulties in controlling toddlers (4) } 33.3 \% * * \\
\text { It's easier to control what your infant } \\
\text { eats/more difficult to control your } \\
\text { toddler (4) } \\
\text { Toddlers can get their own food so } \\
\text { its harder to control them (2) } \\
\text { Can't control what your toddler is } \\
\text { getting at school (1) }\end{array}$ \\
\hline \multicolumn{2}{|c|}{ Do you think that this would be the same for a mother who is more or less Americanized? } \\
\hline$n=17$ comments & $n=12$ comments \\
\hline $\begin{array}{l}\text { More Americanized are more likely to limit } \\
\text { foods (4) } 23.5 \% \\
\text { Yes, American culture teaches you to } \\
\text { limit foods. (1) } \\
\text { More Americanized follow the rules } \\
\text { more (1) } \\
\text { More Americanized more } \\
\text { careful/selective in what they feed their } \\
\text { children (2) }\end{array}$ & 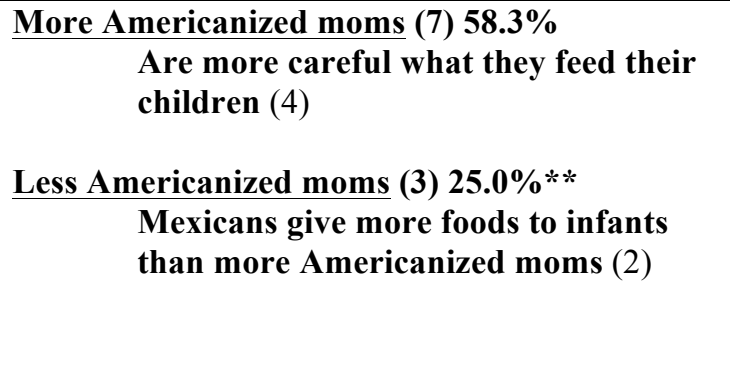 \\
\hline $\begin{array}{l}\text { Less Americanized introduce solids earlier (3) } \\
17.6 \% * *\end{array}$ & \\
\hline $\begin{array}{l}\text { There is no difference between more and less } \\
\text { Americanized (4) } 23.5 \% * *\end{array}$ & \\
\hline
\end{tabular}

** Refers to a theme that is mentioned in one acculturation group but not the other.

Underlined themes have a percentage (\%) of total comments mentioned during the focus groups about the question above them.

Numbers in parenthesis are how often that specific comment was mentioned.

Bold and underlined statements are main themes, bold but not underlined statements are subthemes and statements not in bold describe the subthemes. 


\subsubsection{High acculturated focus groups/interviews: demographics}

The majority of our high acculturated participants were employed, born in the U.S., single and had only 1 child. This was not significantly different between the women who participated and the women who dropped out (Table 4-21), but the small numbers may have limited the ability to detect a difference if there was, in fact, a difference.

Table 4-21 High acculturated focus group and interview demographics, the difference between recruited mothers between mothers who participated and drop outs. Aim 3.

\begin{tabular}{|c|c|c|c|}
\hline & $\begin{array}{l}\text { Participated } \\
\mathrm{n}=5(\%)\end{array}$ & $\begin{array}{l}\text { Drop outs } \\
\mathrm{n}=6(\%)\end{array}$ & $\begin{array}{l}\text { P- } \\
\text { value }\end{array}$ \\
\hline \multicolumn{4}{|l|}{ Work Status: } \\
\hline Unemployed & $1(25.0)$ & $4(66.7)$ & \multirow[t]{2}{*}{0.197} \\
\hline Employed & $3(75.0)$ & $2(33.3)$ & \\
\hline \multicolumn{4}{|l|}{ Origin: } \\
\hline U.S. & $4(100.0)$ & $4(66.7)$ & \multirow[t]{2}{*}{0.197} \\
\hline Mexico & $0(0)$ & $2(33.3)$ & \\
\hline \multicolumn{4}{|c|}{ Marital Status } \\
\hline Single & $4(80.0)$ & $4(66.7)$ & \multirow[t]{2}{*}{0.621} \\
\hline Married & $1(20.0)$ & $2(33.3)$ & \\
\hline \multicolumn{4}{|l|}{$\begin{array}{l}\text { Number of } \\
\text { Children: }\end{array}$} \\
\hline 1 & $3(60.0)$ & $4(66.7)$ & \multirow[t]{2}{*}{0.819} \\
\hline 2 & $2(40.0)$ & $2(33.3)$ & \\
\hline \multicolumn{4}{|l|}{ Age: } \\
\hline $16-24$ & $3(60.0)$ & $\begin{array}{ll}5 & (83.3)\end{array}$ & \multirow[t]{2}{*}{0.387} \\
\hline $25-34$ & $2(40.0)$ & $1(16.7)$ & \\
\hline
\end{tabular}

\subsubsection{High acculturated focus groups: themes}

It was very difficult to identify high acculturated mothers during recruitment. A total of 11 high acculturated mothers were enrolled but only 2 showed up for their appointed focus group. One of the mothers said spaghetti was her favorite dish her mother made for her growing up and the other said Albondigas soup was her favorite. This suggests they may have grown up with a mix of American and Mexican foods. Both of the mothers said they wanted to cook healthier than how their mothers cooked for 
them and they mentioned desiring to eat healthy because of a fear of weight related diseases.

These mothers did believe there would be differences between more Americanized and less Americanized mothers for many of the topics discussed including when choosing what foods to purchase at the grocery store and when deciding if their child was at a healthy weight. Mothers mentioned that the more Americanized mothers receive more education and that the less Americanized mothers are more concerned with taste than health. They also stated that less Americanized mothers do not notice when their child is overweight. A list of the key themes found in the high acculturated mothers is available in Table 4-22. 
Table 4-22 Themes from high acculturated focus group

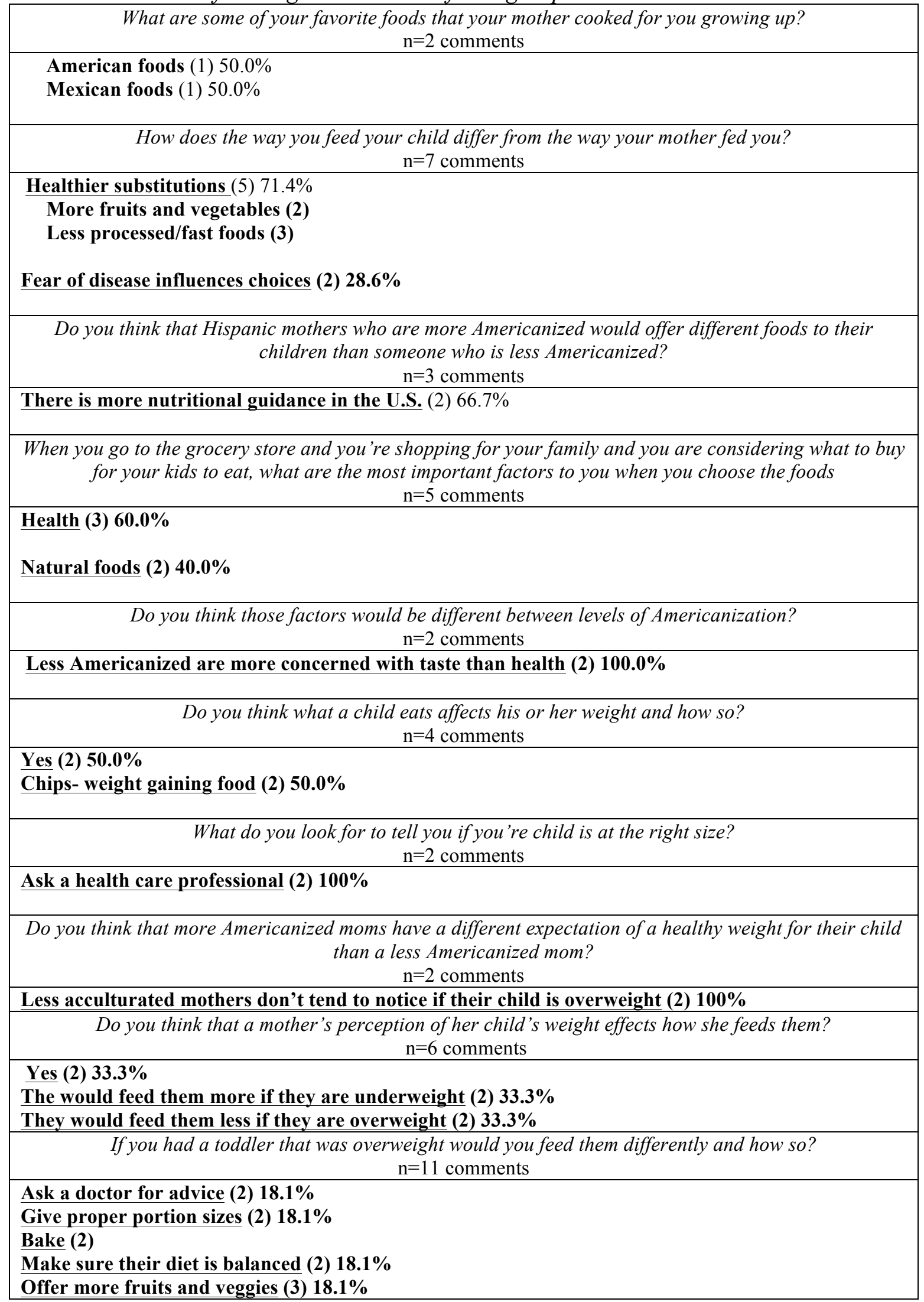


What would you do with an underweight toddler?

$n=0$ comments

No comments

Underlined themes have a percentage of total comments mentioned about the question above them.

Numbers in parenthesis are how often that specific comment was mentioned.

Bold and underlined statements are main themes, bold but not underlined statements are subthemes

\subsubsection{High acculturated interviews: themes.}

There were many themes found in the interviews of the high acculturated mothers

$(n=3)$. These mothers claimed they had made a lot of healthy changes in what they feed

their children compared to how their mothers fed them, including purchasing organic

foods, eating more fruits and vegetables, cooking with less grease and staying away from

fast foods. When these mothers were asked how they could tell if their child is at a

healthy weight, about $33 \%$ of the comments were about the child's physical ability. If the

child can be active throughout the day and is able to do developmentally appropriate

tasks (rolling over, pushing themselves up) the mothers felt that their child is at a good

weight. Consulting a health care provider was never mentioned.

When talking about less Americanized mothers, the idea of tradition arose

multiple times. A major theme was that less Americanized mother desire to stick with

tradition which leads them to choose and cook only the foods that are familiar to them.

They also believed that less Americanized mothers worry less about health and weight

than more Americanized mothers. The mothers also believed that less Americanized

mothers view a chubby baby as cute and are more likely to feed a baby anytime the child

is crying. A list of the main themes can be found in Table 4-23. 
Table 4-23 Themes from 3 interviews with high-acculturated mothers.

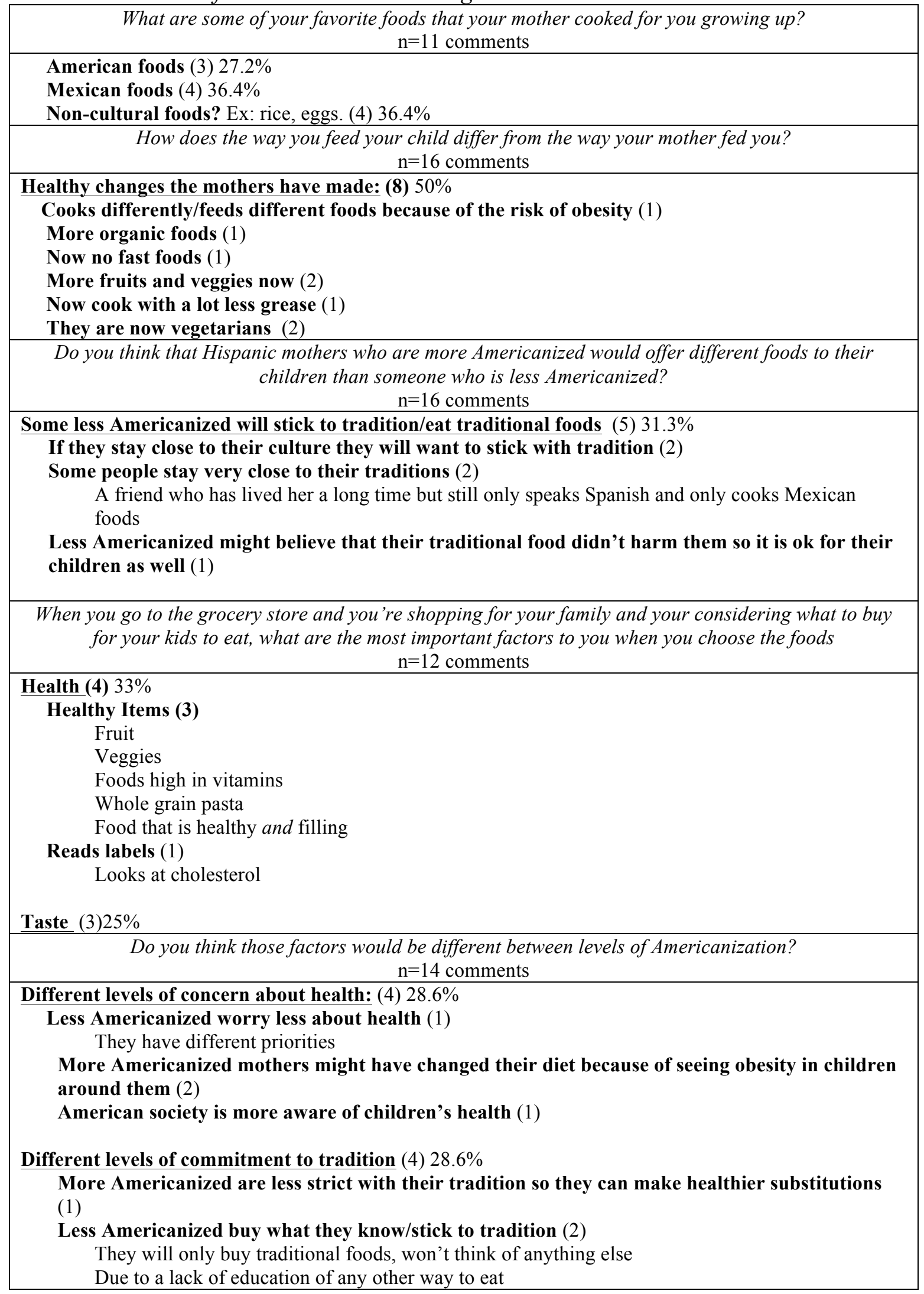




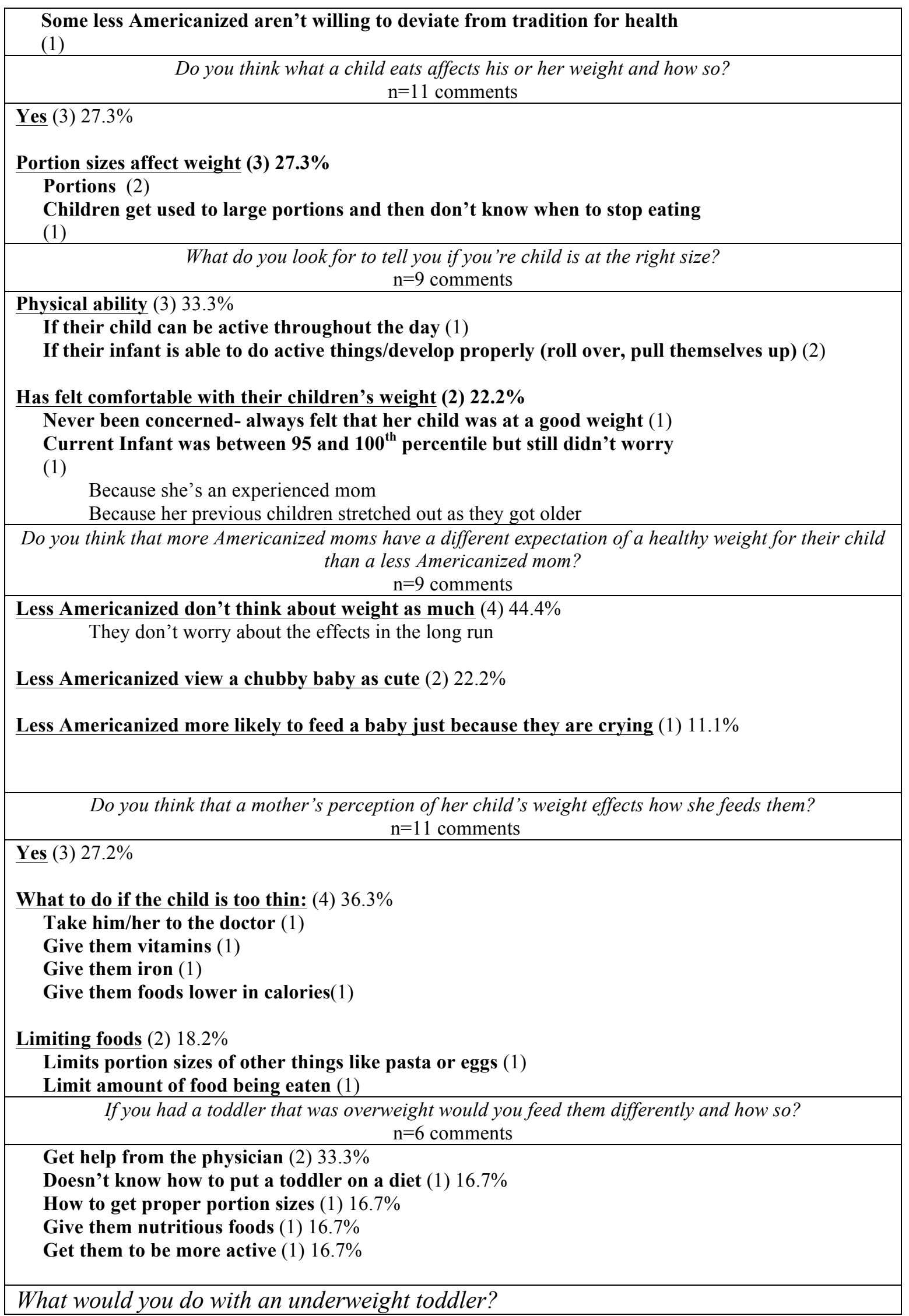




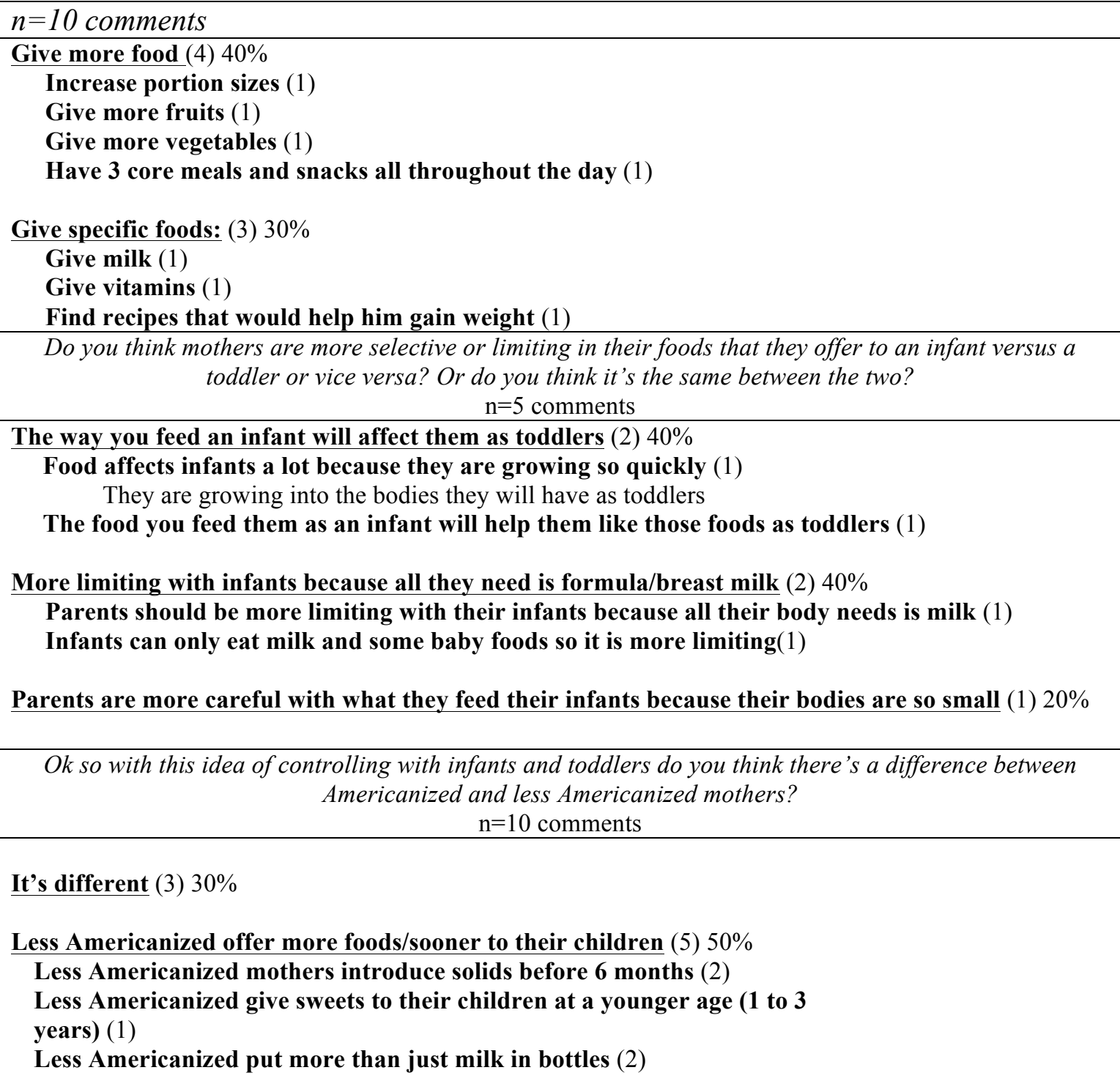

Underlined themes have a percentage of total comments mentioned about the question above them. Numbers in parenthesis are how often that specific comment was mentioned.

Bold and underlined statements are main themes, bold but not underlined statements are subthemes 


\section{CHAPTER 5}

\section{DISCUSSION}

In this study we found that acculturation had no significant effects on children's food consumption, maternal perceptions of body size or parenting stress levels. However, we found that with an increase in child's age there was an increase in the consumption of both "healthy" and "less healthy" food items. We also found that an increase in maternal BMI was correlated with a decrease in child's consumption of "healthy" food items. Acculturation did not have a significant effect on maternal accuracy in her perceptions of her child but it did have a slight direction of effect in her perceptions of herself- as mother's acculturation increased she may have been more accurate in her understanding of her own weight. Acculturation did not have a significant affect on parenting stress. Parenting stress scores were, in general, very low and none of the variables measured (acculturation, mother's age, child's age, mother's BMI or mother's education level) had a significant effect on stress levels. Key informants believed, despite the data, that acculturation level would affect what mothers fed their children and that Hispanics, regardless of acculturation level, would view a "chubby" baby as a healthy baby.

Through focus groups, we found that many mothers tried to cook the same way their mothers had cooked for them but ran into a few barriers including a change of flavors in the U.S. versus Mexico and children's preferences for American foods. The low acculturated mothers mentioned having a lot more food in the U.S. than they did in Mexico and that they feed their children more than they were fed. Both groups mentioned that more acculturated mothers would feed their children more fast foods and convenience foods than less acculturated mothers. Both groups stated that health was a 
major factor that influenced what foods they purchased for their children but the low acculturated groups also mentioned cravings affected what foods they purchased. A majority of mothers mentioned getting information on the height and weight of their children from their doctors but many of the high acculturated mothers mentioned instances in which they didn't believe their doctor's assessment of the appropriateness of their child's weight. Bi acculturated mothers mentioned that less Americanized moms are less concerned about the weight of their children and the low acculturated mothers claimed they felt uneducated in how much their children should weigh. Both groups agreed that more Americanized mothers are more likely to limit foods they give to their children or are more careful in what they feed their children and that less Americanized will give more foods to their infants and introduce solids earlier.

\subsection{Objective 1: Child Food Intake}

In the present study, only $16 \%$ of children consumed less than either 1 fruit, vegetable or legume per day. However, once we separated the vegetables, fruits and legumes about $36 \%$ of children consumed less than 1 vegetable a day, and $34 \%$ consumed less than 1 fruit a day. A study conducted on 3,273 U.S. children from birth to 3 years old found that about $30 \%$ of their participants ate less than 1 distinct vegetable a day and an additional $26 \%$ of children consumed less than 1 distinct fruit a day (Fox, Condon, Briefel, Reidy, \& Deming, 2010). In the present study, the children were reported to be consuming, on average, a little less fruit and vegetable servings a day than the general U.S. population, however, the U.S. data was collected from children between the ages of 2 and 3 while our sample was in children between the ages of 6 months and 3 years of age. In the present study, not including younger children would probably 
increase the average consumption of all foods in general as our data has shown, the older children consume more foods.

In general, consumption of only 3 healthy items per day do not meet the requirements of the dietary guidelines which recommend consuming 1 cup of fruits and vegetables a day the equivalent to about 5 servings from a variety of fruits and vegetables (USDA, 2011a, 2011b). Important reasons to eat more fruits and vegetables include their high levels of many vitamins and minerals and low levels of calories, making them very nutrient dense (U.S. Department of Agriculture \& U.S. Department of Health and Human Services, 2010). In addition an increased consumption of fruits and vegetables in children is often associated with a decrease consumption of high fat/sugar foods. Therefore, if the children are not receiving calories from nutrient dense foods, they are likely to be taking in more of the high calorie, high fat and high sugar foods which can increase the rates of obesity (Epstein et al., 2001).

In the analysis of the frequency of consumption of less healthy and healthy food consumption, only child's age had a significant positive relationship for both food groups and maternal BMI had a significant negative effect for only the healthy food groups. As a child grew older, they consumed more of the less healthy food items as well as more of the healthy food items. This is most likely because the younger children included in this analysis were around 6 months of age and had just begun to eat solids so the older children were eating an increased variety and more total foods overall. It has been shown that early exposure to fruits and vegetables can lead to an increase in acceptance of those fruits and vegetables (Forestell \& Mennella, 2007; Skinner, Carruth, Bounds, Ziegler, \& Reidy, 2002). Therefore, the early stages of feeding which were studied in the present 
research are crucial to child's later dietary habits. Ideally, by 9 months of age an infant should be consuming at least 3-4 servings of fruits and vegetables per day.

Two previous studies have shown that an increase in maternal BMI is correlated with less healthy food choices in children (Birch \& Fisher, 1995; Contento, Basch, \& Zybert, 2003). As the mother's BMI increased, the amount of healthy food items they gave to their children decreased. In a study of 187 low-income Latina women with children five to seven years old, the authors found that mothers with higher BMI's had children with lower healthy index scores, indicating that health was rated as a less important criteria in food choices (Contento et al., 2003). This supports our findings that mothers with higher BMI had children who consumed less of the healthy food items. It may be that health is less important for these mothers and they are offering less healthy foods to their children. Although mothers food intake was not assessed in the present study, it is probable that mothers with higher BMIs are consuming less healthy foods and those are the foods that would be available in the home, therefore their children would also consume these food items. An increase in BMI has also been shown to be correlated with a decrease in access to chain grocery stores so mothers of high BMI may have less access to fresh fruits and vegetables (Wang, Kim, Gonzalez, MacLeod, \& Winkleby, 2007).

This study did not show a relationship between food items consumed by children and their mother's level of acculturation. Other research has shown that as Mexican American adults acculturate into the U.S. they consume more "junk food" including more soda (Duffey et al., 2008; Himmelgreen, Brentall, Perez-Escamilla, Peng, \& Bemudez, 2005) and more fast food (G. Ayala, Mueller, Lopez-Madurga, Campbell, \& Elder, 2005; 
Duffey et al., 2008), which were some of the foods described in the "less healthy" food category in the present study. The previous studies looked at how acculturation affected Mexican American adults whereas the present study wanted to see if the acculturation of the adults affected what their children were fed. This may be one reason our study did not have similar findings. Studies done on acculturation and the diets of children are limited and we failed to find any conducted on children 3 years and younger. Studies done with children five years and older however, found that more acculturated children consume more sodium (suggesting more processed foods) (Liu et al., 2012; Pérez-Escamilla, 2009), less fruit (Liu et al., 2012; Morello et al., 2012), less vegetables and beans and more saturated fat (Liu et al., 2012).

There are a few possibilities as to why we did not see the effect acculturation on healthy or less healthy foods when other studies did. For one, we had a small sample size and may not have been able to detect the effect of acculturation on food consumption. The earlier studies had much larger sample sizes from 147 up to 3,375 participants (G. Ayala et al., 2005; Duffey et al., 2008; Himmelgreen et al., 2005). Another issue is in how acculturation is measured. The present study used country of origin and language preference to determine acculturation level and our participants were considered to be of either high or low acculturation. Some of the previous studies also used language preference and place of birth (Duffey et al., 2008; Himmelgreen et al., 2005), however, one used the ARSMA scale (G. Ayala et al., 2005). In order to not overanalyze the data, we combined the less healthy foods group to form a composite score. The previously mentioned research looked at specific foods like sugar and fast foods separately (G. X. 
Ayala et al., 2008; Duffey et al., 2008; Pérez-Escamilla \& Putnik, 2007) which we were not able to do.

It would make sense that if less acculturated adults, in general, eat more traditional foods, then their children should be consuming more of these foods as well (Burt \& Hertzler, 1978; Rozin, Fallon, \& Mandell, 1984). The focus groups and key informant interviews agreed that the level of acculturation would influence how mothers fed their children, however, research has shown that adult preferences for foods do not always affect the child's food preferences (Rozin, 1991). A study conducted on Latina mothers and their children showed that acculturation (measured by years of residency in the U.S.) had a much stronger relationship with mother's dietary intake than her child's dietary intake (Guendelman \& Siega-Riz, 2002).

The mothers in the focus groups did mention difficulties in continuing to cook their traditional food, even though they stated they cook the way their mothers had taught them. One common theme was that their children had begun to prefer American foods. Other focus group research has suggested that Hispanic mothers face opposition from their children when trying to feed them healthy foods (Maynard, Galuska, Blanck, \& Serdula, 2002). Some of the focus group mothers commented on making American foods even though they themselves did not like them. A key informant commented that "if the parents immigrated here from Mexico they are going to stick to more traditional foods because that is what they are comfortable with but their children who attend school and are much more intermixed with people who have been here for a long time they are going to want to be like their peers...”. It is possible that although the parents are sticking to more traditional foods they are giving their children American foods. 
If mothers are not passing on traditional diets, including more fruits, vegetables and legumes to their children, this is a potential area for intervention. It has been shown that second generation immigrants have, on average, lower Healthy Eating Index scores than first generation immigrants (Aldrich \& Variyan, 2000). There are negative changes occurring in the diet of the second-generation immigrants and these changes could begin with the mother providing her child with less healthy American foods.

\subsection{Objective 2: Maternal Perceptions of Body Size}

In general, the figures mothers chose to represent a typical Hispanic woman in their neighborhood were slightly overweight; the figures the mothers chose to represent a healthy and desirable body weight were healthy and the scores they chose to represent themselves were on the higher end of healthy, near a BMI of $25 \mathrm{~kg} / \mathrm{m}^{2}$. The two perceptions that varied significantly by acculturation were for the typical Hispanic woman and the healthy Hispanic woman; in both instances the high acculturated mothers chose larger figures than the low acculturated mothers. Only one other study was found which asked Latina mothers to state which figure they believed looked healthiest from a figure scale containing 7 figures. The mothers chose an average score very close to the middle figure (2.58) which was similar to our findings (Contento et al., 2003). Other studies have not looked at the effect of acculturation on maternal perceptions however one study looked to see if weight preferences differed by ethnicity using a 9 figural scale much like the one used in the current research. In this study the Hispanic women preferred an image with a score of 3.9 (Cachelin, Rebeck, Chung, \& Pelayo, 2002). This score was similar to the preference scores of $3.2( \pm 0.8)$ and $3.6( \pm 0.9)$ chosen by the low acculturated and high acculturated women respectively in the current research. 
No other research has looked at the effect of acculturation on how a woman views a typical body size in her neighborhood or a healthy body size. It was surprising to find that for both questions, the high acculturated mothers chose higher scores. It has been shown that acculturation is correlated with an increase in BMI (Fuentes-afflick \& Hessol, 2008; Liu et al., 2012; Van Hook et al., 2012) so possibly the more acculturated women spend more time with other more acculturated women or may live in neighborhoods with other low Socio Economic Status (SES) white women and may be surrounded by people with a higher BMI. This would explain why they believe other Hispanics in their neighborhood are larger and being around people with a higher BMI has influenced their view on a healthy body size.

In general, mothers tended to underestimate their own body sizes and mothers with higher BMIs were more likely to underestimate their own body weight. On average mothers underestimated their body size by 5.5 BMI units. A previous study of 187 lowincome Latina women with children from 5 to 7 years old had similar findings: the obese women described themselves as larger than the normal weight and underweight women but still tended to underestimated their own body weight (Contento et al., 2003). None of the obese women in that study believed themselves to be at risk of heart disease (Contento et al., 2003) which also suggests an inadequate understanding of their size and health. That study did not state any possible reasons for these results.

Interestingly there was a significant relationship between child's age and mother's perception of herself. The older the child, the more accurate the mother was in her perception of herself. It may be that mothers with younger children (infants) or who are breastfeeding are less concerned with their weight because of their recent pregnancy 
weight gain. Although this has not been documented previously in the literature, in focus groups of Latina women, some women said they had tried to maintain a healthy weight during pregnancy but gave up because of the desire for a larger, healthier baby (Thornton et al., 2006). Perhaps the mothers are less concerned with their weight soon after pregnancy because the weight gain was believed to be positive for their child's health. In the unadjusted analysis, we found that mothers chose figures all within the healthy range for each of the questions about children/infant body sizes: typical infant/child, healthy infant/child, preferred infant/child and which represents your infant/child. These results were very similar to the results of another study conducted on 105 African American women and their infants. Table 5-1 below shows their findings side-by-side the findings of this research (Boyington \& Johnson, 2004).

Table 5-1 Maternal perception of infant scores aged 0-12 months old.

\begin{tabular}{l|l|l|l} 
& $\begin{array}{l}\text { Mean Low } \\
\text { Acculturated } \\
\text { Score (SD) }\end{array}$ & $\begin{array}{l}\text { Mean High } \\
\text { Acculturated } \\
\text { Score (SD) }\end{array}$ & $\begin{array}{l}\text { Mean scores } \\
\text { of African } \\
\text { American } \\
\text { Study }\end{array}$ \\
\hline $\begin{array}{l}\text { Typical infant } \\
\text { Healthy infant }\end{array}$ & $3.1(1.1)$ & $3.7(0.7)$ & $3.2(1.18)$ \\
Preferred infant & $2.5(0.9)$ & $3.0(0.7)$ & $2.9(0.78)$ \\
$\begin{array}{l}\text { Represents your } \\
\text { infant }\end{array}$ & $2.7(0.9)$ & $3.1(0.9)$ & $2.9(0.83)$ \\
\hline
\end{tabular}

Adapted from Boyington and Johnson. (Boyington \& Johnson, 2004).

Similar to maternal perceptions of women's body size, when asked about a typical infant/child in their neighborhood and which infant/child they believed to be healthy, the high acculturated women chose larger figures for both children and infants compared to the low acculturated women. This could again be due to the increase in BMI that is associated with an increase in acculturation (Fuentes-afflick \& Hessol, 2008; Liu et al., 2012; Van Hook et al., 2012). The children in the high acculturated neighborhoods may 
be larger than the children in the low acculturated neighborhoods. The healthy infant score given by the high acculturated was a 3.0 which is correlated with the $50^{\text {th }}$ percentile whereas the mothers of low acculturation chose a figure which is correlated with the $10^{\text {th }}$ to the $25^{\text {th }}$ percentile, on the leaner side of normal. In the focus groups, the low acculturated mothers mentioned feeling uneducated in the area of healthy body sizes. Perhaps these women are unsure of what a healthy body size is. It is important to take into account, as well, that this data is unadjusted and has not looked at any other possible confounding variables, which could change the results.

In general, $80 \%$ of parents tended to underestimate their infant/child's weight by an average of a whole percentile range. The tendency of mothers to underestimate their children's weight has been seen in other studies conducted on Latina American women (Contento et al., 2003; Killion, Hughes, Wendt, Pease, \& Nicklas, 2006; Manios et al., 2010; Rosas et al., 2010) and is believed to be due in part to an unwillingness to classify their own children as overweight (Manios et al., 2010; Myers \& Vargas, 2000) or in difficulty choosing a picture that looked like their own child (Rosas et al., 2010). As previously reviewed, there is a Mexican cultural ideal that suggests that a chubby baby is a healthy baby (Chaparro et al., 2011), which was backed up by our key informant interview and focus group findings. These mothers may believe their children to be healthy when they are, in fact, overweight; this may interfere with how they perceive their child's body size.

As mother's BMI increased, she was more likely to underestimate not only her own weight but the weight of her child as well which is consistent with previous research (Boyington \& Johnson, 2004; Manios et al., 2010). The key informant interviewees 
suggested that perhaps overweight parents do have a genetic and environmental component affecting their weight and therefore may be surrounded by overweight family. This then influences their view to see an overweight child as normal.

\subsubsection{Acculturation and perceptions of body size}

High acculturation had only a marginally significant positive effect on the mother's ability to perceive her own weight accurately and no significant effect on her accuracy in perceiving her child's body size. There has not been a lot of research on acculturation and accuracy of women's perceptions of their own body size, however, one study did have similar findings to ours. The authors compared Latina mothers who had immigrated to the U.S. before the age of 16 , to non-Hispanic white and Latina mothers who moved to the U.S. later in life, they found the more recent immigrants underestimated their body weight while the others were accurate (Lopez et al., 1995). There has been some research on differences in ethnicity and accuracy in perceptions of body size. In one analysis conducted on European American and Hispanic women, there was no difference between women's perception by ethnicity- there was an equal amount of women from both groups who perceived themselves as normal weight, overweight and obese (Ceballos \& Czyzewska, 2010). In another study of over 5,000 men and women, $64.8 \%$ of the white women who were overweight correctly categorized themselves as overweight compared to only $45.6 \%$ of the overweight Hispanic women (Paeratakul, White, Williamson, Ryan, \& Bray, 2002). This supports the idea that the more western culture is more aware of being overweight than the Hispanic culture, which indicates that as women acculturate they will become more aware of their weight status. 
In the focus groups, we did not explore the women's view of themselves but we did explore their view of their children. Many of the less Americanized mothers felt less educated in this issue and unsure of what their children should weigh. Several other comments were made regarding less Americanized women not thinking about weight or being less aware about weight and having other priorities like trying to get to know the new culture. This may be why we saw that more acculturated mothers were more aware of their weight than the less acculturated mothers. One of the key informant interviewees believed that if a mother were more acculturated she would be more educated and aware about weight issues because this is something that is valued in the U.S. culture. This is another possible explanation for the more acculturated women being more accurate in their estimation of their own weight.

\subsection{Objective 3: Parenting Stress}

In general, the participant's chose answers that indicated low parenting stress.

Only one participant chose moderate stress levels on both the child related stress score and the parent related stress score. It is possible that our Parenting Feelings Index was not a valid measurement of parenting stress. The Parenting Feelings Index was adapted from the PSI-SF and has not been tested for validity. Other studies that measured stress and hopelessness in Mexican Americans and other low-income populations however found low scores on their indices consistent with findings in the present study. A study conducted on white, African American and Hispanic WIC participants found that about $75 \%$ of all women's responses to a stress scale were "never" or "almost never" stressed (Hurley et al., 2008). Another study looked at hopelessness (which is usually correlated with stress and depression) in 136 Mexican-American mothers. The authors found that 
only one of their participants had high levels of hopelessness and $<10 \%$ reported medium levels of stress (Marsiglia et al., 2011).

During the key informant interviews, many of the interviewees believed that the less acculturated parents would have more parenting stress. They believed that learning the new culture would add a lot to their stress levels and that they would be less knowledgeable of where to get help for their children. Our parenting stress index asked mainly questions about how the child's personality affected them and how they felt about their parenting skills. It did not measure issues someone would face when they are acculturating. This could be a reason we did not see high stress scores among our participants however it is very possible this population has good stress coping mechanisms.

Farley and colleagues found that Mexican immigrants were more likely to use "positive reframing", denial and religion as coping strategies than either non-Hispanic whites and Mexican Americans, however Mexican Americans were more likely to use these strategies than non-Hispanic whites (Farley, Galves, Dickinson, \& Perez, 2005). Positive reframing which takes a negative situation and tries to see positive aspects of it (University of Miami, 2007) was associated with better physical functioning in this study but not with mental health scores. The Mexican Americans, however, had better mental health reports than the non-Hispanic white participants. This study also found that there was no difference in perceived stress between the groups even though the Mexican immigrants were undocumented workers with many other stressors including: introduction to a new culture, fear deportation and working in "low-end" jobs (Farley et al., 2005). 
In a study of college students, the third or fourth generation students had the highest rates of acculturation stress compared with the students who were early immigrants (Mena, Padilla, \& Maldonado, 1987). The two groups also used different coping strategies. The later generation students used individualistic approaches whereas early immigrants used both social network and individualistic approaches (Mena et al., 1987). Thus the Mexican culture may be better at coping with stress than the Western culture.

Additionally, perhaps both low acculturated and high acculturated mothers are equally stressed but for different reasons. One key informant interviewee stated: "I've seen parents when they first arrive and they're stressed for many other reasons like fears and not knowing where they're going to go. Whether they're going to survive in the new country. And then later down the road I see people who have been here for about a year or so and they're still stressed about getting to their appointments because they don't have a car or they don't know how to use the bus system. So their stress levels are sort of the same but for different reasons." Our sample size was very small and a lot of the mothers left multiple questions unanswered in the stress test so it would be beneficial to conduct more research on this topic, and validate the instrument.

\subsection{Aim 3: Focus Groups}

Theme: mother's had much less food growing up in Mexico and now feed their children much more than what they were fed.

Mothers in the low acculturated focus groups mentioned many times that they have a lot more food in the U.S. than they had growing up in Mexico. They mentioned feeding their children more food including having dessert after dinner. It may be that 
these mothers want to give their children what they didn't have growing up. One of our key informant interviewees mentioned how mothers saw fast food and junk food as a treat they can give their children that they themselves didn't have in Mexico. Some research has been conducted on how food insecurity affects feeding patterns. One study conducted in low income Mexican-American women showed that current food insecurity was associated with less monitoring of sweets for their children (Crawford et al., 2007). Another study on Latino and non-Latino white women showed that past food insecurity was associated with less monitoring of both sweets and snacks for their children (Kuyper, Smith, \& Kaiser, 2009). These findings however did not hold once they controlled for mother's education and household income.

\section{Theme: Health is the most important consideration when choosing food for their children.}

When the mothers were asked what they look for when purchasing foods, they mentioned health $36 \%$ of the time in the low acculturated groups and over $50 \%$ of the time in the bi-acculturated focus groups. Health was by far the most common topic mentioned within this question for both groups, but it was especially common in the high-acculturated groups. There were also many comments throughout the focus groups about how more Americanized mothers are more concerned with health and weight than the less Americanized mothers. This thought is supported by the large portion of health related comments made in the bi-acculturated group.

Although health seems to be of primary importance to these mothers, according to the data from Aim 1 on dietary intake, children are not consuming enough fruits and vegetables. We found that children were only consuming fruits, vegetables and legumes 
half as often as is recommended, on average consuming a little over 3 total servings daily, compared with the recommended 5-6 servings a day. Aldrich and Variyan found that Spanish speaking Hispanic adults highly valued a healthy diet especially when compared to non-Hispanic whites and English speaking Hispanics. In spite of this value for health, their Healthy Eating Index Scores was below the ideal range (Aldrich \& Variyan, 2000). This supports our findings that although these women hold health as an important value, the diets of their children may not reflect this value.

\section{Theme: Genetics matter most in influencing body size (more so than diet).}

While many of the low acculturated mothers believed that food affects children's weight, there was also a large number who believed that genetics had a greater effect on weight than diet. They mentioned experiences with genetics such as having a child that can eat anything they want and not gain weight or two children who eat the same but one is overweight and the other isn't. The belief that genetics affect weight the most was found in another focus group of Latina mothers with preschool children (Crawford et al., 2007). This perception, in combination with the cultural ideal that a "chubby baby is a healthy baby" and the tendency for parents to underestimate their child's weight, suggests it may be beneficial to direct education towards how to raise a healthy child and not to focus on maintaining a healthy weight. Other research has come to similar conclusions and has recommended connecting nutrition and health with increased happiness or family happiness (Crawford et al., 2004). This connection may be a more useful motivational tool for families to change their diet. 


\section{Theme: Mothers look to health care professionals to inform them about the weight of their child.}

When we asked the mothers how they decide if their child is at a healthy weight, a majority of the comments stated they receive information about weight from the doctor or other health care professionals. This suggests that doctors and health care professionals play a big role in Mexican-American mother's awareness of their child's body size. One study showed that only $18 \%$ of mothers with overweight children recall being informed about the weight of their child from the doctor (Eckstein et al., 2006). If doctors and health care professionals do have an important influence on mother's knowledge of appropriate weight, which our study suggests, it is important for health care professionals to be more consistent in reporting and discussing children's weight to the parents.

Some mothers from the bi-acculturated group, however, reported that they did not believe their doctor when they were told their child was overweight. These mothers believed their child was underweight despite what the doctor said. Respect for authority is an important Hispanic value (Clutter \& Nieto, 2006) and a high respect for doctors as authority figures in Hispanic culture has also been recorded (Austin, Ahmad, \& Stewart, 1994). The bi-acculturated group's willingness to question the doctor's authority may be a sign of their acculturation and movement away from traditional Hispanic values.

The mother's disbelief of the doctor's weight assessment for their children supports our earlier findings that mothers tend to underestimate their child's body size and supports the idea mentioned earlier that focusing on weight when trying to educate mother's on healthy food choices may not be beneficial for this population. However, many of low acculturated mothers felt that they did not know how to assess their child's 
weight on their own and would like more education in this area. Educating mothers on a healthy weight for their child-- separate from education on nutritious food-- may be helpful for this group in order to prevent early childhood obesity.

\section{Theme: Less Americanized mothers give more foods to infants than more Americanized moms.}

Another common theme found in the focus groups, and supported by the interviews with high acculturated mothers, is that less Americanized mothers will introduce solids earlier or give more food to their infants. The data analysis under aim 1 did not explore the age children first received solids but the FITS study in 2006 (Ziegler, Hanson, Ponza, Novak, \& Hendricks, 2006) had a sample size of 3,022 infants and toddlers and found that the Hispanic infants were more likely to receive meat, fruit and fruit juices before 6 months of age. A focus group of Latina women admitted having fed their infants solids before 6 months even though the doctor had not recommended it. They had done so based on the advice given them by their mothers (Chaidez, Townsend, \& Kaiser, 2011).

\subsection{Strengths and Limitations}

\section{Strengths:}

This study has several strengths. First, it combines quantitative data with qualitative data, allowing for both a quantifiable look at foods offered to and consumed by infants and children, maternal perceptions of body size and parenting stress as well as a rich data set from the focus groups. The focus group data helps explain why we are seeing certain feeding habits and maternal perceptions in the low income Mexican- 
American population including why children are not consuming different foods based on their mother's acculturation level and why a higher level of maternal BMI may increase her likelihood in underestimating her child's body size as well as her own. Second, this study looked at several variables that have not been heavily explored previously including the effect of parent's acculturation level on child's food intake and the effect of acculturation on parenting stress. Third, focus groups were divided by the participant's acculturation level, which is also unique to this area of investigation and allowed for the comparison of the two groups qualitatively.

\section{Limitations:}

This study has several limitations. Both the Aim 1 and Aim 3 portions of this study had small sample sizes ( $\mathrm{n}=68$ and $\mathrm{n}=32$ respectively). The small sample size lessened the power making it difficult to pick up on small but potentially "significant" relationships. This may be why we did not see a relationship between acculturation and many of the other variables.

The use of acculturation measurements is another limitation of this study. For aim 1 we used country of origin and primary language to determine if our mothers were in the "high acculturated" or "low acculturated" categories. Although this method has been reported in the literature (Chaparro et al., 2011; Liu et al., 2012), this does not take into account many other factors that contribute to acculturation. Later when we used the BAS method to determine level of acculturation for the focus group mothers very few of the mothers could be considered highly acculturated according to this measurement. Most of the mothers were either bi acculturated or low acculturated. So our earlier categorization of acculturation, in the cross-sectional study using 2 variables high and low, may not 
have been ideal and probably would have given us different results had we used the BAS. For the cross-sectional study, out of the 107 recruits, 23 were born in the U.S. and using the BAS method 8 of those women were considered to be of high acculturation, whereas using our earlier criteria, all 23 would have been considered of high acculturation. It is likely that the level of acculturation was overestimated in the cross-sectional study.

Another limitation of our study was that we used only WIC and Head Start participants. These families in general are of low income and would not be representative of the Mexican-American population as a whole. These mothers were all receiving similar nutrition education from WIC and Head Start staff, which may have biased answers to our focus group questions about healthy eating and healthy body size. It is possible that the participants answered our questions with responses they believed we wanted to hear.

The instrument we developed to measure parenting stress was also limited. It is possible it did not measure areas of parenting stress that are affecting this population the most which led to very low stress scores.

Lastly, our focus groups had a very high attrition rate (67\%), which was higher than the expected. The drop-outs were marginally significantly different than participants in country of origin and marital status. Recruits were more likely to participate if they were from Mexico and if they were married. Therefore, we may have missed out on the inputs of U.S. born women of Latina descent and single mothers so these findings are not generalizable to those populations. 


\subsection{Recommendations for future education:}

Based on our findings, there are several possible areas for education in the WIC and Head Start Latina population.

1. Stages of growth: since mothers did claim they felt uneducated about their child's weight and some mothers claimed they disagreed with doctors who said their child was overweight, it might be beneficial to teach these mothers about the proper stages of normal growth for infants and young children and possibly some of the consequences of early excess weight gain.

2. Giving your child healthy treats: another area for education is demonstrating how to give your child treats that are truly treats, rather than food items that are potentially providing excess energy and may be detrimental for food habit development. Real "treats" could include trips to the park, playing a game together, letting their child choose a fruit or vegetable from the produce aisle or reading a book together. It would be important to emphasize that just because something was not available to a mother when she was young does not mean it is an appropriate treat for their child. It would be better to indulge the children with love/attention rather than food.

3. Passing on tradition to your child. Mothers could be shown how to incorporate their child's preferences into healthy yet traditional foods. Helping the mothers to feel empowered by allowing them to feel in control of their child's growth and development may encourage the mothers to cook according to their value of tradition and health instead of cooking what will please their child. It could also 
be beneficial to emphasize the practice of not making separate food for their children but that everyone at the dinner table should be eating the same thing.

4. When to introduce solids: Mothers should be educated on the consequences of introducing solids early (increased risk of obesity and food allergies) and making sure they know the signs showing when their infant is ready to advance to baby foods and solids. It would also be beneficial to involve grandmothers or other caregivers in this education because they may have a significant impact on the mother's parenting and food choices.

\subsection{Recommendations for Future Research}

In order to determine if the key messages we have suggested would be beneficial to WIC and Head Start families, they should be developed, implemented and then checked for effectiveness. Since there was no previous evidence in the literature on maternal dietary acculturation having an influence on their child's dietary acculturation, it would also be interesting to compare the role of maternal acculturation on her diet and on that of her child by assessing food frequencies of both the mother and the child. This would allow us to see if children's dietary acculturation is affected by maternal dietary acculturation or if our hypothesis that children and mothers are eating separate food is indeed correct. It would also be beneficial to develop a more sensitive parenting stress instrument, which addresses culturally appropriate areas of stress for Latina mothers. Lastly, replicating Aim 1 of our research but with a larger sample size might allow us to see if there truly is a difference between acculturation and our tested variables: food frequency of children 3 years and under, maternal perceptions of body size and parenting stress levels. 


\subsection{Conclusions}

When considering the influence of acculturation on food frequency of children less than 3 years, child's diet, maternal perceptions of body size and parenting stress levels, this study showed few correlations between maternal acculturation and the diet of their children, their perceptions of body size and parenting stress levels. Results showed the children were not consuming adequate amounts of fruits and vegetables and that an increase in maternal BMI was associated with a decreased consumption of healthy foods by their children. Mothers often underestimated their own body weight as well as that of their child especially if they had a high BMI. It is possible that these children are being introduced to the American lifestyle early on and many of the benefits of their parent's dietary traditions are not being passed on to the next generation, mainly a diet higher in fiber, fruit and vegetables. More research needs to be done in this area.

During the focus groups we found that the low acculturated mothers had much less food growing up in Mexico than they currently have in the U.S. and the mothers may be giving more food to their children as a treat with the intention of giving to their children what they were unable to have growing up. In addition many of the low acculturated mothers believed that genetics affects the weight of their children much more so than diet. Mothers in both the low and the bi acculturated groups believed that low acculturated mothers worry less about weight and health than more acculturated mothers. Lastly, mothers from both acculturation groups mentioned that less acculturated mothers would be feeding their infants more solids and are more likely to introduce solids early than more acculturated mothers. 
Areas of possible intervention include education on the proper stages of growth, promoting the continuation of healthy traditional foods, developing effective education on health promoting treats for parents to give their children and educating parents on the consequences of early introduction to solids and benefits of waiting until 6 months of age to introduce solids.

Funding: This study was funded from California State WIC and Agriculture Research Initiative, Cal Poly San Luis Obispo. 


\section{REFERENCES}

Abidin, R. R. (1983). Parenting Stress Index (PSI) Administration Booklet (pp. 1-14).

About WIC. (2012). Food \& Nutrition Services. Retrieved January 02, 2012, from http://www.fns.usda.gov/wic/aboutwic/

Acevedo, M. C. (2000). The role of acculturation in explaining ethnic differences in the prenatal health-risk behaviors, mental health, and parenting beliefs of Mexican American and European American at-risk women. Child Abuse \& Neglect, 24(1), 111-127. doi:http://dx.doi.org/10.1016/S0145-2134(99)00121-0

Aldrich, L., \& Variyan, J. (2000). Acculturation errodes the Diet Quality of U.S. Hispanics. Food Review, 23(1), 51-55.

Allen, L. (1992). The Mexican Food System: Traditional and Modern. Ecology of Food and Nutrition, 27, 219-234.

Arcia, G. J., Crouch, L. A., \& Kulka, R. A. (1990). Impact of the WIC program on Food Expenditures. American Journal of Agricultural Economics, 72(1), 218-226.

Ariza, A. J., Chen, E. H., Binns, H. J., \& Christoffel, K. K. (2004). Risk factors for overweight in five- to six-year-old Hispanic-American children: a pilot study. Journal of urban health : bulletin of the New York Academy of Medicine, 81(1), 150-61. doi:10.1093/jurban/jth091

Austin, L. T., Ahmad, F., \& Stewart, D. E. (1994). Breast and Cervical Cancer Screening in Hispanic Women : Cancer Supplement, 74(1), 323-328.

Ayala, G., Mueller, K., Lopez-Madurga, E., Campbell, N., \& Elder, J. (2005). Restaurant and food shopping selections among Latino Women in Southern California. Journal of the American Dietetic Association, 105, 38-45.

Ayala, G. X., Baquero, B., \& Klinger, S. (2008). A systematic review of the relationship between acculturation and diet among Latinos in the United States: implications for future research. Journal of the American Dietetic Association, 108(8), 1330-44. doi:10.1016/j.jada.2008.05.009

Birch, L., \& Fisher, J. O. (1995). Fat preferences and fat consumption of 3- to 5- year-old children are related to parental adiposity. Journal of the American Dietetic Association, 95(7), 759.

Boyington, J. A., \& Johnson, A. a. (2004). Maternal perception of body size as a determinant of infant adiposity in an African-American community. Journal of the National Medical Association, 96(3), 351-62. Retrieved from 
http://www.pubmedcentral.nih.gov/articlerender.fcgi?artid=2594865\&tool=pmcentr ez\&rendertype $=$ abstract

Brewis, A. (2003). Biocultural aspects of obesity in young Mexican schoolchildren. American Journal of Human Biology, 15(3), 446-60. doi:10.1002/ajhb.10161

Briefel, R., Ziegler, P., Novak, T., \& Ponza, M. (2006). Feeding Infants and Toddlers Study: characteristics and usual nutrient intake of Hispanic and non-Hispanic infants and toddlers. Journal of the American Dietetic Association, 106(1 Suppl 1), S84-95. doi:10.1016/j.jada.2005.09.040

Bureau, U. S. C. (2010). No Title. American Community Survey Reports, U.S. Department of Commerce, Economics and Statistics Administration The foreignborn population in the United States. Retrieved from http://www.census.gov/prod/2012pubs/acs-19.pdf

Burt, J. V., \& Hertzler, A. A. (1978). Parental influence on the child's food preference. Journal of Nutrition Education, 10(4), 127-128. doi:10.1016/S00223182(78)80141-1

Cachelin, F. M., Rebeck, R. M., Chung, G. H., \& Pelayo, E. (2002). Does ethnicity influence body-size preference? A comparison of body image and body size. Obesity Research, 10(3), 158-66. doi:10.1038/oby.2002.25

Cali, A., \& Caprio, S. (2008). Obesity in Children and Adolescents. Journal of Clinical Endocrinology and Metabolism, 11, 531-536.

California WIC. (2012). Program At a Glance (pp. 1-4).

California WIC. (2013). WIC Income Guideline Table. WIC Appendix 980-1060. Retrieved from http://www.slocounty.ca.gov/Assets/PH/wic+pdf+file/WICIncomeGuidelines_4-1-2013+to+6-30-14.pdf

Campos, B., Schetter, C. D., Walsh, J. a., \& Schenker, M. (2007). Sharpening the Focus on Acculturative Change: ARSMA-II, Stress, Pregnancy Anxiety, and Infant Birthweight in Recently Immigrated Latinas. Hispanic Journal of Behavioral Sciences, 29(2), 209-224. doi:10.1177/0739986307300841

CDC. (2011a). Healthy Weight- it's not a diet, it's a lifestyle! About BMI for Adults.

CDC. (2011b). Health Weight- it's not a diet, it's a lifestyle! About BMI for Chidlren and Teens. Retrieved August 07, 2013, from http://www.cdc.gov/healthyweight/assessing/bmi/childrens_bmi/about_childrens_b mi.html\#What is BMI percentile 
CDC. (2013). Childhood Obesity. Adolescent and School Health. Retrieved August 07, 2013, from http://www.cdc.gov/healthyyouth/obesity/facts.htm

Ceballos, N., \& Czyzewska, M. (2010). Body Image in Hispanic/Latino Vs. European American Adolescents: Implications for Treatment and Prevention of Obesity in Underserved Populations. Journal of health care for the poor and underserved, 21, 823-836.

Chaidez, V., Townsend, M., \& Kaiser, L. L. (2011). Toddler-feeding practices among Mexican American mothers. A qualitative study. Appetite, 56(3), 629-32. doi:10.1016/j.appet.2011.02.015

Chaparro, M. P., Langellier, B. a, Kim, L. P., \& Whaley, S. E. (2011). Predictors of accurate maternal perception of their preschool child's weight status among Hispanic WIC participants. Obesity (Silver Spring, Md.), 19(10), 2026-30. doi:10.1038/oby.2011.105

Chen, J. (2010). Household Income, Maternal Acculturation, Maternal Education Level and Health Behaviors of Chinese-American Children and Mothers. Journal of Immigrant and Minority Health / Center for Minority Public Health, 11(3), 198204.

Chen, L., Juon, H.-S., \& Lee, S. (2012). Acculturation and BMI among Chinese, Korean and Vietnamese adults. Journal of community health, 37(3), 539-46. doi:10.1007/s10900-011-9476-1

Clutter, A. W., \& Nieto, R. D. (2006). " Understanding the Hispanic Culture ." The Ohio State University. Retrieved from http://ohioline.osu.edu/hyg-fact/5000/5237.html

Collins, J., \& Shay, D. (1994). Prevalence of Low Birth Weight among Hispanic Infants with United States-born and Foreign-born Mothers: The Effect of Urban Poverty. American Journal of Epidemiology, 139(2), 184-192.

Collins, M. E. (1991). Body figure perceptions and preferences among preadolescent children. Internation Journal of Eating Disorders, 10, 199-208.

Contento, I., Basch, C., \& Zybert, P. (2003). Body Image, Weight, and Food Choices of Latina Women and Their Young Children. Journal of Nutrition Education and Behavior, 35(5), 236-248.

Coronado, G. D., Thompson, B., Mclerran, D., Schwartz, S. M., \& Koepsell, T. D. (2005). A Short Acculturation Scale for Mexican-American Populations. Ethnicity \& Disease, 15, 53-62. 
Cossrow, B., \& Falkner, B. (2004). Race/ethnic issues in obesity and obesity-related comorbidities. The Journal of Clinical Endocrinology and Metabolism, 89(6), 25902594.

Crawford, P. B., Gosliner, W., Anderson, C., Strode, P., Becerra-Jones, Y., Samuels, S., ... Ritchie, L. D. (2004). Counseling Latina mothers of preschool children about weight issues: suggestions for a new framework. Journal of the American Dietetic Association, 104(3), 387-94. doi:10.1016/j.jada.2003.12.018

Crawford, P., Lamp, C., Nicholson, Y., Krathwohl, S., Hudes, M., \& Townsend, M. (2007). Food insecurity may be linked to childhood obesity in low-income MexicanAmerican families. California Agriculture, 61(3), 106-111.

Curiel, A. A., Levy, T. S., \& Vlliasana, A. C. (1995). Encuesta urbana de alimentacion y nutricion en la zona metropolitana de la cuidad de Mexico.

Dabrowska, a, \& Pisula, E. (2010). Parenting stress and coping styles in mothers and fathers of pre-school children with autism and Down syndrome. Journal of intellectual disability research : JIDR, 54(3), 266-80. doi:10.1111/j.13652788.2010.01258.x

De La O, A., Jordan, K. C., Ortiz, K., Moyer-Mileur, L. J., Stoddard, G., Friedrichs, M., ... Mihalopoulos, N. L. (2001). Do parents accurately perceive their child's weight status? Journal of Pediatric Health Care: Official Publication of National Association of Pediatric Nurse Associates \& Practitioners, 23(4), 216-21. doi:10.1016/j.pedhc.2007.12.014

Duffey, K. J., Gordon-larsen, P., Ayala, G. X., \& Popkin, B. M. (2008). Birthplace Is Associated with More Adverse Dietary Profiles for US-Born Than for Foreign-Born Latino Adults. The Journal of Nutrition Epidemiology, 138, 2428-2435. doi:10.3945/jn.108.097105.However

Eckstein, K. C., Mikhail, L. M., Ariza, A. J., Thomson, J. S., Millard, S. C., \& Binns, H. J. (2006). Parents' perceptions of their child's weight and health. Pediatrics, 117(3), 681-90. doi:10.1542/peds.2005-0910

Encuesta Nacional de Salud y Nutricion de Mexico. (2012). Mexican National Health and Nutrition Survey. National Public Health Institute.

Ennis, S. R., Rios-Vargas, M., \& Albert, N. G. (2011). The Hispanic Population : 2010 (pp. 1-16). Retrieved from http://www.census.gov/prod/cen2010/briefs/c2010br04.pdf

Epstein, L. H., Gordy, C. C., Raynor, H. a, Beddome, M., Kilanowski, C. K., \& Paluch, R. (2001). Increasing fruit and vegetable intake and decreasing fat and sugar intake 
in families at risk for childhood obesity. Obesity Research, 9(3), 171-8. doi:10.1038/oby.2001.18

Eschbach, K., Ostir, G. V, Patel, K. V, Markides, K. S., \& Goodwin, J. S. (2004). Neighborhood context and mortality among older Mexican Americans: is there a barrio advantage? American Journal of Public Health, 94(10), 1807-12. Retrieved from http://www.pubmedcentral.nih.gov/articlerender.fcgi?artid=1448538\&tool=pmcentr ez\&rendertype $=$ abstract

Evans, A., Chow, S., Jennings, R., Dave, J., Scoblick, K., Sterba, K. R., \& Loyo, J. (2011). Traditional foods and practices of Spanish-speaking Latina mothers influence the home food environment: implications for future interventions. Journal of the American Dietetic Association, 111(7), 1031-8. doi:10.1016/j.jada.2011.04.007

Farley, T., Galves, A., Dickinson, L. M., \& Perez, M. D. J. D. (2005). Stress, coping, and health: a comparison of Mexican immigrants, Mexican-Americans, and nonHispanic whites. Journal of Immigrant Health, 7(3), 213-20. doi:10.1007/s10903005-3678-5

Fein, S. B., Labiner-Wolfe, J., Shealy, K. R., Li, R., Chen, J., \& Grummer-Strawn, L. M. (2008). Infant Feeding Practices Study II: study methods. Pediatrics, 122 Suppl , S28-35. doi:10.1542/peds.2008-1315c

Fitzgibbon, M. L., Blackman, L. R., \& Avellone, M. E. (2000). The relationship between body image discrepancy and body mass index across ethnic groups. Obesity research, 8(8), 582-9. doi:10.1038/oby.2000.75

Flegal, K., Carroll, C., Ogden, C., \& Curtin, L. (2010). Prevalence and trends in obesity amoung U.S. adults, 1999-2008. The Journal of the American Medical Association, 303(3), 235-241.

Forestell, C. a, \& Mennella, J. a. (2007). Early determinants of fruit and vegetable acceptance. Pediatrics, 120(6), 1247-1254. doi:10.1542/peds.2007-0858

Fox, M. K., Condon, E., Briefel, R. R., Reidy, K. C., \& Deming, D. M. (2010). Food consumption patterns of young preschoolers: are they starting off on the right path? Journal of the American Dietetic Association, 110(12 Suppl), S52-9. doi:10.1016/j.jada.2010.09.002

Fuentes-afflick, E., \& Hessol, N. A. (2008). Acculturation and Body Mass among Latina Women. Journal of Women's Health, 17(1), 67-73. doi:10.1089/jwh.2007.0389 
Garcia, C., \& Lindgren, S. (2009). "Life grows between the rocks": Latino adolescents' and parents' perspectives on mental health stressors. Research in Nursing \& Health, 32(2), 148-62. doi:10.1002/nur.20317

Grabe, S., \& Hyde, J. S. (2006). Ethnicity and body dissatisfaction among women in the United States: a meta-analysis. Psychological Bulletin, 132(4), 622-40.

doi:10.1037/0033-2909.132.4.622

Grieco, E., Acosta, Y., de la Cruz, P., Gambino, C., Gryn, T., Larsen, L., ... Walters, N. (2012). The Foreign-Born Population in the United States : 2010 (pp. 1-22).

Guendelman, S., Fernald, L. C. H., Neufeld, L. M., \& Fuentes-Afflick, E. (2010). Maternal perceptions of early childhood ideal body weight differ among Mexicanorigin mothers residing in Mexico compared to California. Journal of the American Dietetic Association, 110(2), 222-9. doi:10.1016/j.jada.2009.10.033

Guendelman, S., Fernandez, A., Thornton, D., \& Brindis, C. (2011). Birthplace, language use, and body size among Mexican American women and men: findings from the National Health and Nutrition Examination Survey (NHANES) 2001-2006. Journal of Health Care for the Poor and Underserved, 22(2), 590-605. doi:10.1353/hpu.2011.0045

Guendelman, S., \& Siega-Riz, A. M. (2002). Infant feeding practices and maternal dietary intake among Latino immigrants in California. Journal of Immigrant Health, 4(3), 137-46. doi:10.1023/A:1015698817387

Guinn, B., Semper, T., Jorgensen, L., \& Skaggs, S. (1997). Body Image Perception in Female Mexican-American Adolescents. Journal of School Health, 67(3), 112-115.

Gupta, V. B. (2007). Comparison of Parenting Stress in Different Developmental Disabilites. Journal of Developmental and Physical Disabilites, 19(4), 417-425.

Haskett, M. E., Ahern, L. S., Ward, C. S., \& Allaire, J. C. (2006). Factor structure and validity of the parenting stress index-short form. Journal of Clinical Child and Adolescent Psychology: the Official Journal for the Society of Clinical Child and Adolescent Psychology, American Psychological Association, Division 53, 35(2), 302-12. doi:10.1207/s15374424jccp3502_14

Hassan, D., \& Hekmat, S. (2012). Dietary Acculturation of Arab Immigrants in the Greater Toranto Area. Canadian Journal of Dietetic Practice and Research, 73(3), $143-146$.

Hedley, A. a, Ogden, C. L., Johnson, C. L., Carroll, M. D., Curtin, L. R., \& Flegal, K. M. (2004). Prevalence of overweight and obesity among US children, adolescents, and adults, 1999-2002. JAMA : the Journal of the American Medical Association, 291(23), 2847-50. doi:10.1001/jama.291.23.2847 
Hernández-Valero, M. a, Wilkinson, A. V, Forman, M. R., Etzel, C. J., Cao, Y., Bárcenas, C. H., ... Bondy, M. L. (2007). Maternal BMI and country of birth as indicators of childhood obesity in children of Mexican origin. Obesity (Silver Spring, Md.), 15(10), 2512-9. doi:10.1038/oby.2007.298

Himmelgreen, D. A., Brentall, A., Perez-Escamilla, R., Peng, Y., \& Bemudez, A. (2005). Birthplace, length of time in the U.S., and language are associated with diet among inner-city Puerto Rican Women. Ecology of Food and Nutrition, 44, 105-122.

Huang, J. S., Becerra, K., Oda, T., Walker, E., Xu, R., Donohue, M., ... Breslow, A. (2007). Parental ability to discriminate the weight status of children: results of a survey. Pediatrics, 120(1), e112-9. doi:10.1542/peds.2006-2143

Hurley, K. M., Black, M. M., Papas, M. a, Caulfield, L. E., \& Caufield, L. E. (2008). Maternal symptoms of stress, depression, and anxiety are related to nonresponsive feeding styles in a statewide sample of WIC participants. The Journal of Nutrition, 138(4), 799-805. Retrieved from http://www.pubmedcentral.nih.gov/articlerender.fcgi?artid=3137941\&tool=pmcentr ez\&rendertype $=$ abstract

Irigoyen, M., Glassman, M., Chen, S., \& Findley, S. (2008). Early onset of overweight and obesity among low-income 1 - to 5- year olds in new york city. Journal of Urban Health, 86(4), 545-554.

Jones, J. (2010). South Africa in the 19th Century. West Chester University. Retrieved June 05, 2013, from http://courses.wcupa.edu/jones/his312/lectures/southafr.htm

Killion, L., Hughes, S. O., Wendt, J. C., Pease, D., \& Nicklas, T. a. (2006). Minority mothers' perceptions of children's body size. International Journal of Pediatric Obesity, 1(2), 96-102. doi:10.1080/17477160600684286

Kuyper, E., Smith, D., \& Kaiser, L. (2009). Does Food Insecurity Influence Child Feeding Practices? Journal of Hunger \& Environmental Nutrion, 4(2), 147-157.

Landale, N, Oropesa, R. S., \& Gorman, B. K. (2000). Migration and Infant Death: Assimilation or Selective Migration among Puerto Ricans? American Sociological Review, 65(6), 888-909.

Landale, Nancy, Oropesa, R. S., Llanes, D., \& Gorman, B. (1999). Does Americanization Have Adverse Effects on Health?: Stress, Health Habits, and Infant Health Outcomes among Puerto Ricans*. Social Forces, 78(2), 613-641.

Lind, C., Merchandani, G., Castrucci, B., Chavez, N., Handler, A., \& Hoelscher, D. (2012). The Effects of Acculturation on Healthy Lifestyle Characterisits Among Hispanic Fourth-Grade Children in Texas Public Schools , 2004-2005. Journal of School Health, 82(4), 166-174. 
Liu, J., Chu, Y. H., Frongillo, E. A., \& Probst, J. C. (2012). Generation and Acculturation Status Are Associated with Dietary Intake and Body Weight in Mexican American Adolescents. The Journal of Nutrition, 298-305. doi:10.3945/jn.111.145516.higher

Lopez, E., Blix, G. G., \& Blix, A. G. (1995). Body image of Latinas compared to body image of non-Latina white women. Health Values: The Journal of Health Behavior, Education \& Promotion, 19(6), 3-10. Retrieved from http://search.ebscohost.com/login.aspx?direct=true\&db=rzh\&AN=1996028233\&site $=$ ehost-live

Lopez-Gonzalez, A. L., Aravena, V. C., Hummer, R. A., \& Lopez-gonzalez, L. (2005). Immigrant Acculturation, Gender and Health Behavior: A Research Note. Social Forces, 84(1), 581-593.

Manios, Y., Moschonis, G., Grammatikaki, E., Anastasiadou, A., \& Liarigkovinos, T. (2010). Determinants of childhood obesity and association with maternal perceptions of their children's weight status: the "GENESIS" study. Journal of the American Dietetic Association, 110(10), 1527-31. doi:10.1016/j.jada.2010.07.004

Marin, G., \& Gamba, R. J. (1996). A New Measurement of Acculturation for Hispanics: The Bidimensional Acculturation Scale for Hispanics (BAS). Hispanic Journal of Behavioral Sciences, 18(3), 197-316.

Marsiglia, F. F., Kulis, S., Perez, H. G., \& Bermudez-Parsai, M. (2011). Hopelessness, Family Stress, and Depression among Mexican-Heritage Mothers in the Southwest. Health \& Social Work, 36(1), 8-18.

Maynard, M., Galuska, D., Blanck, H., \& Serdula, M. (2002). Maternal Perceptions of Weight Status of Children. The American Academy of Pediatrics, 111, 1226-1231.

McDermott, A., Hey, D., Teaford, S., \& Minarik, W. (2009). Barriers to Physical Activity and Healthy Eating As Perceived by Parents in Lompoc: Focus Group Results (pp. 1-53). Retrieved from http://digitalcommons.calpoly.edu/cgi/viewcontent.cgi?article=1027\&context=kine fac\&seiredir $=1 \&$ referer $=$ http $\% 3 \mathrm{~A} \% 2 \mathrm{~F} \% 2 \mathrm{Fscholar}$.google.com $\% 2 \mathrm{Fscholar} \% 3 \mathrm{Fq} \% 3 \mathrm{DMcDe}$ rmott\%2BHey\%2BTeaford\%26btnG\%3D\%26hl\%3Den\%26as_sdt\%3D0\%252C5\#s earch $=\% 22 \mathrm{McDermott}$ Hey Teaford $\% 22$

McKelvey, L., Whiteside-Mansell, L., Faldowski, R.A., Shears, J., Ayoub, C., \& Hart, A. D. (2009). Validity of the short for of the parenting stress index for fathers of toddlers. Journal of Child and Family Studies, 18(1), 102-111.

Mena, F., Padilla, A., \& Maldonado, M. (1987). Acculturative stress and specific coping strategies among immigrant and later generation college students. Hispanic Journal of Behavioral Sciences, 9, 207-225. 
Montez, J. K., \& Eschbach, K. (2008). Country of Birth and Language are uniquely Associated with Intakes of Fat, Fiber, and Fruits and Vegetables among MexicanAmerican Women in the United States. Journal of the American Dietetic Association, 103(3), 473-480.

Morello, M. I., Madanat, H., Crespo, N. C., Lemus, H., \& Elder, J. (2012). Associations among parent acculturation, child BMI, and child fruit and vegetable consumption in a Hispanic sample. Journal of Immigrant and Minority Health / Center for Minority Public Health, 14(6), 1023-9. doi:10.1007/s10903-012-9592-8

Myers, S., \& Vargas, Z. (2000). Parental perceptions of the pre-school obese child. Pediatric Nursing, 26(30).

National WIC Association. (2013). How WIC Impacts the People of California (pp. 1-2). Retrieved from http://www.paramountcommunication.com/nwica/California.pdf

NIH. (2011). What is Metabolic Syndrome? National Institues of Health. Retrieved from http://www.nhlbi.nih.gov/health/health-topics/topics/ms/

Olaiz, G., Rivera, J., Teresa, S., Rosalba, R., Villalpando, S., Hernandez, M., \& Sepulveda, J. (2006). Encuesta Nacional de Salud y Nutricion (pp. 1-131).

Olvera, N., Suminski, R., \& Power, T. G. (2005). Intergenerational perceptions of body image in hispanics: role of BMI, gender, and acculturation. Obesity Research, 13(11), 1970-9. doi:10.1038/oby.2005.242

Ostberg, M., Hagekull, B., \& Hagelin, E. (2007). Stability and Prediction of Parenting Stress. Infant and Child Development, 16, 207-223. doi:10.1002/icd

Paeratakul, S., White, M. a, Williamson, D. a, Ryan, D. H., \& Bray, G. a. (2002). Sex, race/ethnicity, socioeconomic status, and BMI in relation to self-perception of overweight. Obesity Research, 10(5), 345-50. doi:10.1038/oby.2002.48

Papathakis, P., Nazmi, A., Phelan, S., \& Engle, P. (2013). When obesity begins: anthropometric and demographic characteristics of California WIC child participants.

Park, S.-Y., Murphy, S. P., Sharma, S., \& Kolonel, L. N. (2005). Dietary intakes and health-related behaviours of Korean American women born in the USA and Korea: The Multiethnic Cohort Study. Public Health Nutrition, 8(07), 904-911. doi:10.1079/PHN2005740

Pérez-Escamilla, R. (2009). Dietary quality among Latinos: is acculturation making us sick? Journal of the American Dietetic Association, 109(6), 988-91. doi:10.1016/j.jada.2009.03.014 
Pérez-Escamilla, R., \& Putnik, P. (2007). The role of acculturation in nutrition, lifestyle, and incidence of type 2 diabetes among Latinos. The Journal of Nutrition, 137(4), 860-70. Retrieved from http://www.ncbi.nlm.nih.gov/pubmed/17374645

Petti, Y. M., \& Cowell, J. M. (2011). An assessment of measures of body image, body attitude, acculturation, and weight status among Mexican American women. Journal of the American Academy of Nurse Practitioners, 23(2), 84-91. doi:10.1111/j.17457599.2010.00581.x

Pierce, B. L., Austin, M. a, Crane, P. K., Retzlaff, B. M., Fish, B., Hutter, C. M., ... Fujimoto, W. Y. (2007). Measuring dietary acculturation in Japanese Americans with the use of confirmatory factor analysis of food-frequency data. The American Journal of Clinical Nutrition, 86(2), 496-503. Retrieved from http://www.ncbi.nlm.nih.gov/pubmed/17684224

Romero-Gwynn, E., \& Gwynn, D. (1993). Dietary Patterns and Acculturation Among Latinos of Mexican Descent. Nutrition Today, 28(4).

Rosas, L. G., Harley, K. G., Guendelman, S., Fernald, L. C. H., Mejia, F., \& Eskenazi, B. (2010). Maternal perception of child weight among Mexicans in California and Mexico. Maternal and Child Health Journal, 14(6), 886-94. doi:10.1007/s10995009-0534-6

Rose, D., Habicht, J., \& Devaney, B. (1998). Household Participation in the Food Stamp and WIC Programs Increases the Nutrient Intakes of Preschool Children. The Journal of nutrition, 128, 548-555.

Rozin, P. (1991). Family resenblance in food and other domains: The family paradox and the role of parental congruence. Appetite, 16(2), 93-102.

Rozin, P., Fallon, A., \& Mandell, R. (1984). Family resemblance in attitudes to foods. Developmental Psychology, 10(2), 309-314. doi:10.1037//0012-1649.20.2.309

Rush, D. (1986). The National WIC Evaluation: An Evaluation of the Special Supplemental Food Program for Women, Infants and Children. The American Journal of Clinical Nutrition, 48(2), 439-483.

Satia, J. A. (2010). Dietary acculturation and the nutrition transition: an overview. Applied Physiology Nutrition and Metabolism, 35, 219-223.

Satia-About, A. J., Patterson, R., Neuhouser, M., \& Elder, J. (2002). Applications to Nutrition Research and Dietetics. Journal of the American Dietetic Association, 102, 1105-1118.

Scribner, R., \& Dwyer, J. H. (1989). Acculturation and low birthweight among Latinos in the Hispanic HANES. American journal of public health, 79(9), 1263-7. Retrieved 
from

http://www.pubmedcentral.nih.gov/articlerender.fcgi?artid=1349701\&tool=pmcentr ez\&rendertype $=$ abstract

Skelton, J., Cook, S., Auinger, P., Klein, J., \& Barlow, S. (2009). Prevalence and trends of severe obesity among US children and adolescents. Academy of Pediatrics, 9(5), $322-329$.

Skinner, J., Carruth, B. R., Bounds, W., Ziegler, P., \& Reidy, K. (2002). Do FoodRelated Experiences in the First 2 Years of Life Predict Dietary Variety in SchoolAged Children? Journal of Nutrition Education and Behavior, 34, 310-315.

Skreden, M., Skari, H., Malt, U. F., Pripp, A. H., Björk, M. D., Faugli, A., \& Emblem, R. (2012). Parenting stress and emotional wellbeing in mothers and fathers of preschool children. Scandinavian Journal of Public Health, 40(7), 596-604. doi:10.1177/1403494812460347

Smith, T. B., Ph, D., Oliver, M. N. I., \& Innocenti, M. S. (2001). Parenting Stress in Families. American Journal of Orthopsychiatry, 71(2), 257-261.

Solis, M. L., \& Abidin, R. R. (1991). The Spanish Version Parenting Stress Index: A Psychometric Study. Journal of Clinical Child Psychology, 20(4), 372-378.

Stunkard, A. J., Sorenson, T., \& Schulsinger, F. (1983). Use of the Danish Adoption Register for the study of obesity an thinness. The Genetics of Neurological and Psychiatric Disorders, 60, 115-120.

Taveras, E., Gillman, M., Kleinman, K., Rich-Edwards, J., \& Rifas-Shiman, S. (2010). Racial/Ethnic Differences in Early Life Risk Factors for Childhood obesity. Pediatrics, 125686-125696.

Teske, R. H. C., \& Nelson, B. H. (1974). Acculturation and Assimilation : A Clarification. American Ethnologist, 1(2), 351-367.

The California WIC Program. (2010). Feed Me! Age 1 to 3. Feed Me! Age 1 to 3. Retrieved from http://www.cdph.ca.gov/programs/wicworks/Documents/NE/WICNE-EdMaterials-FeedMeAge1 to3.pdf

The California WIC Program. (2011). WIC Authorized Food List Shopping Guide. Retrieved June 06, 2013, from http://www.cdph.ca.gov/programs/wicworks/WIC Foods/WICAuthorizedFoodListShoppingGuide-7-5-2011.pdf.pdf

The California WIC Program. (2012). California Families Grow Healthy With WIC. California Families Grow Healthy With WIC. Retrieved June 06, 2013, from http://www.cdph.ca.gov/programs/wicworks/Documents/NE/WIC-NE-EdMaterialsFamiliesGrowHealthyWithWIC.pdf 
Thornton, P. L., Kieffer, E. C., Salabarría-Peña, Y., Odoms-Young, A., Willis, S. K., Kim, H., \& Salinas, M. a. (2006). Weight, diet, and physical activity-related beliefs and practices among pregnant and postpartum Latino women: the role of social support. Maternal and Child Health Journal, 10(1), 95-104. doi:10.1007/s10995005-0025-3

Tovar, A., Must, A., Metayer, N., Gute, D., Pirie, A., Hyatt, R., \& Economos, C. (2012). Immigrating to the US: What Brazilian, Latin American and Haitian Women Have to Say About Changes to Their Lifestyle That May be Associated with Obesity. Journal of Immigrant and Minority Health, 1-8. doi:10.1007/s10903-012-9665-8

U.S. Department of Agriculture, \& U.S. Department of Health and Human Services. (2010). Dietary Guidelines for Americans 2010 (pp. 1-95).

United States Census Bureau. (2013). California. State \& County QuickFacts. Retrieved July 05, 2013, from http://quickfacts.census.gov/qfd/states/06000.html

University of Miami. (2007). COPE (complete version). University of Miami. Retrieved from http://www.psy.miami.edu/faculty/ccarver/sclCOPEF.html

USDA. (2011a). How Many Vegetables Are Needed Daily or Weekly? Choosemyplate.gov. Retrieved October 06, 2013, from choosemyplate.gov/printpages.MyPlateFoodGroups/Vegetables/foodgroups.vegetables-amount.pdf

USDA. (2011b). How Many Fruits Are Needed Daily or Weekly? Choosemyplate.gov. Retrieved October 06, 2013, from choosemyplate.gov/printpages/MyPlateFoodGroups/Fruits/food-groups.fruitsamount.pdf

USDA Food and Nutrition Services. (2010). WIC Participant and Program Characteristics (pp. 1-100).

Van Hook, J., Baker, E., Altman, C. E., \& Frisco, M. L. (2012). Canaries in a coalmine: Immigration and overweight among Mexican-origin children in the US and Mexico. Social Science \& Medicine (1982), 74(2), 125-34. doi:10.1016/j.socscimed.2011.10.007

Wang, M. C., Kim, S., Gonzalez, A. a, MacLeod, K. E., \& Winkleby, M. a. (2007). Socioeconomic and food-related physical characteristics of the neighbourhood environment are associated with body mass index. Journal of Epidemiology and Community Health, 61(6), 491-8. doi:10.1136/jech.2006.051680

WIC eligibility requirements. (2012). Food \& Nutrition Services. Retrieved January 02, 2013, from http://www.fns.usda.gov/wic/howtoapply/eligibilityrequirements.htm 
Wiking, E., Johansson, S.-E., \& Sundquist, J. (2004). Ethnicity, acculturation, and self reported health. A population based study among immigrants from Poland, Turkey, and Iran in Sweden. Journal of Epidemiology and Community Health, 58(7), 57482. doi:10.1136/jech.2003.011387

Wojcicki, J. M., Schwartz, N., Jiménez-Cruz, A., Bacardi-Gascon, M., \& Heyman, M. B. (2011). Acculturation, dietary practices and risk for childhood obesity in an ethnically heterogeneous population of Latino school children in the San Francisco bay area. Journal of Immigrant and Minority Health / Center for Minority Public Health, 14(4), 533-9. doi:10.1007/s10903-011-9553-7

Ziegler, P., Hanson, C., Ponza, M., Novak, T., \& Hendricks, K. (2006). Feeding Infants and Toddlers Study: meal and snack intakes of Hispanic and non-Hispanic infants and toddlers. Journal of the American Dietetic Association, 106(1 Suppl 1), S10723. doi:10.1016/j.jada.2005.09.037 


\title{
APPENDICES
}

\section{Appendix A: Informed Consent for Aim 1}

\author{
Cal Poly State University, San Luis Obispo \\ Departments of Food Science and Nutrition and Psychology and Child Development
}

\section{INFORMED CONSENT TO PARTICIPATE IN A RESEARCH PROJECT ENTITLED: Infant and Young Child Feeding Study: WIC}

\begin{abstract}
A research project on infant and young child feeding practices is being conducted by Peggy Papathakis and Patrice Engle, in the Departments of Food Science and Nutrition and Psychology and Child Development at Cal Poly, San Luis Obispo.
\end{abstract}

The purpose of the study In this study, we want to learn about the relationship between infant and young child feeding practices and the development of overweight and obesity. We would like to find out about food and beverages your child eats and drinks, your thoughts about what a healthy baby looks like, and when and how you feed your child. We also want to learn about what you do to help your child eat, and how it feels to be a parent.

Why is this study important?

We want each child to have the best chance for a good start in life and to be well nourished. However, there are many children in California who become overweight very early in life, and this may decrease their health in the future. We want to find out what kinds of attitudes and behaviors parents have when they are feeding their children so that we can help them give their child the best care.

We are inviting a total of 80 Latina women in the WIC program who have children from 3 months to 36 months of age to participate in this study. We want to find out what Latino mothers think about feeding their children and about their children's weight in order to help them make the best choices possible for their children's feeding.

Benefits and Risks

The benefit of this study is that from the information we obtain, we will have a better idea of how to help mothers find ways to use the best feeding practices to make sure that their children are eating the best foods for them, growing well and have a healthy weight. This is important to be able to prevent the possible risks of overweight in young children. This information may help other mothers in the future who are participating in WIC.

At the end of the interview session, to thank you for your time, you will be given a $\$ 10$ gift certificate to Walmart.

There is a minor psychological risk in that it is possible that you might feel a bit uncomfortable thinking about you and your child's weight, or about how you are feeling about your baby and yourself as you answer these questions. If so, here are ways you can receive psychological services:

call 211 (an information referral hotline)

call 8055497786 for the post-partum depression support line

call 8005494499 hotline for transitions mental health

contact your primary care physician for referral

Invitation to Participate

We would also like to invite you to participate in this study. However, if you do not want to be in this study, it will not affect your benefits from WIC in any way. 
If you agree to be in this study, we will make an appointment to spend an additional 60-90 minutes with you to ask questions when you come to your next clinic visit. We will:

1. Measure your height and weight with standard scales and measures.

2. Ask you questions about which foods your child usually eats and what foods or fluids he/she ate in the previous day.

3. Ask you to identify a drawing of a healthy infant/young child and a healthy woman

4. Ask you questions about feeding style and feeding behaviors

5. Ask you questions about how you feel about your child, about parenting and yourself, and about your child's responses to the world

All of the information will be strictly confidential, and your name and that of your child will not be linked in any way with the information we collect. We will give you a code number, not using your name on any of the questionnaires. The code key will be destroyed after the data have been analyzed to further protect your confidentiality in the future.

We would like to ask your consent for WIC to release your information to us for up to one year so that we can see how your baby/child is growing.

If you agree to be interviewed for this study, we will set up an appointment time that is linked with your next regular visit. The study recruiter will call you 2 days prior to the appointment to remind you of the extended visit appointment. The recruiter today will obtain a copy of your child's WIC nutrition and family information, weight and growth measurements to date.

By participating in this study, you may be eligible for a follow-up study which would provide you with individualized infant and young child feeding counseling. We will be asking a small number of mothers-permission to visit their homes, but that will be several months later. We will ask your permission about this at that time.

The decision to be in this study or not is entirely up to you. If you do not want to participate, you are free to say, "No". At any time if you wish to stop your participation, you are free to do so without affecting your benefits from WIC. Remember, if you do not want to be in this study, it will not affect your benefits from WIC in any way.

How will we protect your confidentiality?

The information we record will only be used for research purposes, and will only be labeled with a code number. Your confidentiality will be protected and all records will be kept completely confidential in locked computers and file cabinets. Only senior research staff will have access to all the information collected. Reports of the study will not give any names or personal details. Once the study is completed, if you are interested, a copy of the reports will be available in this clinic.

Who do I contact if I want to know more, or if I have a problem at any time? If you want more information about the study before deciding whether or not to participate, or if you participated in the study and have questions, please call: Dr. Papathakis (756-

7205), Dr. Engle (756-2914), or The study coordinator: Kohleen Ervin 5418152603

If at any time you have concerns about the manner in which the research has been conducted, you may contact Dr. Steve Davis, Chair of the Cal Poly Human Subjects Committee, at 756-2754, 


$$
\begin{aligned}
& \text { Cal Poly State University, San Luis Obispo } \\
& \text { Departments of Food Science and Nutrition and Psychology and Child Development }
\end{aligned}
$$

sdavis@calpoly.edu, or Dr. Susan Opava, Dean of Research and Graduate Programs, at 756-1508, sopava@calpoly.edu.

I, _ agree to participate in the study

(print your name)

"Infant and Young Child Feeding Study: WIC

I have received and understand the study information sheet, and I understand that this study involves research.

I have discussed the advantages and disadvantages of participating in the study.

I agree to have my body measured for height and weight.

I agree to answer questions about foods my child eats.

I agree to answer questions about feeding styles, child temperament and stress.

I agree to allow WIC to provide you with information about me and my child that they have collected already.

I agree to allow WIC to provide my contact information to you for up to one year.

I am aware that I am free to leave the study at any time without any consequence. I will still be able to attend and make full use of all the facilities at the WIC clinic as usual. I freely agree to participate in this study.

Signed:

Date:

Name:

Witness

Signed:

Date:

Name:

Please keep one copy of this form for future reference. The signed copy will be kept in our study office.

Mother's WIC ID:

Child's WIC ID: 


\section{Appendix B: Food Frequency Questionnaires}

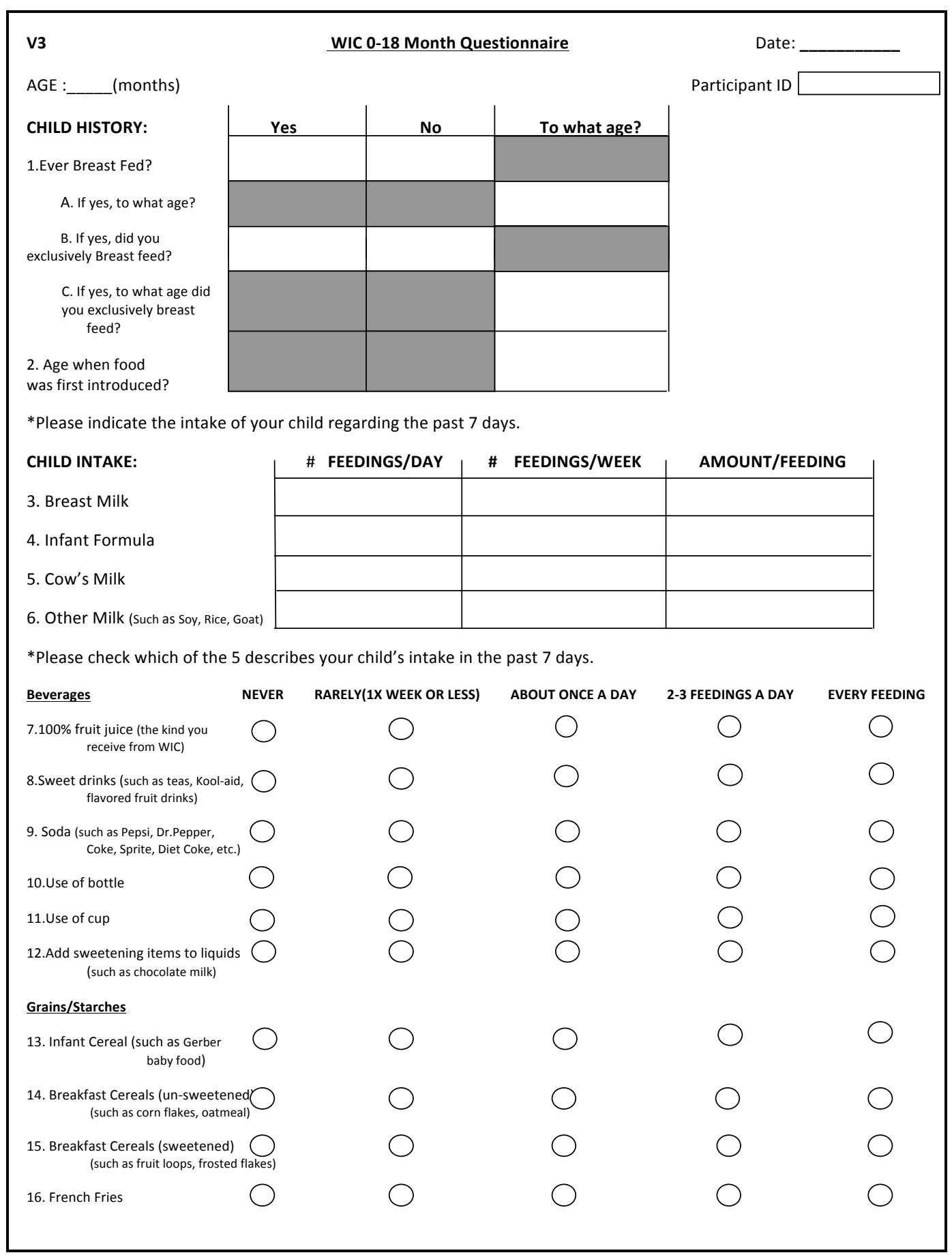




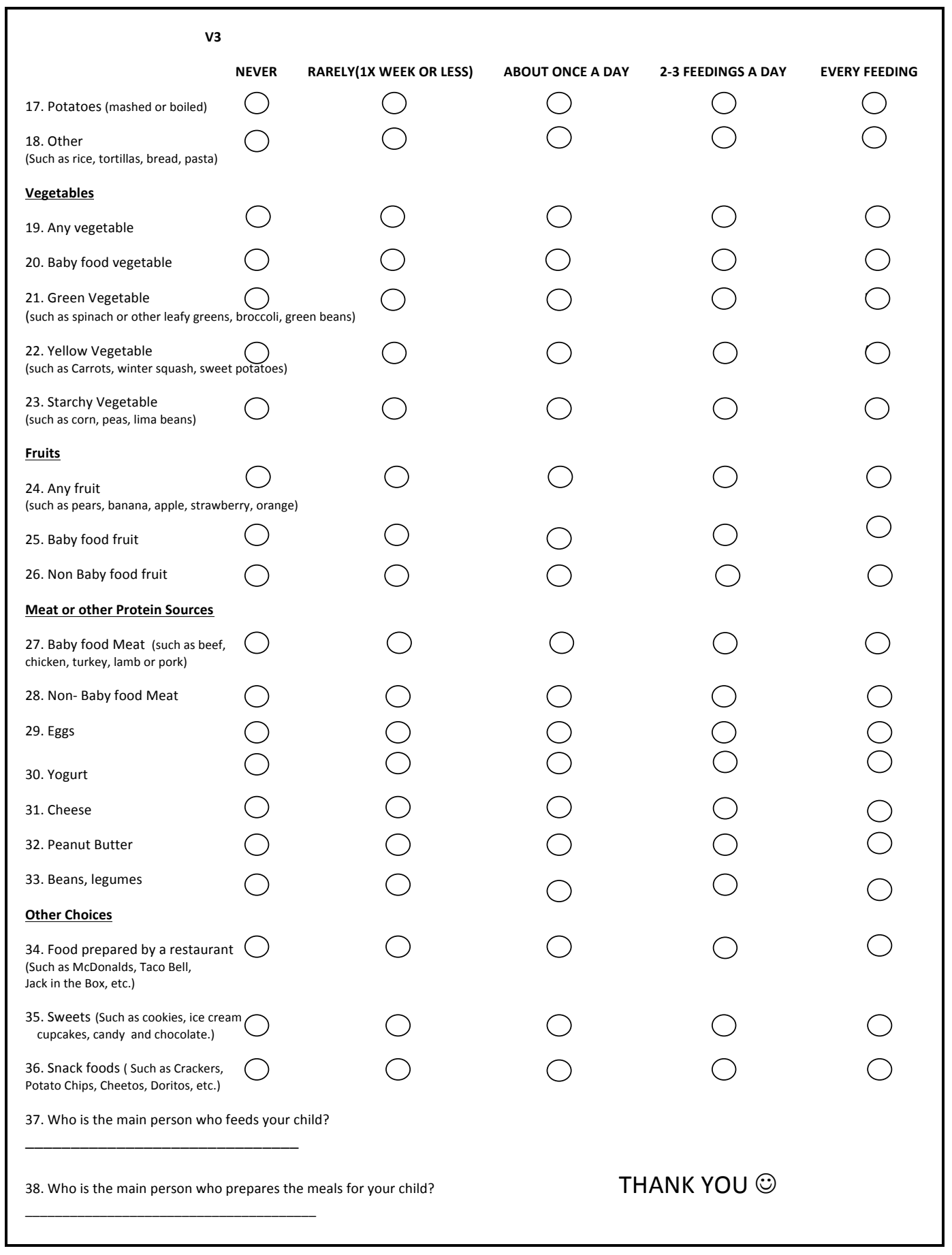




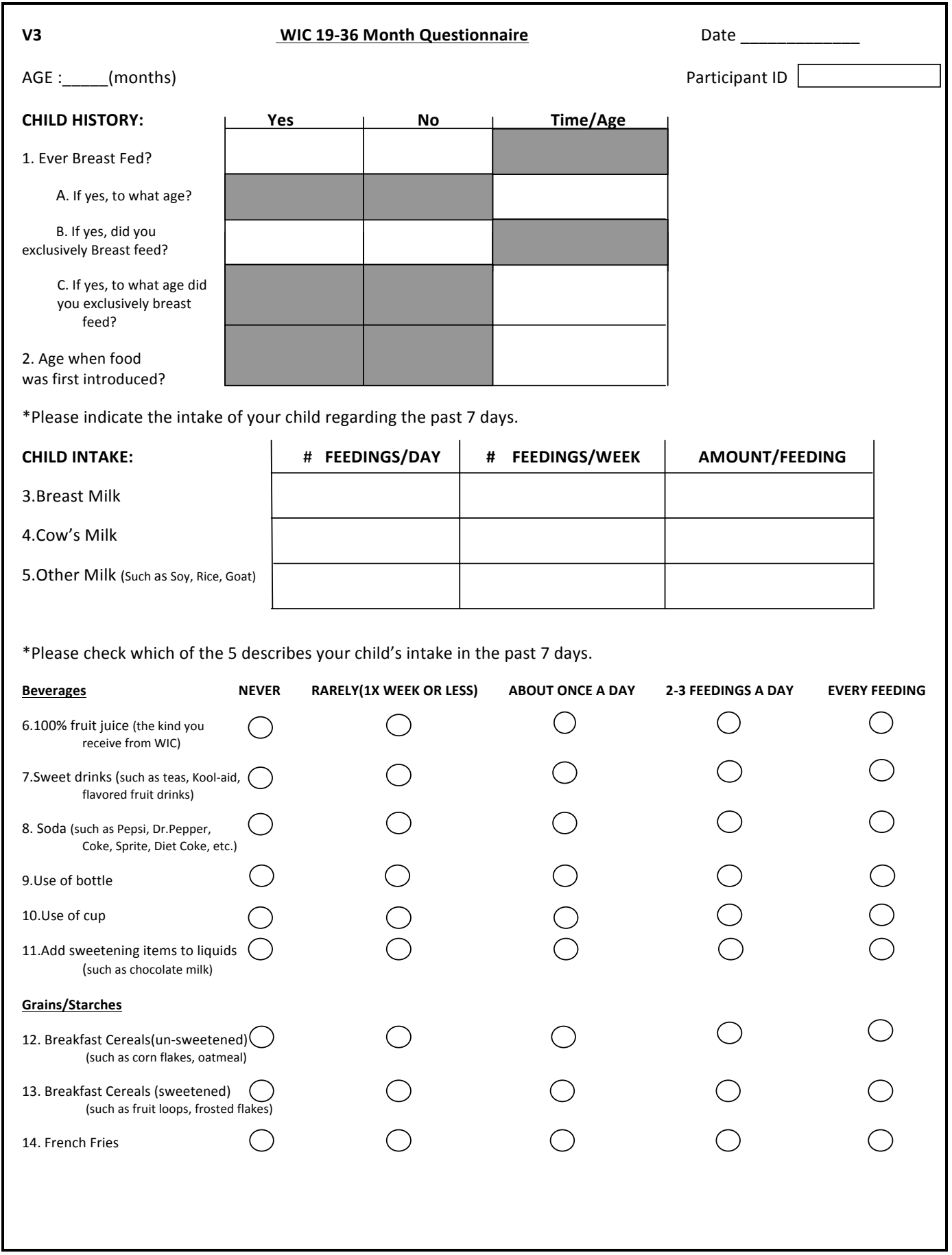




\begin{tabular}{|c|c|c|c|c|}
\hline NEVER & RARELY(1X WEEK OR LESS) & ABOUT ONCE A DAY & 2-3 FEEDINGS A DAY & EVERY FEEDING \\
\hline 15. Potatoes (mashed or boiled) & $\bigcirc$ & $\bigcirc$ & $\bigcirc$ & 0 \\
\hline $\begin{array}{l}\text { 16. Other } \\
\text { (Such as Rice, Tortilla, bread, pasta) }\end{array}$ & $\bigcirc$ & $\bigcirc$ & $\bigcirc$ & $\bigcirc$ \\
\hline \multicolumn{5}{|l|}{ Vegetables } \\
\hline 17. Any vegetable $\quad \bigcirc$ & $\bigcirc$ & $\bigcirc$ & $\bigcirc$ & $\bigcirc$ \\
\hline \multicolumn{5}{|c|}{$\begin{array}{l}\text { 18. Green Vegetable } \\
\text { (such as spinach or other leafy greens, broccoli, green beans) }\end{array}$} \\
\hline \multicolumn{5}{|l|}{$\begin{array}{l}\text { 19. Yellow Vegetable } \\
\text { (such as carrots, winter squash, sweet potatoes) }\end{array}$} \\
\hline $\begin{array}{l}\text { 20. Starchy Vegetable } \\
\text { (such as corn, peas, lima beans) }\end{array}$ & $\bigcirc$ & $\bigcirc$ & $\bigcirc$ & $\bigcirc$ \\
\hline \multirow{2}{*}{\multicolumn{5}{|c|}{$\begin{array}{l}\text { 21. Any fruit } \\
\text { (such as pears, banana, apple, strawberry, orange) } \\
\text { Meat or other Protein Sources }\end{array}$}} \\
\hline & & & & \\
\hline $\begin{array}{l}22 \text { Meat (such as beef, chicken, } \\
\text { turkey, lamb or pork) }\end{array}$ & $\bigcirc$ & $\bigcirc$ & $\bigcirc$ & $\bigcirc$ \\
\hline 23. Eggs & $\bigcirc$ & $\bigcirc$ & $\bigcirc$ & ○ \\
\hline 24. Yogurt & $\bigcirc$ & $\bigcirc$ & $\bigcirc$ & $\bigcirc$ \\
\hline 25. Cheese & $\bigcirc$ & $\bigcirc$ & $\bigcirc$ & $\bigcirc$ \\
\hline 26. Peanut Butter & $\bigcirc$ & $\bigcirc$ & $\bigcirc$ & $\bigcirc$ \\
\hline $\begin{array}{l}\text { 27. Beans, legumes } \\
\text { Other Choices }\end{array}$ & $\bigcirc$ & $\bigcirc$ & $\bigcirc$ & $\bigcirc$ \\
\hline $\begin{array}{l}\text { 28. Food prepared by a } \\
\text { restaurant (Such as McDonalds, Taco Bell, } \\
\text { Jack in the Box, etc.) }\end{array}$ & $\bigcirc$ & $\bigcirc$ & $\bigcirc$ & $\bigcirc$ \\
\hline $\begin{array}{l}\text { 29. Sweets (Such as cookies, ice cream } \\
\text { cupcakes, candy and chocolate.) }\end{array}$ & $\bigcirc$ & $\bigcirc$ & $\bigcirc$ & $\bigcirc$ \\
\hline $\begin{array}{l}\text { 30. Snack foods: ( Such as Crackers, } \\
\text { Potato Chips, , heeetos, Doritos, etc.) } \\
\text { 31. Who is the main person who feeds your }\end{array}$ & child? & $\bigcirc$ & $\bigcirc$ & $\bigcirc$ \\
\hline \multicolumn{5}{|c|}{ 32. Who is the main person who prepares the meals for your child? } \\
\hline \multicolumn{5}{|c|}{ THANK YOU :-; } \\
\hline
\end{tabular}




\title{
Appendix C: Maternal perceptions forms
}

\author{
Maternal Perception of infant ( $<12$ months old) \\ V2 \\ Participant ID \\ Infant and young child feeding study: WIC \\ Date: \\ Questions to ask the mother about the body appearance of her infant (Record her response number \\ next to each question):
}

1. Which of these figures do you think represents a typical Hispanic male or female infant in your neighborhood?

2. Which of these figures do you think represents a healthy body size for a male or female infant in your neighborhood?

3. Which of these figures do you prefer your baby to look at 6-9 months?

4. Which of these figures do you think represents the size of your baby?

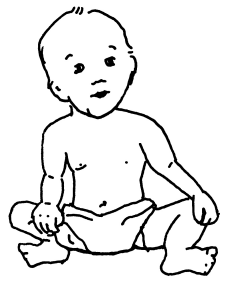

1

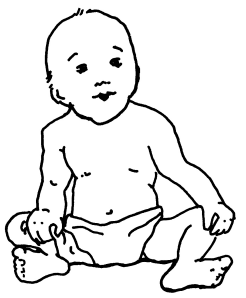

2

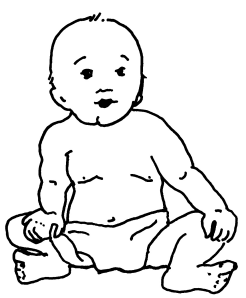

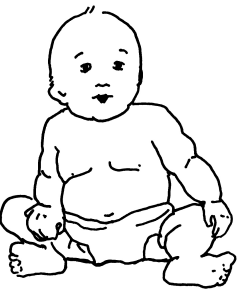

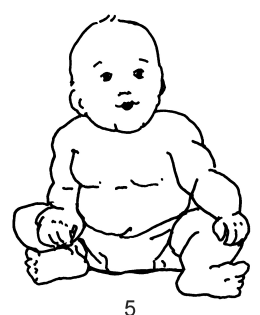


Maternal Perception of young child (1-3 years) V2

Participant ID
Infant and young child feeding study: WIC

Date:

Questions to ask the mother about the body appearance of her infant (Record her response number next to each question):

1. Which of these figures do you think represents a typical Hispanic male or female 1-3 year old in your neighborhood?

2. Which of these figures do you think represents a healthy body size for a male or female 1-3 year old in your neighborhood?

3. Which of these figures do you prefer your baby to look at 1-3 year old?

4. Which of these figures do you think represents the size of your child?

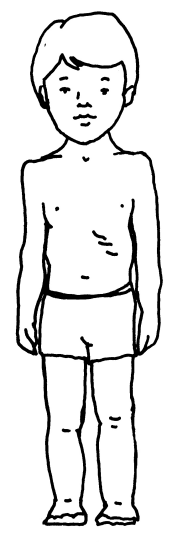

1

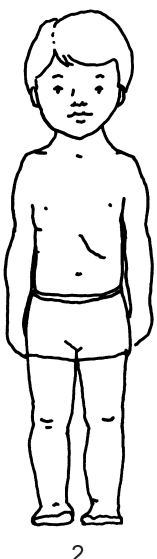

2

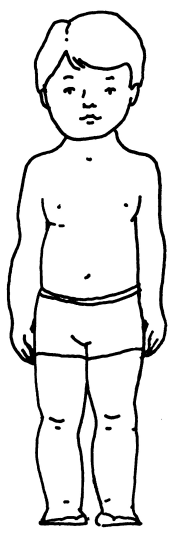

3

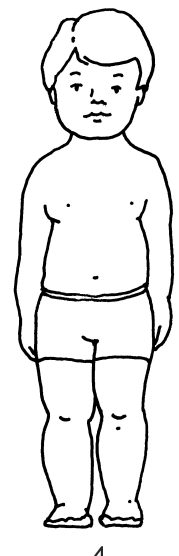

4

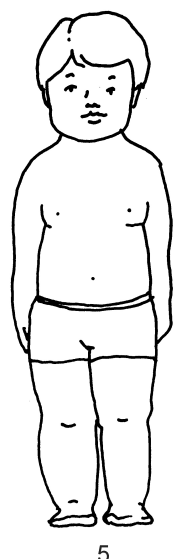

5 
Maternal perception of body size Infant and young child feeding study: WIC

Date:

Participant ID

Questions to ask the mother about her body appearance (Record her response number next to each question):

1. Which of these figures do you think represents a typical Hispanic adult female in your neighborhood?

2. Which of these figures do you think represents a healthy body size for an adult female in your neighborhood?

3. Which of these figures do you prefer to look like?

4. Which of these figures do you think represents your size?

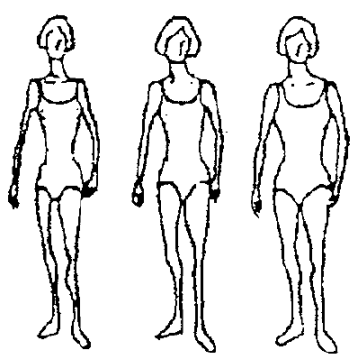

1

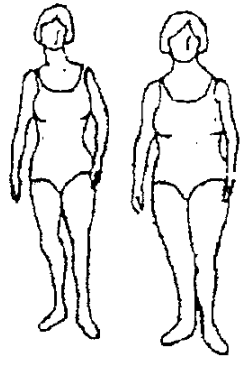

4
5

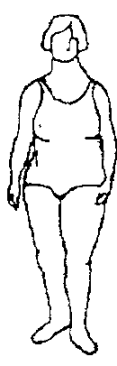

6

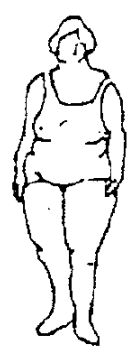

7

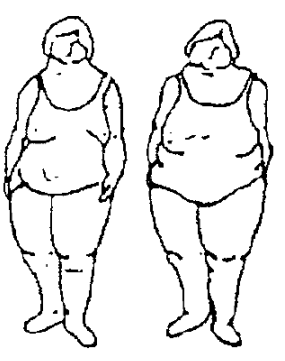

8 


\section{Appendix D: The Parenting Feelings Index}

\section{Parenting Feelings Index (English) Version 6}

Now I am going to read some statements about your feelings about being a mother. I would like you to tell me how often you think, act, or feel in this way. You can respond by marking "Almost Always, Many times, Sometimes, or Rarely." If you think it is not relevant, you can mark Does not Apply.

First name of child

Child code

Date Interviewer

\begin{tabular}{|c|c|c|c|c|c|}
\hline & $\begin{array}{c}\text { Almost } \\
\text { always } \\
\text { (every } \\
\text { day) }\end{array}$ & $\begin{array}{c}\text { Many } \\
\text { times } \\
(2-3 \\
\text { times a } \\
\text { week })\end{array}$ & $\begin{array}{c}\text { Sometimes } \\
\text { (about } \\
\text { once a } \\
\text { week) }\end{array}$ & $\begin{array}{c}\text { Rarely } \\
\text { (did not } \\
\text { happen } \\
\text { last } \\
\text { week) }\end{array}$ & $\begin{array}{c}\text { Does } \\
\text { not } \\
\text { apply }\end{array}$ \\
\hline $\begin{array}{l}\text { 1. I often have the feeling that I cannot handle } \\
\text { things very well. }\end{array}$ & 4 & 3 & 2 & 1 & 0 \\
\hline $\begin{array}{l}\text { 2. I find myself giving up more of my life to } \\
\text { meet my children's needs than I ever } \\
\text { expected. }\end{array}$ & 4 & 3 & 2 & 1 & 0 \\
\hline $\begin{array}{l}\text { 3. I feel trapped by my responsibilities as a } \\
\text { parent. }\end{array}$ & 4 & 3 & 2 & 1 & 0 \\
\hline $\begin{array}{l}\text { 4. Since having this child I have been unable } \\
\text { to do new and different things. }\end{array}$ & 4 & 3 & 2 & 1 & 0 \\
\hline $\begin{array}{l}\text { 5. Since having this child I feel that I am } \\
\text { almost never able to do things that I like to } \\
\text { do. }\end{array}$ & 4 & 3 & 2 & 1 & 0 \\
\hline $\begin{array}{l}\text { 6. I am unhappy with the last purchase of } \\
\text { clothing I made for myself. }\end{array}$ & 4 & 3 & 2 & 1 & 0 \\
\hline $\begin{array}{l}\text { 7. There are quite a few things that bother me } \\
\text { about my life. }\end{array}$ & 4 & 3 & 2 & 1 & 0 \\
\hline $\begin{array}{l}\text { 8. I feel capable and on top of things when I } \\
\text { am caring for my child. }\end{array}$ & 4 & 3 & 2 & 1 & 0 \\
\hline $\begin{array}{l}\text { 9. Having a child has caused more problems } \\
\text { than I expected in my relationship with my } \\
\text { spouse. }\end{array}$ & 4 & 3 & 2 & 1 & 0 \\
\hline 10. I feel alone and without friends. & 4 & 3 & 2 & 1 & 0 \\
\hline $\begin{array}{l}\text { 11. When I go to a party I usually expect not to } \\
\text { enjoy myself. }\end{array}$ & 4 & 3 & 2 & 1 & 0 \\
\hline $\begin{array}{l}\text { 12. I am not interested in people they way I } \\
\text { used to be. }\end{array}$ & 4 & 3 & 2 & 1 & 0 \\
\hline 13. I don't enjoy things the way I used to. & 4 & 3 & 2 & 1 & 0 \\
\hline $\begin{array}{l}\text { 14. My child rarely does things for me that } \\
\text { make me feel good. }\end{array}$ & 4 & 3 & 2 & 1 & 0 \\
\hline $\begin{array}{l}\text { 15. My child will often stay occupied with a } \\
\text { toy for more than } 10 \text { minutes. }\end{array}$ & 4 & 3 & 2 & 1 & 0 \\
\hline $\begin{array}{l}\text { 16. Sometimes I feel that my child doesn't like } \\
\text { me and doesn't want to be close to me. }\end{array}$ & 4 & 3 & 2 & 1 & 0 \\
\hline $\begin{array}{l}\text { 17. My child smiles at me much less than I } \\
\text { expected. }\end{array}$ & 4 & 3 & 2 & 1 & 0 \\
\hline
\end{tabular}




\begin{tabular}{|c|c|c|c|c|c|}
\hline & $\begin{array}{l}\text { Almost } \\
\text { always } \\
\text { (every } \\
\text { day) }\end{array}$ & $\begin{array}{c}\text { Many } \\
\text { times } \\
(2-3 \\
\text { times a } \\
\text { week })\end{array}$ & $\begin{array}{l}\text { Sometimes } \\
\text { (about } \\
\text { once a } \\
\text { week) }\end{array}$ & $\begin{array}{c}\text { Rarely } \\
\text { (did not } \\
\text { happen } \\
\text { last } \\
\text { week) }\end{array}$ & $\begin{array}{c}\text { Does } \\
\text { not } \\
\text { apply }\end{array}$ \\
\hline $\begin{array}{l}\text { 18. When I do things for my child I get the } \\
\text { feeling that my efforts are not appreciated } \\
\text { very much. }\end{array}$ & 4 & 3 & 2 & 1 & 0 \\
\hline $\begin{array}{l}\text { 19. My child knows that I am his or her parent } \\
\text { and wants me more than other people. }\end{array}$ & 4 & 3 & 2 & 1 & 0 \\
\hline $\begin{array}{l}\text { 20. When playing, my child doesn't often } \\
\text { giggle or laugh. }\end{array}$ & 4 & 3 & 2 & 1 & 0 \\
\hline 21. I enjoy being a parent. & 4 & 3 & 2 & 1 & 0 \\
\hline $\begin{array}{l}\text { 22. My child doesn't seem to learn as quickly } \\
\text { as most children. }\end{array}$ & 4 & 3 & 2 & 1 & 0 \\
\hline $\begin{array}{l}\text { 23. My child can be easily distracted from } \\
\text { wanting something. }\end{array}$ & 4 & 3 & 2 & 1 & 0 \\
\hline $\begin{array}{l}\text { 24. My child doesn't seem to smile as much as } \\
\text { most children. }\end{array}$ & 4 & 3 & 2 & 1 & 0 \\
\hline $\begin{array}{l}\text { 25. My child is not able to do as much as I } \\
\text { expected. }\end{array}$ & 4 & 3 & 2 & 1 & 0 \\
\hline $\begin{array}{l}\text { 26. It takes a long time and it is very hard for } \\
\text { my child to get used to new things. }\end{array}$ & 4 & 3 & 2 & 1 & 0 \\
\hline $\begin{array}{l}\text { 27. I feel that I am: (mark one) } \\
\begin{array}{l}\text { 1. Not very good at being a parent } \\
\text { 2. A person who has some trouble } \\
\text { being a parent } \\
\text { 3. Better than average parent } \\
\text { 4. A very good parent }\end{array}\end{array}$ & 4 & 3 & 2 & 1 & 0 \\
\hline $\begin{array}{l}\text { 28. I expected to have closer and warmer } \\
\text { feelings for my child that I do and this } \\
\text { bothers me. }\end{array}$ & 4 & 3 & 2 & 1 & 0 \\
\hline $\begin{array}{l}\text { 29. Sometimes my child does things that bother } \\
\text { me just to be mean. }\end{array}$ & 4 & 3 & 2 & 1 & 0 \\
\hline $\begin{array}{l}\text { 30. My child seems to cry or fuss more often } \\
\text { than other children. }\end{array}$ & 4 & 3 & 2 & 1 & 0 \\
\hline $\begin{array}{l}\text { 31. My child generally wakes up in a bad } \\
\text { mood. }\end{array}$ & 4 & 3 & 2 & 1 & 0 \\
\hline $\begin{array}{l}\text { 32. I feel that my child is very moody and } \\
\text { easily upset. }\end{array}$ & 4 & 3 & 2 & 1 & 0 \\
\hline $\begin{array}{l}\text { 33. My child does a few things which bother } \\
\text { me a great deal. }\end{array}$ & 4 & 3 & 2 & 1 & 0 \\
\hline $\begin{array}{l}\text { 34. When I run into a problem taking care of } \\
\text { my child I have a lot of people to whom I } \\
\text { can talk to get help or advice. }\end{array}$ & 4 & 3 & 2 & 1 & 0 \\
\hline $\begin{array}{l}\text { 35. My child reacts very strongly when } \\
\text { something happens that he/she doesn't like. }\end{array}$ & 4 & 3 & 2 & 1 & 0 \\
\hline $\begin{array}{l}\text { 36. My child gets upset easily over the smallest } \\
\text { thing. }\end{array}$ & 4 & 3 & 2 & 1 & 0 \\
\hline
\end{tabular}




\begin{tabular}{|c|c|c|c|c|c|}
\hline & $\begin{array}{c}\text { Almost } \\
\text { always } \\
\text { (every } \\
\text { day) }\end{array}$ & $\begin{array}{c}\text { Many } \\
\text { times } \\
(2-3 \\
\text { times a } \\
\text { week })\end{array}$ & $\begin{array}{c}\text { Sometimes } \\
\text { (about } \\
\text { once a } \\
\text { week) }\end{array}$ & $\begin{array}{c}\text { Rarely } \\
\text { (did not } \\
\text { happen } \\
\text { last } \\
\text { week) }\end{array}$ & $\begin{array}{c}\text { Does } \\
\text { not } \\
\text { apply }\end{array}$ \\
\hline $\begin{array}{l}\text { 37. My child doesn't seem comfortable when } \\
\text { meeting strangers. }\end{array}$ & 4 & 3 & 2 & 1 & 0 \\
\hline $\begin{array}{l}\text { 38. My child's sleeping or eating schedule was } \\
\text { much harder to establish than I expected. }\end{array}$ & 4 & 3 & 2 & 1 & 0 \\
\hline $\begin{array}{l}\text { 39. I have found that getting my child to do } \\
\text { something or stop doing is: (mark one) } \\
\text { 1. Much harder than I expected } \\
\text { 2. Somewhat harder than I expected } \\
\text { 3. Somewhat easier than I expected } \\
\text { 4. Much easier than I expected }\end{array}$ & 4 & 3 & 2 & 1 & 0 \\
\hline $\begin{array}{l}\text { 40. Think about the number of things that your } \\
\text { child does that bother you - such as } \\
\text { dawdles, refuses to listen, cries, interrupts, } \\
\text { fights, whines, or very active. How many } \\
\text { things did you count? Please mark one. } \\
\text { 1. } 10 \text { or more things bother me } \\
\text { 2. } 7-9 \text { things } \\
\text { 3. } 4-6 \text { things } \\
\text { 4. } 3 \text { or less }\end{array}$ & 4 & 3 & 2 & 1 & 0 \\
\hline $\begin{array}{l}\text { 41. There are some things that my child does } \\
\text { that really bother me a lot. }\end{array}$ & 4 & 3 & 2 & 1 & 0 \\
\hline $\begin{array}{l}\text { 42. My child turned out to be more of a } \\
\text { problem than I expected. }\end{array}$ & 4 & 3 & 2 & 1 & 0 \\
\hline $\begin{array}{l}\text { 43. My child makes more demands of me than } \\
\text { most children. }\end{array}$ & 4 & 3 & 2 & 1 & 0 \\
\hline $\begin{array}{l}\text { 44. I feel that I am successful most of the time } \\
\text { when I try to get my child to do something. }\end{array}$ & 4 & 3 & 2 & 1 & 0 \\
\hline
\end{tabular}




\section{Appendix E: Unadjusted analysis from Aim 1}

Table A-K1 Less healthy food's and healthy food's relationship between acculturation, child age, mother's age, mother's BMI and mother's education status, unadjusted.

\begin{tabular}{|c|c|c|c|c|}
\hline Variable & $\begin{array}{l}\text { Less } \\
\text { healthy } \\
\text { foods } \\
\text { Estimated } \\
\text { coefficient } \\
n=53 \\
\end{array}$ & P-Value & $\begin{array}{l}\text { Healthy } \\
\text { foods } \\
\text { Estimated } \\
\text { coefficient } \\
n=53\end{array}$ & P-Value \\
\hline $\begin{array}{l}\text { Child Age } \\
\text { (months) }\end{array}$ & 0.529 & $0.000^{*}$ & 0.546 & $0.015^{*}$ \\
\hline $\begin{array}{l}\text { Mother Age } \\
\text { (years) }\end{array}$ & 0.131 & 0.467 & 0.159 & 0.615 \\
\hline $\begin{array}{l}\text { Mother BMI } \\
(\mathrm{kg} / \mathrm{m})\end{array}$ & 0.215 & 0.255 & -0.348 & 0.281 \\
\hline $\begin{array}{l}\text { Years } \\
\text { Education }\end{array}$ & -.407 & 0.279 & -1.065 & 0.102 \\
\hline Variable & Difference & P-Value & Difference & P-Value \\
\hline $\begin{array}{l}\text { High } \\
\text { Acculturation } \\
\text { (versus low) }\end{array}$ & -1.701 & 0.503 & -6.874 & 0.073 \\
\hline
\end{tabular}


Table A-K2 Mother's actual versus perceived perception of her self and her child and it's relationship between acculturation, child age, mother's age, mother's BMI and mother's education status: unadjusted.

\begin{tabular}{|c|c|c|c|c|}
\hline Variable & $\begin{array}{l}\text { Mother's } \\
\text { perception of } \\
\text { self } \\
\text { coefficient }\end{array}$ & P-Value & $\begin{array}{l}\text { Mother's } \\
\text { perceptions of } \\
\text { her child } \\
\text { coefficient }\end{array}$ & P-Value \\
\hline Child Age & 0.035 & 0.107 & -0.020 & 0.237 \\
\hline Mother Age & -0.076 & $0.020^{*}$ & -0.049 & $0.050^{*}$ \\
\hline $\begin{array}{l}\text { Education } \\
\text { Status }\end{array}$ & 0.028 & 0.681 & -0.909 & 0.376 \\
\hline Mother BMI & -0.092 & $0.004 *$ & -0.066 & $0.007 *$ \\
\hline Variable & Difference & P-Value & Difference & P-Value \\
\hline Acculturation & 0.812 & 0.078 & 0.575 & 0.095 \\
\hline
\end{tabular}

Table A-k3 Parenting stress score broken up into it's two subscales: parent related and child related and their relationship between acculturation, child age, mother's age, mother's BMI and mother's education status: unadjusted.

\begin{tabular}{lll|ll} 
Variable & $\begin{array}{l}\text { Parent related } \\
\text { score } \\
\text { coefficient }\end{array}$ & P-Value & $\begin{array}{l}\text { Child derived } \\
\text { score } \\
\text { coefficient }\end{array}$ & P-Value \\
\hline Child Age & 0.004 & 0.584 & 0.002 & 0.571 \\
Mother Age & 0.002 & 0.871 & -0.003 & 0.651 \\
$\begin{array}{l}\text { Education } \\
\text { Status }\end{array}$ & -0.0333 & 0.108 & -0.023 & 0.082 \\
Mother BMI & -0.010 & 0.328 & -0.010 & 0.122 \\
\hline Variable & Difference & P-Value & Difference & P-Value \\
\hline Acculturation & -0.039 & 0.771 & -0.086 & 0.241
\end{tabular}

Regression models were fit for all continuous variables (Child Age-Years of education).

$\mathrm{T}$-tests were fit for acculturation versus healthy and unhealthy foods. 


\section{Appendix F: Informed consent for key informant interviews}

INFORMED CONSENT TO PARTICIPATE IN a research project on acculturation and food frequency, maternal stress and maternal perception.

A research project on the effect of levels of acculturation on children's food intake, maternal stress and maternal perceptions of body size is being conducted by Tracie Chavoor, a student, as a part of her thesis research for the Food Science and Nutrition department at Cal Poly, San Luis Obispo. For this study we want to interview WIC and Head Start persons with key knowledge on Mexican American acculturation, nutrition, maternal stress and body image to inform future focus groups with WIC and Head Start mothers.

You are being asked to take part in an individual interview to discuss your views and perceptions on the role of acculturation in foods offered to children, maternal stress and maternal perceptions of body size. Your participation will take approximately one-half to one hour. Notes will be taken during the interview; it will be audio recorded and transcribed later. If additional questions are needed to clarify any of your comments, I may call you at your office to discuss this briefly. Any telephone conversations will be recorded using written notes. Please note that your participation in the interview is voluntary and you are not required to participate. You may discontinue your participation in the interview at any time without penalty.

Your confidentiality will be protected by keeping all information that could identify you to others separate from the rest of the information collected during personal interviews. You will be given an ID code and the key to the codes will be kept separate from the recorded and written interviews.

The possible risks associated with participation in this study are minimal. If you have any concerns regarding the study you may contact Steve Davis, Chair of the Cal Poly Human Subjects Committee, at (805) 756-7383, or Dr. Susan Opava, Dean of Research and Graduate Programs, at (805) 756-1508.

Potential benefits associated with the study include helping identify key questions to ask during WIC and Head Start maternal focus groups which will help increase understanding of the role of Latino acculturation on foods offered to children and maternal perceptions of body size. This information will be shared with WIC and Head Start administrators.

If you have questions regarding this study or would like to be informed of the results when the study is completed, please contact Tracie Chavoor at (818) 456-8835 or by email at tracie.chavoor@gmail.com.

If you agree to voluntarily participate in this research project as described, please indicate your agreement by signing below. You will receive a copy of this form for your reference. Thank you for your participation in this research.

Signature of Volunteer

Date

Signature of Researcher

Date 


\title{
Appendix G: Key informant interview questionnaire
}

\author{
Key Informant Interview
}

Facility:

Date:

Person Interviewing:

1) In your experience have you noticed that mothers with high acculturation offer different foods to their children than mothers with low acculturation?

2) If so, what do you think may be some reasons this might be different between the two groups?

3) For what foods do you notice change?

4) Have you noticed that mothers with a high level of acculturation experience a different level of parenting stress compared to mothers with low acculturation?

5) If so, what do you think may be some reasons this might be different between the two groups? 
6) Do you think parenting stress affects foods offered to the child and/or child's food intake?

7) Have you noticed that mothers with a high level of acculturation have a different perception of what a healthy baby should look like and/or what they desire their baby to look like than mothers with a low acculturation?

8) If so, what do you think may be some reasons this might be different between the two groups?

9) What do you think may help mothers view an overweight child as unhealthy instead of desirable?

10) Have you noticed that mothers with a high level of acculturation have a different desired body weight for themselves compared to mothers with low acculturation?

11)If so, what do you think may be some reasons for this difference?

12)So far, in my research, children of older moms are eating less food (both healthy foods and unhealthy foods) in general than those of younger mothers. Can you think of any reasons this might be happening?

13)What might be a good way to target younger mothers in decreasing the amount of food they are giving to their children?

14)So far, in my research, as children's age increased their mothers were less accurate in how they perceived their weight. They were more likely to guess their child was thinner than they actually were. Do you have any idea of why this might occur?

15)My research also showed that as mother's BMI increased, their ability to accurately perceive their child's weight as well as their own increased. Do you have any ideas of why that is?

16)Lastly, through the data we saw that as mother's acculturation increased her accuracy in how she perceived her own body weight decreased. Do you have any theories on what would cause this? 


\section{Appendix H: Full table of themes and quotes from Key Informant Interviews}

Table A-L1 Themes and quotes from Key Informant Interviews.

\begin{tabular}{|c|c|c|}
\hline The Question & The Theme & Quotes \\
\hline $\begin{array}{l}\text { "In your experience have } \\
\text { you noticed that mothers } \\
\text { with high acculturation offer } \\
\text { different foods to their } \\
\text { children than mothers with } \\
\text { low acculturation?" }\end{array}$ & $\begin{array}{l}\text { Mothers who are more } \\
\text { acculturated will give } \\
\text { their children more } \\
\text { westernized foods } \\
\text { including fast foods, } \\
\text { sports drinks, and sugary } \\
\text { cereals. }\end{array}$ & $\begin{array}{l}\text { "They're buying into more fast food } \\
\text { and highly refined process food, } \\
\text { sugary drinks. And the mothers with } \\
\text { lower acculturation are still } \\
\text { gravitating towards their native } \\
\text { foods." } \\
\text { "“'It would be my opinion that yes, as } \\
\text { families become more acculturated to } \\
\text { the United States they tend to rely less } \\
\text { on the traditional foods and rely more } \\
\text { on convenience items of our country. } \\
\text { " } \\
\text { "I'd guess that as there is more } \\
\text { acculturation, the more Americanized } \\
\text { the diet. And the more they would be } \\
\text { influenced by things on TV like } \\
\text { Gatorade and Capri suns, especially } \\
\text { those things like breakfast cereals." }\end{array}$ \\
\hline $\begin{array}{l}\text { "If so, what do you think } \\
\text { may be some reasons this } \\
\text { might be different between } \\
\text { the two groups?" }\end{array}$ & $\begin{array}{l}\text { Exposure, convenience } \\
\text { and a desire to fit in are } \\
\text { what lead to dietary } \\
\text { acculturation. }\end{array}$ & $\begin{array}{l}\text { "...And the more they would be } \\
\text { influenced by things on TV..." } \\
\text { "So they are going to be exposed to } \\
\text { foods, even starting in head start they } \\
\text { are going to be exposed more to an } \\
\text { American way of diet" } \\
\text { "Just being exposed, exposure for one, } \\
\text { maybe higher income and less of a tie } \\
\text { to their background...trying to fit in } \\
\text { with American society." } \\
\text { "but their children who attend school } \\
\text { and are much more intermixed with } \\
\text { people who have been here for a long } \\
\text { time they are going to want to be like } \\
\text { their peers..." }\end{array}$ \\
\hline
\end{tabular}




\begin{tabular}{|c|c|c|}
\hline $\begin{array}{l}\text { "Have you noticed that mothers } \\
\text { with a high level of } \\
\text { acculturation experience a } \\
\text { different level of parenting }\end{array}$ & $\begin{array}{l}\text { Stress levels are the } \\
\text { same between focus } \\
\text { groups, but for } \\
\text { different reasons. }\end{array}$ & $\begin{array}{l}\text { "I think both have the same stress." } \\
\text { "Not that one is more stressful than the } \\
\text { other but different kinds of stress." }\end{array}$ \\
\hline & $\begin{array}{l}\text { Life is less stressful } \\
\text { in Mexico then in the } \\
\text { U.S. which could } \\
\text { cause high levels of } \\
\text { stress for someone } \\
\text { newly acculturating. }\end{array}$ & $\begin{array}{l}\text { "Again I'm comparing it to Mexico... } \\
\text { Your life is really low key down there. } \\
\text { You might not have a lot of income but } \\
\text { you don't have a lot of expenses..." } \\
\text { "The number of responsibility in terms } \\
\text { of bills and work is harder. First of all } \\
\text { they don't have a lot of access to work } \\
\text { opportunities or upward mobility so } \\
\text { there's this dream of coming to the } \\
\text { north. And then they get here not } \\
\text { realizing there's a whole variety of } \\
\text { responsibilities to take into } \\
\text { consideration to have a stable } \\
\text { livelihood." }\end{array}$ \\
\hline $\begin{array}{l}\text { "Do you think parenting stress } \\
\text { affects foods offered to the } \\
\text { child and/or child's food } \\
\text { intake?" }\end{array}$ & $\begin{array}{l}\text { Stress would change } \\
\text { what mothers are } \\
\text { feeding their children. } \\
\text { Convenience foods } \\
\text { increase as stress } \\
\text { increases. }\end{array}$ & $\begin{array}{l}\text { "Convenience is key when you're } \\
\text { stressed." } \\
\text { "I think that for example if parents are } \\
\text { stressed after a long day of work they } \\
\text { may tend to gravitate towards faster } \\
\text { foods..." } \\
\text { "If they are stressed out they don't even } \\
\text { think about what to cook they just feed } \\
\text { them .... whatever is easier for them..." }\end{array}$ \\
\hline $\begin{array}{l}\text { "Have you noticed that mothers } \\
\text { with a high level of } \\
\text { acculturation have a different } \\
\text { perception of what a healthy } \\
\text { baby should look like and/or } \\
\text { what they desire their baby to } \\
\text { look like than mothers with a } \\
\text { low acculturation?" }\end{array}$ & $\begin{array}{l}\text { Hispanics, regardless } \\
\text { of acculturation level, } \\
\text { believe that a chubby } \\
\text { baby is a healthy } \\
\text { baby. }\end{array}$ & $\begin{array}{l}\text { "It's a term of endearment you talk } \\
\text { about. You converse about the childs } \\
\text { fat. Its part of saying how cute the baby } \\
\text { is." } \\
\text { "I think that both groups (of } \\
\text { acculturation) have a perception that a } \\
\text { fat baby is a healthy baby, the fatter the } \\
\text { baby is the healthier they are." } \\
\text { "...Hispanics think that chubby kids are } \\
\text { the healthiest." }\end{array}$ \\
\hline
\end{tabular}




\begin{tabular}{|c|c|c|}
\hline $\begin{array}{l}\text { "Have you noticed that } \\
\text { mothers with a high level of } \\
\text { acculturation have a } \\
\text { different desired body } \\
\text { weight for themselves } \\
\text { compared to mothers with } \\
\text { low acculturation?" }\end{array}$ & $\begin{array}{l}\text { A lot of people were } \\
\text { unsure if acculturation } \\
\text { affects body image or } \\
\text { if it is a reflection of } \\
\text { stress, education and } \\
\text { income. }\end{array}$ & $\begin{array}{l}\text { "I don't know if it's the high or the low } \\
\text { acculturation I think that just in general } \\
\text { Hispanics think that chubby kids are the } \\
\text { healthiest" } \\
\text { "Um. I don't know. Possibly. But I would } \\
\text { again link it to income and stress. If you } \\
\text { are stressed out and don't have enough } \\
\text { money to put food on the table and not } \\
\text { enough money to get by. I think you're } \\
\text { less concerned with your weight because } \\
\text { you're too busy with other things and } \\
\text { that's not the top of your worries" }\end{array}$ \\
\hline & $\begin{array}{l}\text { Mother's are more } \\
\text { concerned with their } \\
\text { weight if they have } \\
\text { had a weight related } \\
\text { health issue. }\end{array}$ & $\begin{array}{l}\text { "Also, if you've had health problems like } \\
\text { diabetes and high blood pressure you're } \\
\text { more tuned in to that... I would say it's } \\
\text { more related to actual health problems..." } \\
\text { "Personally I went through gestational } \\
\text { diabetes with m first child... I knew I had } \\
\text { to do something formally because I want } \\
\text { to avoid it as much as I can for my second } \\
\text { pregnancy." }\end{array}$ \\
\hline & $\begin{array}{l}\text { Many Hispanic } \\
\text { women are probably } \\
\text { unconcerned with } \\
\text { their weight because } \\
\text { they have other } \\
\text { priorities. }\end{array}$ & $\begin{array}{l}\text { "If you are stressed out and don't have } \\
\text { enough money to put food on the table...I } \\
\text { think you're less concerned with your } \\
\text { weight... that's not the top of your } \\
\text { worries." }\end{array}$ \\
\hline & $\begin{array}{l}\text { Women who are less } \\
\text { acculturated are more } \\
\text { concerned with their } \\
\text { post pregnancy } \\
\text { weight. }\end{array}$ & $\begin{array}{l}\text { "I've seen moms who haven't been here } \\
\text { for long and they have kids and they're } \\
\text { like 'oh, I'm fat now..." it's the } \\
\text { pregnancy weight..." } \\
\text { "It's amazed me the number of women } \\
\text { that have come in with body shapers on } \\
\text { under their clothes to make them look } \\
\text { better and they've just had a baby. I think } \\
\text { I've seen it more with women with low } \\
\text { acculturation than with women with high } \\
\text { acculturation." }\end{array}$ \\
\hline
\end{tabular}




\begin{tabular}{|c|c|c|}
\hline $\begin{array}{l}\text { "So far, in my research, children } \\
\text { of older moms are eating less food } \\
\text { (both healthy foods and unhealthy } \\
\text { foods) in general than those of } \\
\text { younger mothers. Can you think of } \\
\text { any reasons this might be } \\
\text { happening?" }\end{array}$ & $\begin{array}{l}\text { As mothers grow } \\
\text { older, their parenting } \\
\text { skills increase. This } \\
\text { may lead to less } \\
\text { parenting with food. }\end{array}$ & $\begin{array}{l}\text { "...older mom's may have better } \\
\text { parenting skills and may be using } \\
\text { food less as a parenting tool than } \\
\text { younger moms" } \\
\text { "I think that older moms... after } \\
\text { years of experience realize they don't } \\
\text { have to feed their } 2^{\text {nd }} \text { or } 3^{\text {rd }} \text { child as } \\
\text { much..." } \\
\text { "I would think its because the mom } \\
\text { (older mother) is more mature... " } \\
\text { "Food is used as a calming thing or } \\
\text { reward or punishment..." } \\
\text { "You know if they want chips and } \\
\text { they're going to throw a tantrum its } \\
\text { easier to give it to them then to } \\
\text { actually set those boundaries and } \\
\text { limits and follow through with what } \\
\text { they say (talking about younger } \\
\text { moms)." }\end{array}$ \\
\hline & $\begin{array}{l}\text { Moms are } \\
\text { overfeeding their } \\
\text { children. }\end{array}$ & $\begin{array}{l}\text { "...parents who were low } \\
\text { acculturation that was a really hard } \\
\text { concept for them where they thought } \\
\text { no, they need to eat they need to eat." } \\
\text { "They want to give them more than } \\
\text { they can eat. They get this obsession, } \\
\text { they see their babies not gaining } \\
\text { weight." }\end{array}$ \\
\hline
\end{tabular}




\begin{tabular}{|l|l|l|}
\hline $\begin{array}{l}\text { "So far, in my research, as } \\
\text { children's age increased their } \\
\text { mothers were less accurate in } \\
\text { how they perceived their } \\
\text { weight. They were more likely } \\
\text { to guess their child was thinner } \\
\text { than they actually were. Do you } \\
\text { have any idea of why this might } \\
\text { occur?" }\end{array}$ & $\begin{array}{l}\text { Mom's perception of a normal } \\
\text { weight may be based on the } \\
\text { children around her. Mom's } \\
\text { with older children may not see } \\
\text { their children as overweight } \\
\text { because when they compare } \\
\text { them to the children around } \\
\text { them, they look normal. }\end{array}$ & $\begin{array}{l}\text { "Maybe if they're comparing } \\
\text { with other kids of their age. I } \\
\text { think there's this increase in } \\
\text { weight so what we perceive } \\
\text { as being skinny and } \\
\text { overweight might not fit what } \\
\text { it is really." }\end{array}$ \\
& & $\begin{array}{l}\text { "I think as children get older } \\
\text { there's a higher percentage of } \\
\text { overweight kids so they see } \\
\text { their child thinner compared } \\
\text { to what they're seeing around } \\
\text { them." }\end{array}$ \\
& & $\begin{array}{l}\text { "Being here longer, it seems } \\
\text { more normal to be } \\
\text { overweight..." }\end{array}$ \\
\hline
\end{tabular}




\section{Appendix I: Research interest explanation form for focus groups}

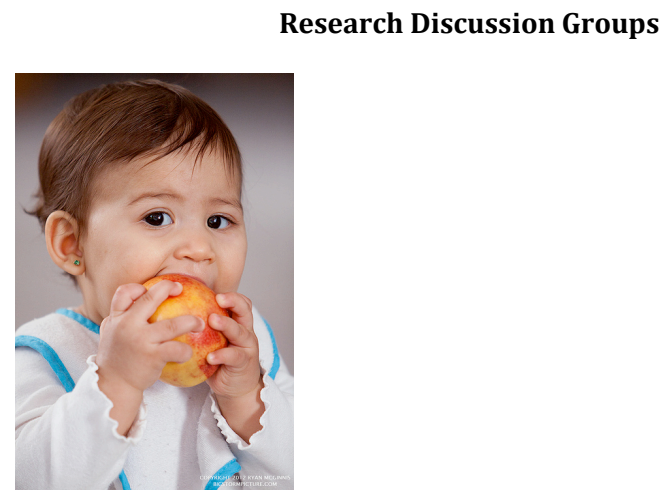

We are looking for mothers who are of Mexican descent who are interested in taking part in a research study! We are trying to see if the diet of children and body image perceptions change when a mother starts to adjust to the culture in the United States.

Requirements: You must be a mother of Mexican descent with a child 3 or younger. The discussion group will last from $1 \frac{1}{2}$ to 2 hours.

What's in it for you? You can help us better understand health and perceptions in your culture so that we can help come up with education materials for mothers like you! You will also receive a $\$ 10$ Wal Mart gift card and there will be snacks and free child care during the discussions.

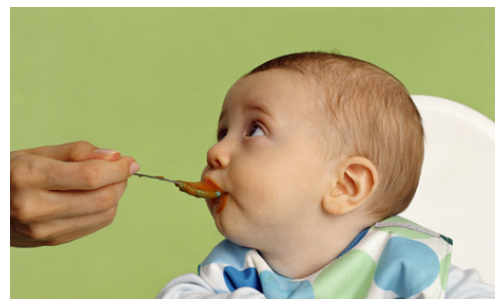




\section{Appendix J: Interest form consent form}

Interest Form For the Research Study: Maternal Acculturation Levels and their effects on children's food intake, maternal stress and maternal perceptions of body size part two: focus groups.

Yes!

I am interested in participating in this study on acculturation

_ I am mother of Mexican decent

I have a child who is 3 years old or younger

Name:

home phone:

cell phone:

work phone:

email:

When is the best time during the day to contact you?

I agree to allow Head Start to release to the key investigator my contact and demographic information above and understand that it will be not shared with any person not involved in the research study. I also understand that this is a Cal Poly study and authorize the researchers to call me to ask me questions about my acculturation and to arrange for participation in a focus group.

(Sign Here)

(Date) 


\section{Appendix K: Focus group informed consent form}

WIC INFORMED CONSENT TO PARTICIPATE IN a research project on acculturation and food frequency, maternal stress and maternal perception.

A research project on the effect of levels of acculturation on children's food intake, maternal stress and maternal perceptions of body size is being conducted by Tracie Chavoor, a student, as part of her thesis research for the Food Science and Nutrition department at Cal Poly, San Luis Obispo.

The purpose of the study:

In this study we want to understand, through small group discussion, the relationship between acculturation in Latina mothers and the food offered to their children. We also would like to know what mothers of this population desire their children to look like and how that affects the foods offered. Ultimately we want to use this information to help design education on foods, health and obesity prevention for Latina WIC mothers. The participants will take part in a group discussion, which will be facilitated by a bilingual member of the community and will last from one and a half to two hours.

Why is this study important?

There are many children who are not being nourished properly and this is causing negative health effects. We want to understand why mothers feed their children the way they do so that we can give them the best education possible on feeding and raising a healthy child.

Benefits and Risks:

The benefit of this study is that it will help us know how to give information to parents in an effective way that will aid them in feeding their children properly.

At the end of the focus group session, to thank you for your time, you will be given a $\$ 10$ gift certificate to Walmart. Snacks and free childcare will also be available on site during the discussion session.

Participation in the focus group may give you a chance to get to know the other mothers in your community better.

There is a minor psychological risk. It is possible that answering some of the questions may make you uncomfortable, talking about your child's weight and how you feed your child. If so, here are ways you can receive support services:

Call 8009444773 for the post-partum depression support line.

Call 8005494499 hotline for transitions mental health.

Contact your primary care physician for referral.

Invitation to Participate:

We would like to invite you to be a part of this study. However, if you do not want to be in this study, it will not affect your benefits from WIC in any way.

If you agree to be in this study, we will call you over the phone to fill out a short questionnaire and to schedule an appointment to spend an hour and a half to two hours in a group of 4 to 7 other mothers to discuss some questions. The questions will be about how you feed your child(ren), how this might be different for someone who is more or less Americanized than yourself, and what you believe a good weight for your child looks like. 
A bilingual woman will lead the discussion with two note takers and an observer also present. You will be asked about your opinions on children and food. The discussion will be audio-recorded too. During the discussion and on the recording, only first names will be used. Your personal information will be kept confidential. Your name and contact information will be kept on a paper separate from the recorded and noted discussion.

The decision to be in this study or not is entirely up to you. If you do not want to participate, you are free to say, "No". If you agree to take part in the study, but later decide that you do not wish to, you can terminate your participation at any point during or before the focus group discussion.

How will we protect your confidentiality?

The information you provide will only be used for research purposes. The recordings of the discussion (both audio and written) will only include first names. The list of the participants with their contact information, made during recruitment, will be kept separately from the recordings. Reports of the study will not give any names or personal details. Once the study is completed, a copy of the findings will be available in this clinic.

Who do I contact if I want to know more, or if I have a problem at any time?

If you want more information about the study before deciding whether or not to participate, or if you participated in the study and have questions please call: Tracie Chavoor (559) 3040731 for assistance in English and Ozzy Alvarado (909) 261-7970 for assistance in Spanish.

If at any time you have concerns about the manner in which the research has been conducted, you may contact Dr. Steve Davis, Chair of the Cal Poly Human subjects Committee, at 756-2754, sdavis@calpoly.edu or Dr. Susan Opava, Dean of Research and Graduate programs, at 756-1508, sopava@calpoly.edu. 
I, agree to participate in the study on acculturation (print name here) and food intake, maternal stress and maternal perception.

I understand that this study involves research.

I have discussed the advantages and disadvantages of participating in the study.

I agree to answer questions about how I feed my child.

I agree to answer questions about how I feel about my child's weight.

I am aware that I am free to leave the study at any time without any consequence. I will still

be able to attend and make full use of all the facilities at WIC as usual. I freely agree to

participate in this study.

I agree to allow WIC to release to the key investigator my contact and demographic

information and understand that it will be not shared with any person not involved in the research study.

Signed:

Date:

Name:

Witness

Signed:

Date:

Name:

Please keep one copy of this form for future reference. The signed copy will be kept with our study materials. 


\section{Appendix L: Bi-dimensional acculturation scale with demographics for focus group}

\section{recruits}

Participant ID

The Bidimensional Acculturation Scale for Hispanics

A1. How often do you speak English?

¿Con qué frecuencia habla usted inglés?

1. Almost Always/Casi Siempre

2. Often/Frecuentemente

3. Sometimes/Algunas Veces

4. Almost Never/Casi Nunca

$N H D$

A2. How often do you speak in English with your friends? ¿Con qué frecuencia habla usted en inglés con sus amigos?

1. Almost Always/Casi Siempre

4

2. Often/Frecuentemente

3. Sometimes/Algunas Veces

4. Almost Never/Casi Nunca

1

NHD

A3. How often do you think in English?

¿Con qué frecuencia piensa usted en ingles?

1. Almost Always/Casi Siempre

2. Often/Frecuentemente

3. Sometimes/Algunas Veces

4. Almost Never/Casi Nunca

4

3

1

NHD

A4. How often do you speak in Spanish? ¿Con qué frecuencia habla usted en español?

1. Almost Always/Casi Siempre 4

2. Often/Frecuentemente

3. Sometimes/Algunas Veces

4. Almost Never/Casi Nunca

A5. How often do you speak in Spanish with your friends? ¿Con qué frecuencia habla usted en español con sus amigos?

1. Almost Always/Casi Siempre

2. Often/Frecuentemente

3. Sometimes/Algunas Veces

4. Almost Never/Casi Nunca

4

3

1

HD 
A6. How often do you think in Spanish?

¿Con qué frecuencia piensa usted español?

1. Almost Always/Casi Siempre

2. Often/Frecuentemente

3. Sometimes/Algunas Veces

4. Almost Never/Casi Nunca

4

3

1

HD

A7. How well do you speak English? ¿Qué tan bien habla usted inglés?

1. Very well/Muy Bien

2. Well/Bien

3. Poorly/No Muy Bien

4. Very Poorly/Muy Mal

4

3

2

NHD

A8. How well do you read in English? ¿Qué tan bien lee usted en inglés?

1. Very well/Muy Bien

2. Well/Bien

3. Poorly/No Muy Bien

4. Very Poorly/Muy Mal

4

3

2

1

NHD

A9. How well do you understand television programs in English? ¿Qué tan bien entiende usted los programas de televisión en inglés?

1. Very well/Muy Bien

2. Well/Bien

3. Poorly/No Muy Bien

4. Very Poorly/ Muy Mal

4

3

2

1

NHD

A10. How well do you understand radio programs in English? ¿Qué tan bien entiende usted los programas de radio en inglés?

1. Very well/Muy Bien

2. Well/Bien

3. Poorly/No Muy Bien

4. Very Poorly/ Muy Mal 
A11. How well do you write in English? ¿Qué tan bien escribe usted en inglés?

1. Very well/Muy Bien

2. Well/Bien

3. Poorly/No Muy Bien

4. Very Poorly/Muy Mal

NHD

A12. How well do you understand music in English? ¿Qué tan bien entiende usted música en inglés?

1. Very well/Muy Bien

2. Well/Bien

3. Poorly/No Muy Bien

4. Very Poorly/Muy Mal

4

3

2

NHD

A13. How well do you speak Spanish? ¿Qué tan bien habla usted español?

1. Very well/Muy Bien

2. Well/Bien

3. Poorly/No Muy Bien

4. Very Poorly/ Muy Mal

$\begin{array}{ll}4 & \\ 3 & H D \\ 2 & \end{array}$

A14. How well do you read in Spanish? ¿Qué tan bien lee usted español?

1. Very well/Muy Bien

2. Well/Bien

3. Poorly/No Muy Bien

4. Very Poorly/ Muy Mal

4

3

2

1

$H D$

A15. How well do you understand television programs in Spanish? ¿Qué tan bien entiende usted los programas de televisión en español?

1. Very well/Muy Bien

2. Well/Bien

3. Poorly/No Muy Bien

4. Very Poorly/ Muy Mal

$\begin{array}{ll}4 & \\ 3 & H D\end{array}$


A16. How well do you understand radio programs in Spanish? ¿Qué tan bien entiende usted los programas de radio en español?

1. Very well/Muy Bien

2. Well/Bien

3. Poorly/No Muy Bien

4. Very Poorly/ Muy Mal

4

2

A17. How well do you write in Spanish? ¿Qué tan bien escribe usted en español?

1. Very well/Muy Bien

2. Well/Bien

3. Poorly/No Muy Bien

4. Very Poorly/ Muy Mal

A18. How well do you understand music in Spanish? ¿Qué tan bien entiende usted música en español?

1. Very well/Muy Bien

2. Well/Bien

3. Poorly/No Muy Bien

4. Very Poorly/ Muy Mal

4

3

2

$H D$

A19. How often do you watch television programs in English? ¿Con qué frecuencia ve usted programas de televisión en ingles?

1. Almost Always/Casi Siempre

2. Often/Frecuentemente

3. Sometimes/Algunas Veces

4. Almost Never/Casi Nunca

4

1

A20. How often do you listen to radio programs in English?

¿Con qué frecuencia escucha usted programas de radio en ingles?

1. Almost Always/Casi Siempre

4

2. Often/Frecuentemente

3. Sometimes/Algunas Veces

4. Almost Never/Casi Nunca 
A21. How often do you listen to music in English?

¿Con qué frecuencia escucha usted música en ingles?

1. Almost Always/Casi Siempre 4

2. Often/Frecuentemente

3. Sometimes/Algunas Veces

4. Almost Never/Casi Nunca

$\begin{array}{ll}4 & \\ 3 & \\ 1 & N H D\end{array}$

A22. How often do you watch television programs in Spanish?

¿Con qué frecuencia ve usted programas de televisión en español?

1. Almost Always/Casi Siempre

4

2. Often/Frecuentemente

3. Sometimes/Algunas Veces

4. Almost Never/Casi Nunca

A23. How often do you listen to radio programs in Spanish?

¿Con qué frecuencia escucha usted programas de radio en español?

1. Almost Always/Casi Siempre

4

2. Often/Frecuentemente

3. Sometimes/Algunas Veces

4. Almost Never/Casi Nunca

3

$H D$

A24. How often do you listen to music in Spanish?

¿Con qué frecuencia escucha usted música en español?

1. Almost Always/Casi Siempre

2. Often/Frecuentemente

3. Sometimes/Algunas Veces

4. Almost Never/Casi Nunca

4

3

$H D$

\begin{tabular}{|c|c|}
\hline $\begin{array}{c}\text { Non Hispanic Domain (NHD) } \\
\text { (High Acculturation) }\end{array}$ & $\begin{array}{c}\text { Hispanic Domains (HD) } \\
\text { (Low Acculturation) }\end{array}$ \\
Total & Total $/ 12=$
\end{tabular}

A score of 2.5 or more indicates high level of adherence to that domain. A score of 2.5 or more in both domains is indicative of biculturalism. 


\section{Demographic Portion}

1. What is your age? / ¿Cual es su edad?
1. $16-19$
2. $20-24$
3. $25-29$
4. $30-34$
5. $35-40$
6. $40+$

2. How many children live in your home? / ¿Cuantos niños viven en su hogar?
2.23 .3
4. $4 \quad 5.5$
$6.6 \quad 7.7$
8. 8
9. 9
10. $10+$

3. What is your marital status / Cual es su Estado civil:
1. Single/Soltero
2. Married/Casado
3. Cohabitating/Concubinato
4. Widowed/Viudo
5. Divorced/Divorciado

4. What is your work status/Ocupación actual:

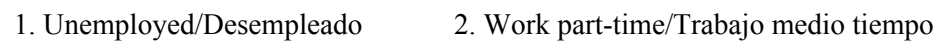

3. Work full-time/Trabajo tiempo completo 4. Student/Estudiante

1. What is your country of origin? Cual es su pais de origen?
1. U.S./ Estados Unidos
2. Mexico/ México 


\section{Appendix M: Demographics Questionnaire for focus group participants}

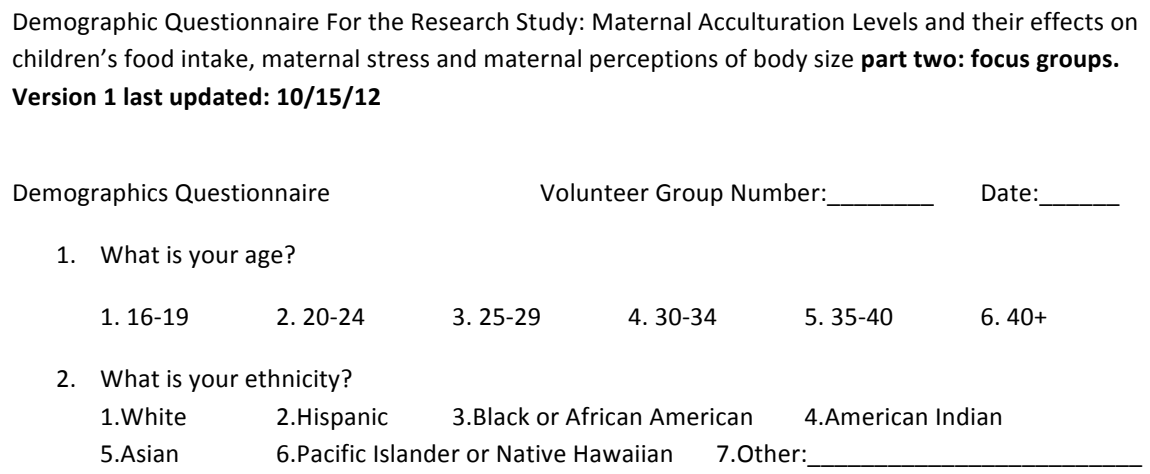

3. What is the age of your youngest child in months?

4. How many children live in your home?
1. 1 2. $2 \quad 3.3$
4.45
7. 78.8
9. 9 10. $10+$

56.6

5. How many people currently reside in your household, adults and children (including yourself)?
1. 1-2
2. 3-4
3. 5-6
4. 7-8
5. 9-10 6. $11+$

6. What is your marital status:
1. Single
2. Married
3. Cohabitating
4. Widowed
5. Divorced

7. What is your work status:
1. Unemployed
2. Work part-time
3. Work full-time
4. Student

8. Education
1. No Schooling Completed
2. Preschool to $8^{\text {th }}$ grade
3. Some High School
4. High School Degree
5. Some College
6. Associate's Degree 7. Bachelor's Degree or more

9. House Hold Income
1. Less than $\$ 10,000$
2. $\$ 10,000-\$ 19,999$
3. $\$ 19,999-\$ 29,999$
4. $\$ 30,000-\$ 39,999$
5. $\$ 40,000-\$ 49,999$
6. $\$ 50,000-\$ 59,999$
7. $\$ 60,000-\$ 69,000$ 


\title{
Appendix N: Focus group questions
}

\author{
Focus Group Questions \\ 1.Tell us your first name and what was your favorite food that your mother made for you \\ when you were a child (or grandmother or whoever did the cooking in your household).
}

2. So now I'd like to learn about how you think the way your mother fed you differs from the way you now feed your child?

3. Do you think that Hispanic mothers who are more Americanized offer different foods to their children compared to someone who is less Americanized?

4. What do you think is most important to Hispanic mothers when they choose what their child is going to eat?

5. Do you think that these factors are different between a mother who is more Americanized than a mother who is less Americanized?

6. Do you think that what a child eats affects his or her weight? And how so? 


\section{Appendix O: Full table of focus group themes}

Focus group themes separated by level of acculturation

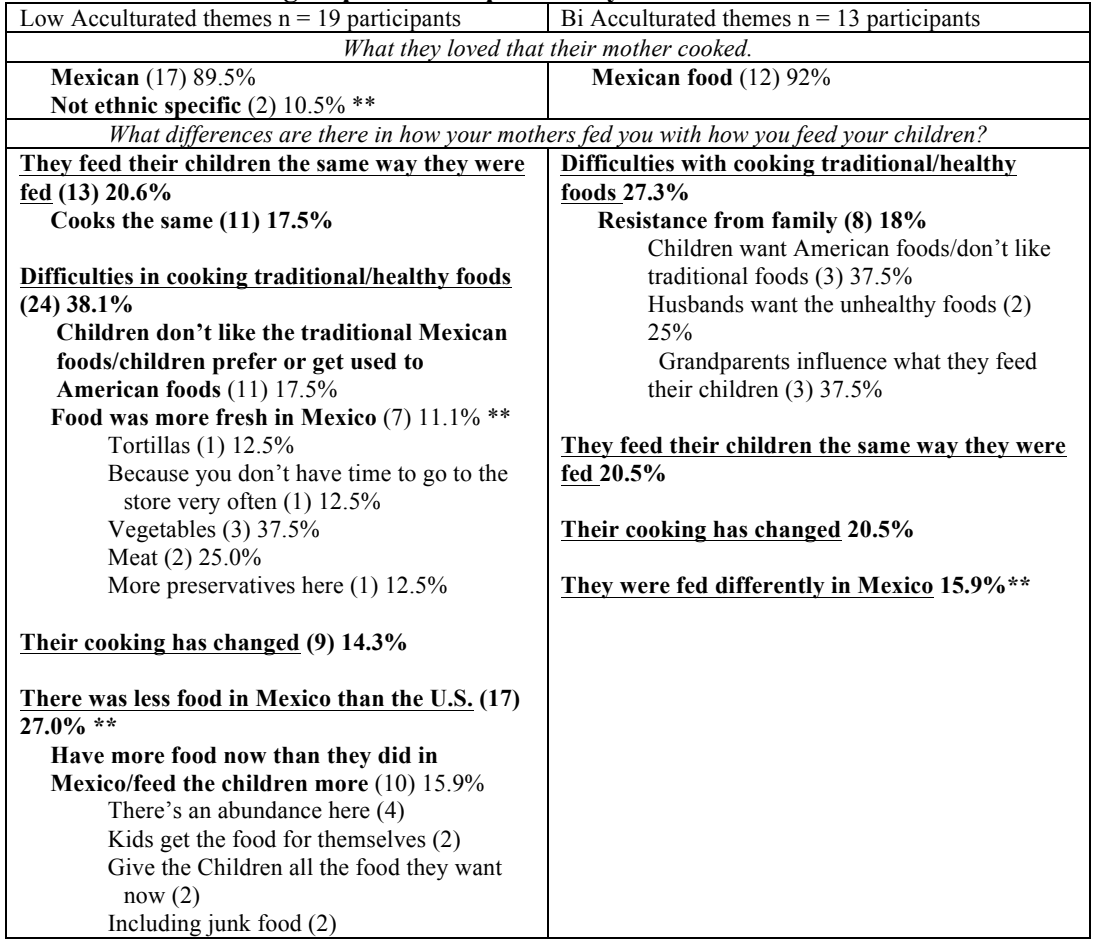




\begin{tabular}{|c|c|}
\hline \multicolumn{2}{|c|}{$\begin{array}{c}\text { So do you think that moms that are more Americanized feed their children differently compared to Latina } \\
\text { moms that are less Americanized? }\end{array}$} \\
\hline Negative affects of acculturation on diet (19) & Negative affects of Americanization on diet \\
\hline $41.3 \%$ & $35.5 \%$ \\
\hline Eat foods that are faster to cook/ more fast & More Americanized cook/eat faster/more \\
\hline foods $(6) 13.0 \%$ & processed foods. $22.6 \%$ \\
\hline Canned (3) & Canned foods (1) $25.0 \%$ \\
\hline Preprepared foods (1) & Cup o noodles (1) $25.0 \%$ \\
\hline Pizza (1) & Frozen Foods (2) 50.0\% \\
\hline Hamburgers (2) & \\
\hline Soups (1) & Age's affect on acculturation/diet $19.4 \% * *$ \\
\hline Chinese food (1) & People who come to the U.S. older are less \\
\hline Frozen Food (2) & likely to change the way they cook $12.9 \%$ \\
\hline Chicken nuggets (1) & The younger generation will make the \\
\hline But still healthy (1) & $\begin{array}{l}\text { healthy changes when they come to the } \\
\text { U.S. } 50.0 \%\end{array}$ \\
\hline & The younger generation realized the health \\
\hline Positive affects of acculturation on diet (16) & affects of diet (1) $50.0 \%$ \\
\hline $34.8 \% * *$ & \\
\hline & Cooking from scratch (5) $16.1 \% * *$ \\
\hline Patterns of less Americanized mothers (5) $10.9 \%$ & There is no difference between less and more \\
\hline$* *$ & Americanized mothers (4) $12.9 \% * *$ \\
\hline & It depends on the mom (2) $66.7 \%$ \\
\hline & $\begin{array}{l}\text { All moms want to give what is best to } \\
\text { their kids (1) } 33.3 \%\end{array}$ \\
\hline
\end{tabular}




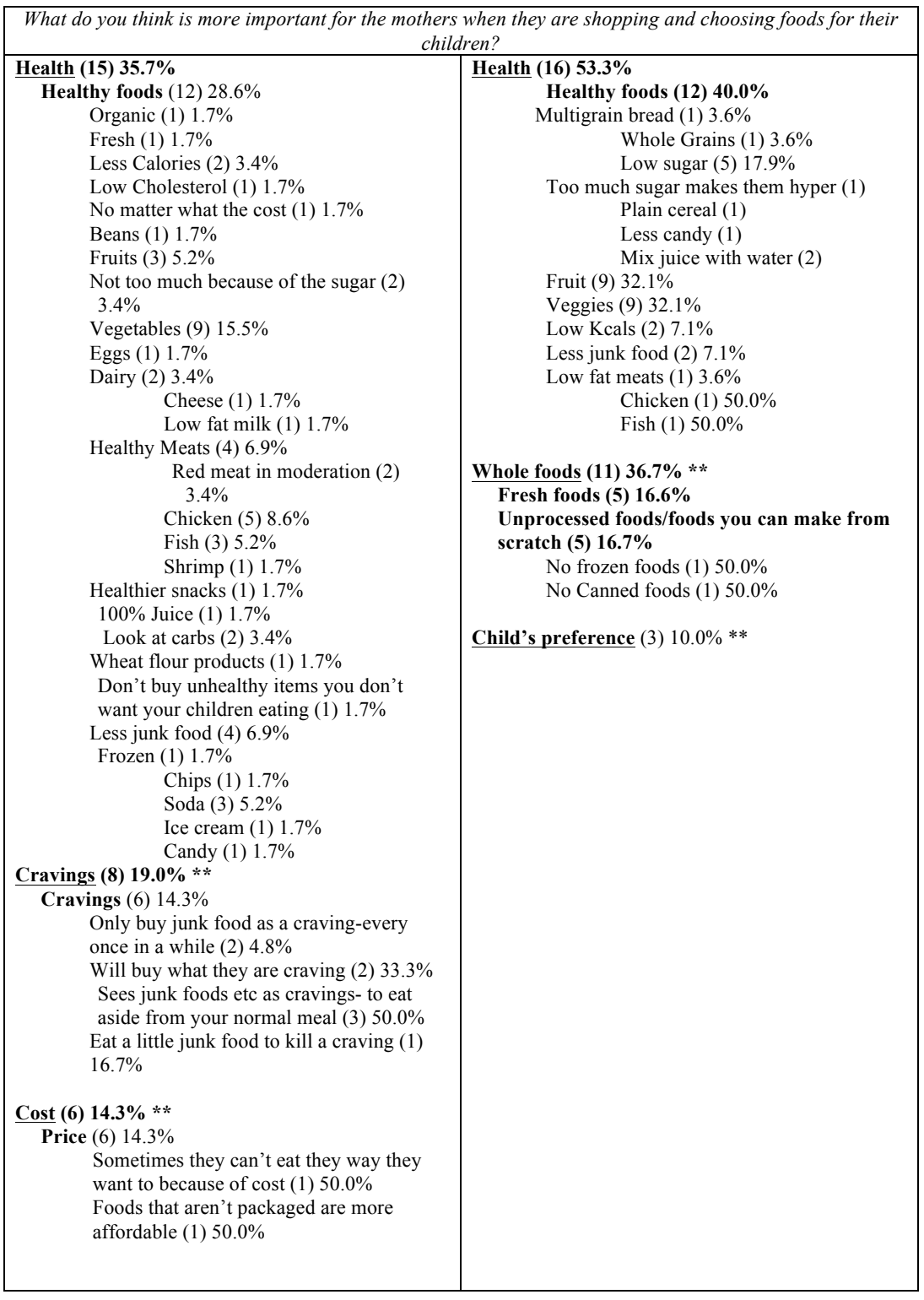




\begin{tabular}{|c|c|}
\hline $\begin{array}{r}\text { Do you think that these factors are different for a mo } \\
\text { Ameri }\end{array}$ & $\begin{array}{l}\text { or that is more Americanized than for one that is less } \\
\text { nized? }\end{array}$ \\
\hline$\frac{\text { Differences for more Americanized (8) } \mathbf{5 0 . 0} \%}{\text { Buy convenience foods (4) } 25.0 \% * *}$ & Differences for more Americanized 21.4\% \\
\hline Differences for less Americanized (2) $12.5 \%$ & $\begin{array}{l}\text { Differences for less Americanized } 35.7 \% \\
\text { Don't realize the effects of a poor diet } \\
14.3 \% * *\end{array}$ \\
\hline $\begin{array}{l}\text { Do not believe there is a difference between more } \\
\text { and less acculturated mothers (4) } 25.0 \%\end{array}$ & Not as active $14.3 \% * *$ \\
\hline $\begin{array}{l}\text { Some care about health and some do not } \\
\text { (4) } 25.0 \% * *\end{array}$ & No, there is no difference $35.7 \%$ \\
\hline Do you think that what a child eat & affects their weight and if so how? \\
\hline Yes (5) $13.5 \%$ & $\underline{\text { Yes }}(8) 20.0 \%$ \\
\hline Some specific foods contribute to weight gain & Specific foods that contribute to weight gain (20) \\
\hline (10) $27 \%$ & $\begin{array}{l}\mathbf{5 0 . 0} \% \\
\quad \text { Junk food/Fast food (10) } 25.0 \% * *\end{array}$ \\
\hline Food is not the main factor in weight (7) $18.9 \%$ & Pizza (2) $28.6 \%$ \\
\hline$* *$ & Hamburger (1) $14.3 \%$ \\
\hline Genetics might matter more (5) $13.5 \% * *$ & French Fries (2) $28.6 \%$ \\
\hline Has two children, feeds them the same & Corn Dogs (1) $14.3 \%$ \\
\hline and one is bigger than the other (1) & Hot dogs (1) $14.3 \%$ \\
\hline $33.3 \%$ & Sweets (4) $10.0 \% * *$ \\
\hline $\begin{array}{l}\text { Family eats well/ exercises but still } \\
\text { overweight (1) } 33.3 \%\end{array}$ & $\begin{array}{l}\text { Soda (1) } 12.5 \% \\
\text { Candy (1) } 12.5 \%\end{array}$ \\
\hline Had a child who grew into their weight (1) & Juice (1) $12.5 \%$ \\
\hline $33.3 \%$ & Chocolate milk (1) $12.5 \%$ \\
\hline & Cookies (3) $37.5 \%$ \\
\hline & Cinnamon rolls (1) $12.5 \%$ \\
\hline Other food related factors that contribute to & \\
\hline weight gain (4) $10.8 \% * *$ & Protectors against weight gain (5) $12.5 \% * *$ \\
\hline & Homemade food is healthier (4) $10.0 \% * *$ \\
\hline
\end{tabular}




\begin{tabular}{|c|c|}
\hline \multicolumn{2}{|c|}{ How do you know if your child is at the proper weight? } \\
\hline $\begin{array}{l}\text { Health care professionals (12) } 48.0 \% \\
\text { WIC (3) } 12.0 \% \\
\text { Doctor (9) } 36.0 \% \\
\text { Didn't listen to the doctor's advice to } \\
\text { feed her underweight child more (1) } \\
10.0 \% \\
\text { Doctor told them to not force food, their } \\
\text { child will eat when they are hungry (1) } \\
10.0 \% \\
\text { Health care professional informed the } \\
\text { mom her child was overweight- she } \\
\text { didn't know before (4) } 40.0 \% \\
\text { Thought her child was thin but the } \\
\text { doctor said they were overweight (2) } \\
20.0 \% \\
\text { Didn't believe the health care } \\
\text { professional, believes her child to be too } \\
\text { thin (1) } 10.0 \% \\
\text { She listened to the doctor and got her } \\
\text { child's weight down (1) } 10.0 \% \\
\text { Parent's using the child's appearance to assess } \\
\text { their child's weight (4) 16.0\% ** } \\
\text { How their clothes are fitting (2) } 8.0 \% \\
\text { Look at their cheeks (1) } 4.0 \% \\
\text { Compare their children to other children } \\
\text { (1) } 4.0 \% \\
\text { Parents' misunderstanding of weight (6) } 24.0 \% \\
\text { Parents don't know how much their child } \\
\text { should weigh (2) } 8.0 \% \\
\text { Parents tend not to notice weight as much } \\
\text { (4) } 16.0 \%\end{array}$ & $\begin{array}{l}\text { Comments about health care professionals (11) } \\
\text { 32.4\% } \\
\text { Doctor (9) } 26.5 \% \\
\text { Wic (1) } 2.9 \% \\
\text { Sometimes doesn't believe the health care } \\
\text { professional (6) } 17.6 \% * * \\
\text { Think they are thinner than the doctor said } \\
\text { (4) } 57.1 \% \\
\text { Feels like their child is not eating enough } \\
\text { but the doctor says they are fine (1) } 14.2 \% \\
\text { Sometimes the healthcare professional } \\
\text { makes them feel bad and they don't want to } \\
\text { confide in them (2) } 28.6 \% \\
\text { Media (5) } 14.7 \% * * \\
\text { Media affects your view (5) } 14.7 \% \\
\text { Their children are seeing thin images in the } \\
\text { media (3) } 50.0 \% \\
\text { Media in Mexico show larger curvier } \\
\text { women (1) } 16.7 \% \\
\text { Media women in mexico more natural (2) } \\
33.3 \%\end{array}$ \\
\hline
\end{tabular}


Do you think there is a difference between more Americanized and less Americanized mothers? Body size differences between more and less Americanized children (9) $42.9 \%$ *

American/Americanized children bigger than Mexican children (3) $14.3 \%$

Maybe they eat differently and that's why (1) $33.3 \%$

Maybe they are born bigger (2) $66.7 \%$ More Americanized children are

smaller/Mexican children larger (6) 28.6\%

Healthier aspects of high acculturated mothers (3) $14.3 \%$ *

Less healthy aspects of low acculturated mothers (4) $19.0 \%$ **

There is no difference between acculturation level and weight expectations (5) $\mathbf{2 3 . 8 \%}$

Every mom is different it doesn't matter how Americanized they are (3) $14.3 \%$

Worrying about weight (9) $\mathbf{5 0 . 0 \%}$ *: Begin to worry about children's weight once they have adapted/Don't worry as much when they first come (7) $38.9 \%$

Because of different priorities (3) $60 \%$

Will worry once they get more education (2) $40 \%$

Beliefs about chubby babies (5) $27.8 \% * *$

These moms think a chubby baby is cute but they know it's not healthy (2) $\mathbf{1 1 . 1 \%}$

Less Americanized think a chubby baby is cute or healthy (3) $16.6 \%$

There is no difference between acculturation and perceptions (4) $22.2 \%$

They do not think differently (4) $22.2 \%$ Everyone is getting the same info from the doctor (1) 


\begin{tabular}{|c|c|}
\hline \multicolumn{2}{|c|}{ Does the way you see your child's weight affect how you feed them? } \\
\hline $\begin{array}{l}\text { Portion Sizes/Amount of foods offered for } \\
\text { overweight kids }(8) \mathbf{1 5 . 7 \%} \\
\text { Try to feed them less/smaller portions ( } 7) \\
13.7 \% \\
\text { Give them portions appropriate for their } \\
\text { age (1) }\end{array}$ & $\begin{array}{l}\text { Portion Sizes/Amount of foods offered for } \\
\frac{\text { overweight kids (5) } \mathbf{1 9 . 2} \%}{\text { Smaller portions/less food (4) } 15.4 \%} \\
\text { Give specific foods to overweight children(4) } \\
15.4 \% * *\end{array}$ \\
\hline $\begin{array}{l}\text { Difficulties in feeding children well (10) } 19.6 \% * * \\
\text { For an overweight child offer more of specific } \\
\text { foods (7) } 13.7 \% * *\end{array}$ & $\begin{array}{l}\text { Physical Activity (4) } 15.4 \% * * \\
\text { Increase physical activity for overweight kids } \\
\text { (4) } \mathbf{1 5 . 4 \%} \\
\text { Non weight related reasons to change diet (5) } \\
\text { 19.2\% ** } \\
\text { Health (5) } \mathbf{1 9 . 2 \%} \\
\text { If their skin looks unhealthy, will feed them } \\
\text { differently (1) } 16.7 \% \\
\text { If the child is unhealthy, feed them } \\
\text { differently (1) } 16.7 \% \\
\text { More veggies(1) } 16.7 \% \\
\text { Child with high cholesterol- give them low } \\
\text { fat, low sugar, high iron (1) } 16.7 \% \\
\text { Iron deficiency: give iron (2) } 33.3 \%\end{array}$ \\
\hline \multicolumn{2}{|c|}{ What you would feed an overweight infant? } \\
\hline $\begin{array}{l}\text { This is difficult (4) } \mathbf{1 2 . 1} \% \\
\text { Decrease the amount of food the infant is } \\
\text { receiving (8) } \mathbf{2 4 . 2 \%} \\
\text { Smaller portions/ less food (8) } 24.2 \% \\
\text { Don't give them too much formula/milk } \\
\text { (3) } 42.9 \% \\
\text { Small portions of meat (1) } 14.3 \% \\
\text { Small portions but more often (1) } 14.3 \% \\
\text { Proper portion sizes (2) } 28.6 \% \\
\text { Offer Specific Foods (11) } 33.3 \% * *\end{array}$ & 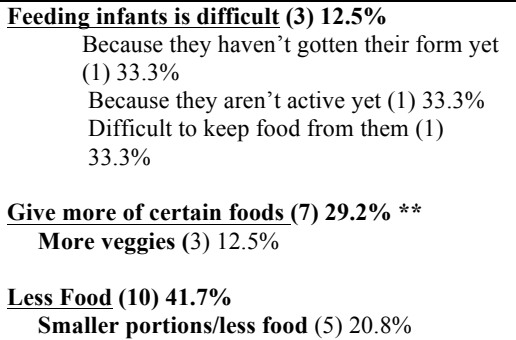 \\
\hline
\end{tabular}




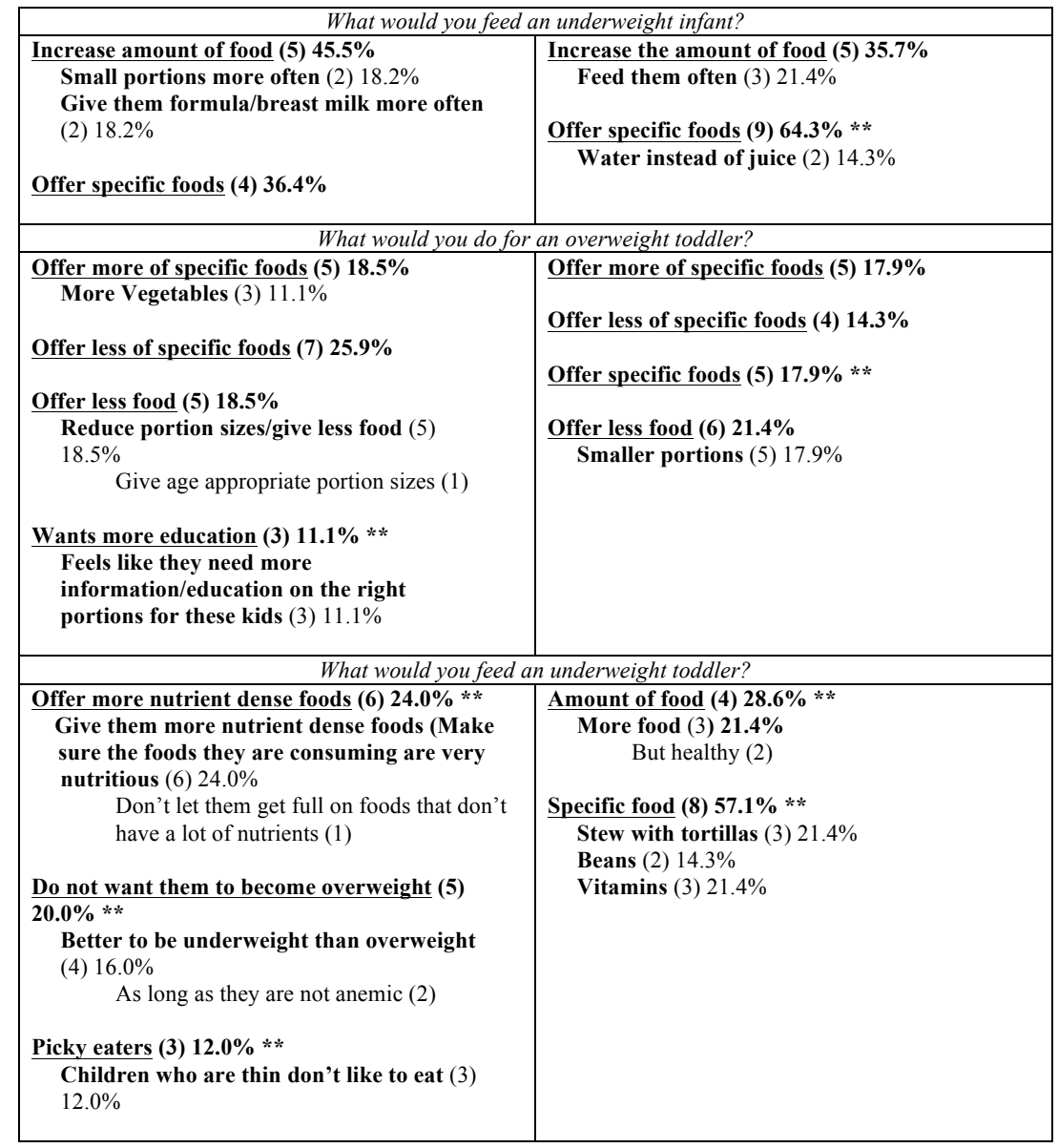




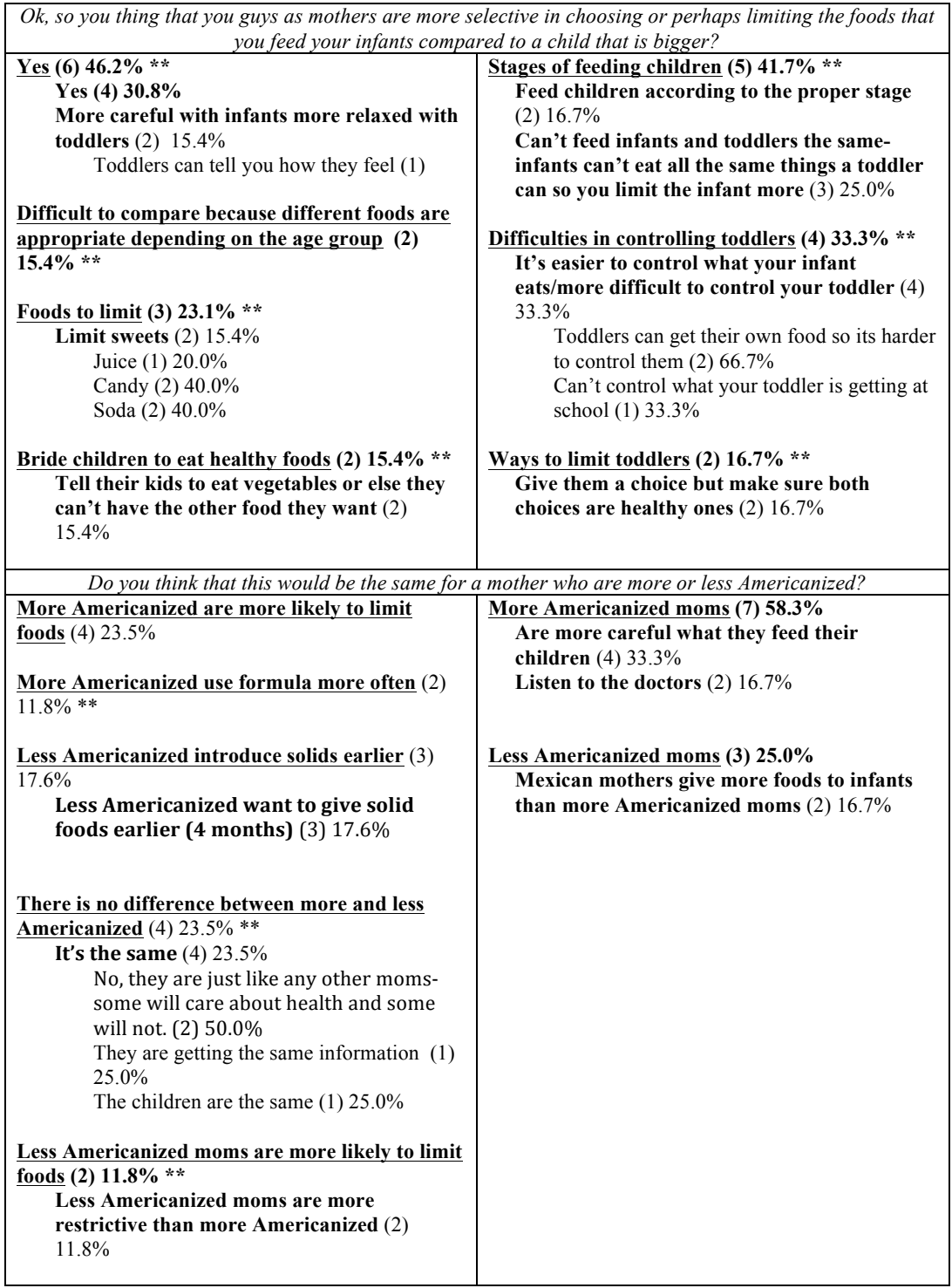

** Refers to a theme that is mentioned in one acculturation group but not the other. 